\title{
GALSIM: The modular galaxy image simulation toolkit
}

Barnaby Rowe a,b,c,*, Mike Jarvis $^{\mathrm{d}, *}$, Rachel Mandelbaum ${ }^{\mathrm{e}, *}$, Gary M. Bernstein $^{\mathrm{d}}$, James Bosch ${ }^{\mathrm{f}}$, Melanie Simet $^{\mathrm{e}}$, Joshua E. Meyers ${ }^{\mathrm{g}}$, Tomasz Kacprzak ${ }^{\mathrm{a}, \mathrm{h}}$, Reiko Nakajima ${ }^{\mathrm{i}}$, Joe Zuntz ${ }^{\mathrm{h}}$, Hironao Miyatake ${ }^{\mathrm{f}, \mathrm{j}}$, Jörg P. Dietrich ${ }^{\mathrm{k}, \mathrm{l}}$, Robert Armstrong ${ }^{\mathrm{f}}$, Peter Melchior $^{\mathrm{m}}$, Mandeep S. S. Gill ${ }^{\mathrm{n}}$

\author{
${ }^{a}$ Department of Physics \& Astronomy, University College London, Gower Street, London, WC1E 6BT, United Kingdom \\ ${ }^{b}$ Jet Propulsion Laboratory, California Institute of Technology, 4800 Oak Grove Drive, Pasadena, CA 91109, United States of America \\ ${ }^{c}$ California Institute of Technology, 1200 East California Boulevard, Pasadena, CA 91106, United States of America \\ ${ }^{d}$ Department of Physics \& Astronomy, University of Pennsylvania, Philadelphia, PA 19104, United States of America \\ ${ }^{e}$ McWilliams Center for Cosmology, Department of Physics, Carnegie Mellon University, 5000 Forbes Ave., Pittsburgh, PA 15213, United States of America

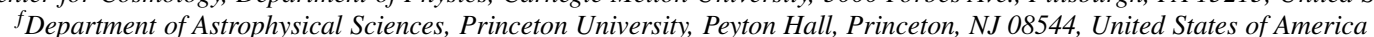 \\ ${ }^{g}$ Kavli Institute for Particle Astrophysics and Cosmology, Department of Physics, Stanford University, Stanford, CA 94305, United States of America \\ ${ }^{h}$ Jodrell Bank Centre for Astrophysics, University of Manchester, Manchester, M13 9PL, United Kingdom \\ ${ }^{i}$ Argelander-Institut für Astronomie, Universität Bonn, Auf dem Hügel 71, D-53121 Bonn, Germany \\ ${ }^{j}$ Kavli Institute for the Physics and Mathematics of the Universe (Kavli IPMU, WPI), The University of Tokyo, Kashiwa, Chiba 277-8582, Japan \\ ${ }^{k}$ Universitäts-Sternwarte München, Scheinerstr. 1, 81679 München, Germany \\ ${ }^{l}$ Excellence Cluster Universe, 85748 Garching b. München, Germany \\ ${ }^{m}$ Center for Cosmology and Astro-Particle Physics and Department of Physics, The Ohio State University, Columbus, OH 43210, United States of America \\ ${ }^{n}$ Kavli Institute for Particle Astrophysics and Cosmology, SLAC National Accelerator Laboratory, Menlo Park, CA 94025-7015, United States of America
}

\begin{abstract}
GALSIM is a collaborative, open-source project aimed at providing an image simulation tool of enduring benefit to the astronomical community. It provides a software library for generating images of astronomical objects such as stars and galaxies in a variety of ways, efficiently handling image transformations and operations such as convolution and rendering at high precision. We describe the GALSIM software and its capabilities, including necessary theoretical background. We demonstrate that the performance of GALSIM meets the stringent requirements of high precision image analysis applications such as weak gravitational lensing, for current datasets and for the Stage IV dark energy surveys of the Large Synoptic Survey Telescope, ESA's Euclid mission, and NASA's WFIRST-AFTA mission. The GALSIM project repository is public and includes the full code history, all open and closed issues, installation instructions, documentation, and wiki pages (including a Frequently Asked Questions section). The GALSIM repository can be found at https://github.com/GalSim-developers/GalSim.
\end{abstract}

Keywords: methods: data analysis, techniques: image processing, gravitational lensing, cosmology: observations

\section{Introduction}

Rapid advances in instrumentation and telescope technology are forcing changes in the techniques used to analyse astronomical data. As data volumes increase, and statistical uncertainties decrease correspondingly, systematic biases resulting from imperfect or approximate inference must be reduced to ensure full return on investments made into increasingly large projects.

An area of research where technology and data volumes are placing increasingly stringent requirements on data analysis methodology is observational cosmology. Recent years have seen a number of wide area, long exposure imaging surveys of the extragalactic sky from both ground-based telescopes (e.g. $\operatorname{SDSS}^{1}$, CFHTLS ${ }^{2,3}$ : see Abazajian et al., 2009;

\footnotetext{
${ }^{*}$ Corresponding author

Email addresses: browedstar.ucl.ac.uk (Barnaby Rowe), michael@jarvis. net (Mike Jarvis), rmandelb@andrew. cmu . edu (Rachel Mandelbaum)

${ }^{1}$ http: //www.sds. org/

2 http://www. cfht.hawaii.edu/Science/CFHTLS/

${ }^{3}$ http://www.cfhtlens.org/
}

Heymans et al., 2012, respectively) and space $\left(\operatorname{COSMOS}^{4}\right.$ : see Scoville et al., 2007). Data from these projects continue to be exploited for their rich scientific content.

More ambitious ground-based projects are already underway, including the Dark Energy Survey ${ }^{5}$ (DES: see e.g. Sánchez et al., 2010), Hyper Suprime-Cam ${ }^{6}$ (HSC: see Miyazaki et al., 2012) and the Kilo Degree Survey ${ }^{7}$ (KiDS: see de Jong et al., 2013). By most measures these upcoming surveys of the deep extragalactic sky will bring an order of magnitude more imaging data than their recent predecessors. In the 2020s, the ground-based Large Synoptic Survey Telescope $\left(\mathrm{LSST}^{8}\right)$, and the ESA Euclid ${ }^{9}$ and NASA WFIRST-AFTA ${ }^{10}$ space missions, will be taking extragalactic imaging data in

\footnotetext{
${ }^{4}$ http://cosmos.astro.caltech.edu/

5 http://www. darkenergysurvey.org/

${ }^{6}$ http://www.naoj.org/Projects/HSC/

${ }^{7}$ http://kids.strw.leidenuniv.nl/

${ }^{8}$ http: //www.lsst.org/lsst/

${ }^{9}$ http://sci.esa.int/euclid/, http://www.euclid-ec. org

${ }^{10}$ http: //wfirst.gsfc.nasa.gov/
} 
vast quantities. These successive generations of projects are examples of "Stage III" and "Stage IV" dark energy surveys (Albrecht et al., 2006).

The increasing data volumes in these planned surveys are due to increases in survey area and, to a somewhat lesser extent, depth, as motivated by their science goals (Albrecht et al., 2006; Peacock et al., 2006). Increasing area and depth brings a greater number of galaxy objects, and thus a decrease in the statistical uncertainties on final measurements. However, this means that the tolerable systematic error in the inferred properties of each galaxy needs to be reduced commensurately. As surveys become larger, the accurate estimation of galaxy properties from noisy images becomes increasingly important. In this context the GALSIM project was conceived, aiming to provide a common image simulation tool for use across multiple surveys and to aid comparison between measurement methods.

Weak gravitational lensing (for reviews see, e.g., Schneider, 2006; Bartelmann, 2010; Huterer, 2010) is a prime example of a scientific application that relies on reliable inference regarding the properties of astrophysical objects from imperfect images. Here the shape of the galaxy (typically some property related to its ellipticity) is used to construct a noisy estimate of gravitational shear, which can be related to second derivatives of the projected gravitational potential and is thus sensitive to all matter (including dark matter). Weak lensing can be used to constrain both cosmic expansion and the growth of matter structure over time, and is thus valuable as a test of dark energy and modified gravity models (e.g. Albrecht et al., 2006; Peacock et al., 2006).

To achieve this potential as a probe of cosmology, however, the weak lensing community must control additive and multiplicative systematic biases in shear estimates to $\sim 2 \times 10^{-4}$ and $2 \times 10^{-3}$, respectively (e.g. Huterer et al., 2006; Amara \& Réfrégier, 2008; Massey et al., 2013; Mandelbaum et al., 2014). This accuracy must be achieved in the presence of detector imperfections, telescope blurring and distortion, atmospheric effects (for ground-based surveys), and noise (galaxies used for lensing typically have a signal-to-noise ratio of the flux as low as 10). Weak lensing inference for cosmology presents a significant technical challenge.

Simulations of astronomical imaging data will play an important part in meeting this challenge. For example, weak lensing estimators typically invoke non-linear combinations of image pixels, and can be shown to suffer from generic systematic biases when applied to noisy galaxy images (e.g. Kaiser, 2000; Bernstein \& Jarvis, 2002; Hirata et al., 2004; Refregier et al., 2012; Melchior \& Viola, 2012) unless constructed extremely carefully (Kaiser, 2000; Bernstein \& Armstrong, 2014). Simulations of weak lensing survey data in the GREAT08 (GRavitational lEnsing Accuracy Testing 2008) challenge (Bridle et al., 2010) clearly showed the impact of noise in a blind comparison of shear estimation methods. This helped reinvigorate activity aimed at better characterizing these "noise biases" (Refregier et al., 2012; Melchior \& Viola, 2012; Kacprzak et al., 2012) or eliminating them (Bernstein \& Armstrong, 2014).
Another type of bias arises when true galaxy surface brightness profiles do not match the models being fit, often referred to as model bias or underfitting bias (e.g. Voigt \& Bridle, 2010; Bernstein, 2010; Melchior et al., 2010; Kacprzak et al., 2014). One of the goals of GREAT3 (Mandelbaum et al., 2014), the third challenge in the GREAT series, is to explore the impact of model bias across a range of shear measurement methods. For one of its "experiments", it takes real galaxy images from the Hubble Space Telescope (HST) as the underlying surface brightness profiles of the galaxies, and draws sheared versions of these profiles using a method derived from that of Mandelbaum et al., 2012: see $\$ 6.5$ in this article. GREAT3 also involved controlled tests of the impact of multiepoch (i.e. multiple exposure) imaging and realistic uncertainty about the point spread function (PSF) in survey data (Mandelbaum et al., 2014). The initial imperative for developing GALSIM was specifically to enable the creation of the required images for the GREAT3 challenge.

But there are other issues in both space and groundbased data that are still to be fully addressed for precision photometry and shape estimation. These include object confusion (or deblending), PSF-object colour mismatching (Cypriano et al., 2010), differential atmospheric chromatic refraction (Meyers \& Burchat, 2014), galaxy colour gradients (Voigt et al., 2012; Semboloni et al., 2013), and non-linear detector effects (e.g. Rhodes et al., 2010; Seshadri et al., 2013; Antilogus et al., 2014). Simulation investigations into the many different aspects of the shear measurement problem will continue to improve our understanding of how to meet these challenges for upcoming surveys.

Systematic biases are likely to be present, to some degree, in all practical estimators of shear, and thus simulations of weak lensing observations will be required for precision cosmology: either to estimate (and thus calibrate) biases for a given survey, or (ideally) to demonstrate that they have been controlled to tolerable levels. The same is also true of the many applications that rely on highly accurate photometry and astrometry. These measurements often require steps such as the classification and removal of outliers, the application of calibration corrections, and the fitting of models to data; simulated tests of these techniques are typically necessary. Those who study galaxy properties by fitting parametric models in order to learn something about galaxy evolution also benefit from controlled simulations with which to test their analysis methods. Motivated by these requirements, the GALSIM project has drawn contributions from members of multiple Stage III and Stage IV survey collaborations, aimed at producing an open-source, community-vetted toolkit for building these indispensable image simulations.

The structure of this paper is as follows. In $\$ 2$ we provide an overview of the GALSIM software toolkit, and describe the motivation and structure of the different component parts of GALSIM. In $\$ 2.3$ we provide references to where these component parts are described, in greater detail, in the rest of the paper.

In $\S 3$ we describe the types of astronomical objects that GALSIM can currently represent. In $\$ 4$ we describe the GALSIM "lensing engine", which generates cosmologically moti- 
vated shear fields. In $\$ 5$ we describe how transformations between coordinate systems are represented, including between image pixel coordinates and "world coordinate systems" (which may use either celestial coordinates or a Euclidean tangent plane approximation). In $\S 6$ we describe how GALSIM objects are rendered to form images, and $\$ 7$ describes the noise models that can be invoked to add noise to images. In $\S 8$ we describe some of the shear estimation routines that come as part of GALSIM.

In $\$ 9$ we describe the numerical validation of GALSIM via series of tests and comparisons, and $\S 10$ discusses performance. In $\$ 11$ we highlight some important effects in real data that GALSIM does not currently include, and we end with a summary and conclusions in $\S 12$.

\section{Software overview}

The design and characteristics of GALSIM arose through the need to meet goals and requirements that were set down early in the life of the project. We describe these, along with some aspects of the collaborative development process, and show how they led to the current state of the GALSIM software. All of the code described in this paper can be found in version 1.2 of GALSIM.

\subsection{Science requirements}

The basic capabilities of GALSIM were driven by the need to meet the following requirements for simulating astronomical images:

1. The ability to flexibly represent and render a broad range of models for astronomical objects in imaging data, including observationally-motivated PSF and galaxy profiles.

2. Handling of coordinate transformations such as shear, dilation and rotation for all objects being rendered, motivated by the need to simulate weak lensing effects at high precision.

3. Representation of the convolution of two or more objects, to describe the convolution of galaxy surface brightness profiles with PSFs.

4. Accuracy in object transformation, convolution and rendering at the level required for Stage IV surveys.

5. Flexibility in specifying image pixel noise models and data configurations (e.g. overlapping objects), so as to allow the importance of these data properties to be evaluated in controlled tests.

6. The ability to do all the above for galaxy or PSF models generated from an input image, e.g. from the HST, so as to be able to compare results against those obtained from simpler parametric prescriptions for galaxies and PSF profiles.

As can be seen, many of these requirements are driven by the need to simulate weak lensing observations. Precision simulation of astrometry and photometry places similar require- ments. ${ }^{11}$ Software which meets the above requirements is therefore of great value for simulations that require accurate photometry (e.g. for photometric redshift estimation).

\subsection{Software design requirements}

In addition to the scientific goals for the GALSIM project, an emphasis was also placed on software design and implementation considerations. GALSIM was conceived to be freely available under an open-source (BSD-style) license, and written in non-proprietary programming languages, making it available to all. This aim is satisfied by the choice of Python in combination with $\mathrm{C}++$.

GALSIM was also designed to be modular, i.e. separated into various functional components, and thus easily extensible. Enforcing modularity allows parts of the code to be written and maintained independently by multiple individuals. This design feature was important in allowing GALSIM to be developed collaboratively. Within a modular program design there is scope for different modules (with a common interface) to be used interchangeably, and this property was crucial in providing the flexibility demanded by GALSIM’s science goals (\$2.1: item 1). Future extensions to the project also occur naturally within this framework as additional modules.

To ensure that the learning curve for GALSIM users is as easy as possible, we require clear documentation on all new features before they are deployed, and major features are demonstrated in heavily-commented example scripts that amount to a kind of tutorial for how to use GalSim. Writing these example scripts has been very useful as a design tool to help determine what user interface is most natural for realistic applications.

Similarly, all new code also requires an accompanying test suite before being merged into the main code base. While we have not tried to impose the precepts of test-driven development within the collaboration, we do take seriously the requirement that all code be tested as comprehensively as possible. In addition to checking the general validity of new code, the extensive unit tests have proved extremely valuable for dealing with issues of cross-platform portability: we regularly run the tests on a wide variety of systems with various idiosyncrasies.

Finally, we require all new code to undergo extensive code review by the rest of the development team, including review of the code, the documentation, and the unit tests. This review process is facilitated by the "Pull Request" feature of GiTHuB ${ }^{12}$, where the code is hosted. The other members of the collaboration team can easily see the set of changes being proposed and even comment on individual lines of code. While bugs are inevitable at some level no matter how much care is taken to avoid them, these steps have been quite effective at catching bugs and documentation errors before code is deployed.

\subsection{The structure of GALSIM}

Given the emphasis on modularity, and the desire to make the software easily useable for as many scientific projects and applications as possible, GALSIM was conceived from the outset

\footnotetext{
${ }^{11}$ For example, see http://www.lsst.org/files/docs/SRD. pdf.

12 https://github.com
} 
to be a toolkit of library routines rather than as a "monolithic" package. From the user's perspective GALSIM is fundamentally a Python class library, providing a number of objects that can be employed for astronomical image simulation in almost any combination specified by the calling code.

In addition, for users who may be more comfortable using configuration files than writing Python code, we also provide a stand-alone executable that reads relatively simple configuration files, which can carry out most of the important GALSIM functionality. In particular, each of the tutorial example scripts have a corresponding configuration file that produces the same output files ${ }^{13}$. Information about the configuration file interface can be found on the GALSIM wiki ${ }^{14}$.

The classes and functions in the GALSIM toolkit can be separated into the following broad categories by functionality. These are (with references to relevant Sections of this article):

- Representing astronomical objects, including any transformations, distortions, and convolutions (see §3).

- Generating gravitational lensing distortions to be applied to astronomical objects (see $\S 4$ ).

- Characterizing the connection between image coordinates and world coordinates (see $\$ 5$ ).

- Rendering the profiles into images (see $§ 6$ ).

- Generating random numbers, and using these to apply noise to images according to physically-motivated models (see $§ 7)$.

- Estimating the shapes of objects once these have been rendered into images (useful for testing, see §8).

For information about the practical use of these tools, we refer the reader to the online documentation available at the GALSIM project page $^{15}$. Further information and a forum for questions regarding the structure or usage of the GALSIM software can be found in the repository Issues pages, or via the galsim tag on the StackOverflow web site ${ }^{16}$.

While Python serves as the principal user interface to GALSIM, many of the numerical calculations are actually implemented in $\mathrm{C}++$. However, this should be considered a mere implementation detail; the structure of GALSIM is intended to prevent the user from needing to interface with the $\mathrm{C}++$ layer directly.

In this paper, we will focus on a scientific, theoretical description of the output generated by GALSIM's classes and functions, and the validation of those outputs to ensure they meet our accuracy requirements.

\footnotetext{
13 Actually, as of version 1.2, the chromatic functionality has yet to be ported over to the configuration interface, so the example script for that (demo12) does not yet have a corresponding configuration file. We plan to add this functionality in the near future.

14 https://github.com/Galsim-developers/Galsim/ wiki/Config-Documentation

${ }^{15}$ See https://github.com/GalSim-developers/Galsim. The example scripts and Quick Reference Guide provide a good starting point for documentation, in addition to the Python docstrings for individual functions and class methods.

${ }^{16}$ http: //stackoverflow.com/tags/galsim/info
}

\section{Surface brightness profiles}

In this section we describe the kinds of surface brightness profiles that are available in GALSIM. We include their analytic formulae where appropriate, and the physical and observational motivations for the models.

\subsection{Galaxy models}

\subsubsection{The exponential disk profile}

First identified in M33 by Patterson (1940), and observed systematically by de Vaucouleurs (1959) as a characteristic component of the light profile in a sample of the brightest nearby galaxies, the exponential disk profile provides a good description of the outer, star-forming regions of spiral galaxies (e.g. Lackner \& Gunn, 2012).

The surface brightness of an exponential disk profile varies as

$$
\begin{aligned}
I(r) & =\frac{F}{2 \pi r_{0}^{2}} \mathrm{e}^{-r / r_{0}} \\
& =\frac{F}{2.23057 r_{\mathrm{e}}^{2}} \mathrm{e}^{-1.67835 r / r_{\mathrm{e}}}
\end{aligned}
$$

where $F$ is the total flux, $r_{0}$ is the scale radius, and $r_{\mathrm{e}}=$ $1.67835 r_{0}$ is the half-light radius, the radius that encloses half of the total flux.

It is represented in GALSIM by the Exponential class, and the size can be specified using either $r_{0}$ or the half-light radius.

\subsubsection{The de Vaucouleurs profile}

This profile (first used by de Vaucouleurs, 1948) is found to give a good fit to the light profiles of both elliptical galaxies and the central bulge regions of galaxies that are still actively star-forming (e.g. Lackner \& Gunn, 2012).

The surface brightness of a de Vaucouleurs profile varies as

$$
\begin{aligned}
I(r) & =\frac{F}{7 ! \cdot 8 \pi r_{0}^{2}} \mathrm{e}^{-\left(r / r_{0}\right)^{1 / 4}} \\
& =\frac{F}{0.010584 r_{\mathrm{e}}^{2}} \mathrm{e}^{-7.66925\left(r / r_{\mathrm{e}}\right)^{1 / 4}},
\end{aligned}
$$

where $F$ is the total flux, $r_{0}$ is the scale radius, and $r_{\mathrm{e}}$ is the half-light radius.

De Vaucouleurs profiles are notorious for having both very cuspy cores and also very broad wings. The cusp occurs around the size of the scale radius $r_{0}$, but because of the broad wings, the half-light radius is several orders of magnitude larger ${ }^{17}$. As such, the second formula is more often used in practice.

It is represented in GALSIM by the DeVaucouleurs class, and the size can be specified using either $r_{0}$ or the half-light radius.

Because de Vaucouleurs profiles have such broad wings, it is sometimes desirable to truncate the profile at some radius, rather than allow it extend to infinity. Thus, GALSIM provides the option of specifying a truncation radius beyond which to have the surface brightness drop to zero.

\footnotetext{
${ }^{17}$ More precisely, $r_{\mathrm{e}} \simeq 3459.485 r_{0}$
} 


\subsubsection{The Sérsic profile}

This profile, developed by Sérsic (1963), is a generalization of both the exponential and de Vaucouleurs profiles. The surface brightness of a Sérsic profile profile varies as

$$
\begin{aligned}
I(r) & =\frac{F}{2 n \pi \Gamma(2 n) r_{0}^{2}} \mathrm{e}^{-\left(r / r_{0}\right)^{1 / n}} \\
& =\frac{F}{a(n) r_{\mathrm{e}}^{2}} \mathrm{e}^{-b(n)\left(r / r_{\mathrm{e}}\right)^{1 / n}},
\end{aligned}
$$

where $F$ is the total flux, $r_{0}$ is the scale radius, $r_{\mathrm{e}}$ is the halflight radius, and $a(n)$ and $b(n)$ are known functions with numerical solutions. Note that the index $n$ need not be an integer. Flux normalization determines $a(n)$. To calculate $b(n)$ in GALSIM we start with the approximation given by Ciotti \& Bertin (1999), then perform a small number of iterations of a numerical, non-linear root solver to increase accuracy.

As with the de Vaucouleurs profile, it is standard practice to use the second formula using the half-light radius, since that size more closely matches the apparent size of the galaxy, particularly for larger $n$ values $(n \gtrsim 2.5)$.

The Sérsic profile is represented in GALSIM by the Sersic class, and the size can be specified using either $r_{0}$ or the half-light radius. The $n$ parameter is allowed to range from $n=0.3$, below which there are serious numerical problems, to $n=6.2$, above which rendering inaccuracy may exceed the requirements set for GALSIM (see §9.1).

As with DeVaucouleurs, it is also possible to specify a truncation radius for the profile, rather than allow it to extend indefinitely.

\subsubsection{Galaxy profiles from direct observations}

Observations made using the HST provide high resolution images of individual galaxies that can be used as direct models of light profiles for simulations (Kaiser, 2000; Mandelbaum et al., 2012). These profiles naturally include realistic morphological variation and irregular galaxies, a contribution of increasing importance to the galaxy population at high redshift.

The details of the implementation of such galaxy profiles are covered in two later Sections: $\$ 3.3 .3$, which describes how objects are represented via two-dimensional lookup tables, and $\S 6.5$, which discusses the specifics of how HST galaxy images and their PSFs are handled by GALSIM. However, it is worth stating here that such a model, based on direct observations of a large sample of individual galaxies, is available in GALSIM. It is represented in GALSIM by the RealGalaxy class.

\subsection{Stellar \& PSF models}

\subsubsection{The Airy \& obscured Airy profile}

The finite circular aperture of a telescope gives rise to a diffraction pattern for the light passing through the aperture, an effect first explained by Airy (1835). The resulting image has a central peak surrounded by a series of rings.
The theoretical intensity distribution for a perfectly circular aperture is given by

$$
\begin{aligned}
I(r) & =\left[\frac{J_{1}(v)}{v}\right]^{2}, \\
v & \equiv \pi r D / \lambda,
\end{aligned}
$$

where $D$ is the diameter of the telescope, $\lambda$ is the wavelength of the light (see, e.g., Born \& Wolf, 1999), and $J_{1}$ is the Bessel function of the first kind of order one.

In many telescopes, the light is additionally diffracted by a central circular obscuration - either a secondary mirror or a prime focus camera. The diffraction pattern in this case is also analytic:

$$
I(r)=\left[\frac{J_{1}(v)-\epsilon J_{1}(\epsilon v)}{v}\right]^{2},
$$

where $\epsilon$ is the fraction of the pupil radius (as a linear dimension, not by area) that is obscured.

It is represented in GALSIM by the Airy class, and the size is specified in terms of $\lambda / D$, which dimensionally gives a size in radians, but which is typically converted to arcsec in practice.

\subsubsection{The Zernike aberration model}

Complex, aberrated wavefronts incident on a circular pupil can often be well approximated by a sum of Zernike polynomials (Zernike, 1934). These functions form an orthogonal set over a circle of unit radius.

In GALSIM the convention of Noll (1976) is adopted, which labels these polynomials by an integer $j$. It is also useful to define the polynomials in terms of $n$ and $m$, which are functions of $j$ satisfying $|m| \leq n$ with $n-|m|$ being even ${ }^{18}$. The integers $n$ and $m$ specify the Zernike polynomials in polar coordinates as

$$
\begin{aligned}
& Z_{\text {even } j}=\sqrt{2(n+1)} R_{n}^{m}(r) \cos (m \theta) \\
& Z_{\text {odd } j}=\sqrt{2(n+1)} R_{n}^{m}(r) \sin (m \theta)
\end{aligned}
$$

for integer $m \neq 0$ and integer $n$, and

$$
Z_{j}=\sqrt{n+1} R_{n}^{0}(r)
$$

for $m=0$, where

$$
R_{n}^{m}(r)=\sum_{s=0}^{(n-m) / 2} r^{(n-2 s)} \frac{(-1)^{s}(n-s) !}{s !\left[\frac{n+m}{2}-s\right] !\left[\frac{n-m}{2}-s\right] !} .
$$

The low order Zernike polynomials map to the low order aberrations commonly found in telescopes, such as defocus $(j=$ $4)$, astigmatism $(j=5,6)$, coma $(j=7,8)$, trefoil $(j=9,10)$ etc. In practice, Zernike polynomials have been found to provide a convenient, and compact, approximate model for telescope aberrations.

Given such a model for the wavefront incident at the pupil, the PSF is also determined at a given image plane location. In

\footnotetext{
18 Specifically, the rule is that the Noll indices $j$ are in order of increasing $n$, and then increasing $|m|$, and negative $m$ values have odd $j$ (Noll, 1976).
} 
the Fraunhofer diffraction limit, the PSF is calculated as the Fourier transform of the autocorrelation of the pupil wavefront.

The resulting profile is represented in GALSIM by the OpticalPSF class. The size is specified in terms of $\lambda / D$, just as for Airy. There are also options for specifying a circular central obscuration as well as rectangular support struts. In GALSIM versions through 1.1, aberrations up to Noll index $j=11$ (spherical aberration) can be included, but there are plans to allow for Zernike terms to arbitrary order.

Because the profile is not analytic in either real or Fourier space, the implementation of this class in GALSIM involves creating an image of the PSF in real space by Discrete Fourier Transform, and then using the InterpolatedImage class to handle the interpolation across this image. Therefore, the techniques that we will describe in $\S 6$ for interpolated images also apply to OpticalPSF.

\subsubsection{The Moffat profile}

Moffat (1969) investigated the hypothesis that stellar PSFs could be modelled as a Gaussian seeing kernel convolved by an obstructed Airy diffraction pattern. He discovered that the Gaussian was not a viable model for the seeing component. Instead, he developed an analytic model for stellar PSFs with broader wings that provides a better fit to observations, a model which now bears his name.

The surface brightness of a Moffat profile varies as

$$
I(r)=\frac{F(\beta-1)}{\pi r_{0}^{2}}\left[1+\left(r / r_{0}\right)^{2}\right]^{-\beta}
$$

where $F$ is the total flux, $r_{0}$ is the scale radius, and $\beta$ typically ranges from 2 to 5 . In the limit $\beta \rightarrow \infty$, the Moffat profile tends towards a Gaussian.

It is represented in GALSIM by the Moffat class, and the size can be specified using $r_{0}$, the full-width at half-maximum (FWHM), or the half-light radius. It is also possible to specify a truncation radius for the Moffat profile, rather than allow it to extend indefinitely.

\subsubsection{The Kolmogorov profile}

In a long ground-based exposure, the PSF is predicted to follow a particular functional form due to the Kolmogorov spectrum of turbulence in the atmosphere. Racine (1996) showed that this prediction was indeed a better fit to the observations of stars in long exposures than a Moffat profile.

The surface brightness of a Kolmogorov profile is defined in Fourier space as

$$
\widetilde{I}(k)=F \mathrm{e}^{-\left(k / k_{0}\right)^{5 / 3}},
$$

where $F$ is the total flux, $k_{0}=A \lambda / r_{0}, A \simeq 2.99$ is a constant given by Fried (1966), and here $r_{0}$ is the Fried parameter (to be distinguished from the use of $r_{0}$ to denote the scale radius in other profiles). Typical values for the Fried parameter are on the order of $10 \mathrm{~cm}$ for most observatories and up to $20 \mathrm{~cm}$ for excellent sites. The values are usually quoted at $\lambda=500 \mathrm{~nm}$, and the Fried parameter $r_{0}$ depends on wavelength as $r_{0} \propto \lambda^{-6 / 5}$.

This profile is represented in GALSIM by the Kolmogorov class, and the size can be specified using $\lambda / r_{0}$, the FWHM, or the half-light radius.

\subsection{Generic models}

\subsubsection{The Gaussian profile}

The Gaussian profile has convenient properties: it is relatively compact and analytic in both real space and Fourier space, and convolutions of Gaussian profiles can themselves be written as a new Gaussian profile. Observational properties of Gaussian profiles, such as their second moments, are also typically analytic.

For this reason the Gaussian profile is often used to represent galaxies and PSFs in simple simulations where speed is valued above realism. And thanks to its analytic properties, it has great value for testing purposes. Furthermore, while a single Gaussian profile is generally a poor approximation to both real PSFs and real galaxies, linear superpositions of Gaussian profiles can be used to approximate realistic profiles with much greater accuracy (e.g. Hogg \& Lang, 2013; Sheldon, 2014).

The surface brightness of a Gaussian profile varies as

$$
I(r)=\frac{F}{2 \pi \sigma^{2}} \mathrm{e}^{-r^{2} / 2 \sigma^{2}},
$$

where $F$ is the total flux and $\sigma$ is the usual Gaussian scale parameter.

It is represented in GALSIM by the Gaussian class, and the size can be specified using $\sigma$, the FWHM, or the half-light radius.

\subsubsection{Shapelet profiles}

Shapelets were developed independently by Bernstein \& Jarvis (2002) and Refregier (2003) as an effective means of characterizing compact surface brightness profiles such as galaxies or stars. The shapelets basis set consists of two-dimensional Gaussian profiles multiplied by polynomials, and they have a number of useful properties. For example, they constitute a complete basis set, so in theory any image can be decomposed into a shapelet vector; however, profiles that are not well matched to the size of the Gaussian will require very high order shapelet terms, such that the decomposition is unfeasible in practice (e.g. Melchior et al., 2010).

For images that are relatively well approximated by a Gaussian, they provide a compact representation of the object, since most of the information in the image is described by low order corrections to the Gaussian, which is what the shapelet decomposition provides.

The Shapelet class in GALSIM follows the notation of Bernstein \& Jarvis (2002), although it is also similar to what has been called "polar shapelets" by Massey \& Refregier (2005). The shapelet expansion is indexed by two numbers, $p$ and $q^{19}$ :

$$
I(r, \theta)=\sum_{p, q \geq 0} b_{p q} \psi_{p q}^{\sigma}(r, \theta)
$$

\footnotetext{
19 The shapelet functions happen to be eigenfunctions of the 2D quantum harmonic oscillator. In that framework, $p$ and $q$ count the number of quanta with positive and negative angular momentum, respectively. $N=p+q$ is the total energy and $m=p-q$ is the net angular momentum.
} 


$$
\begin{aligned}
& \psi_{p q}^{\sigma}(r, \theta)=\frac{(-1)^{q}}{\sqrt{\pi} \sigma^{2}} \sqrt{\frac{q !}{p !}}\left(\frac{r}{\sigma}\right)^{m} e^{\mathrm{i} m \theta} e^{-r^{2} / 2 \sigma^{2}} \\
& \quad \times L_{q}^{(m)}\left(r^{2} / \sigma^{2}\right) \quad(p \geq q) \\
& \psi_{q p}^{\sigma}(r, \theta)=\overline{\psi_{p q}^{\sigma}}(r, \theta) \\
& m \equiv p-q .
\end{aligned}
$$

$L_{q}^{(m)}(x)$ are the Laguerre polynomials, which satisfy the recurrence relation:

$$
\begin{aligned}
L_{0}^{(m)}(x) & =1 \\
L_{1}^{(m)}(x) & =(m+1)-x \\
(q+1) L_{q+1}^{(m)}(x)= & {[(2 q+m+1)-x] L_{q}^{(m)}(x) } \\
& \quad-(q+m) L_{q-1}^{(m)}(x) .
\end{aligned}
$$

The functions may also be indexed by $N=p+q$ and $m=$ $p-q$, which is sometimes more convenient. Both indexing conventions are implemented in GALSIM.

One of the handy features of shapelets is that their Fourier transforms are also shapelets:

$$
\begin{aligned}
\widetilde{\psi}_{p q}^{\sigma}(k, \phi)= & \frac{(-i)^{m}}{\sqrt{\pi}} \sqrt{\frac{q !}{p !}}(k \sigma)^{m} e^{\mathrm{i} m \phi} e^{-k^{2} \sigma^{2} / 2} \\
& \times L_{q}^{(m)}\left(k^{2} \sigma^{2}\right) \quad(p \geq q) .
\end{aligned}
$$

This means convolving shapelets in Fourier space is very efficient.

\subsubsection{Interpolated images}

In some cases, it is useful to be able to take a given image and treat it as a surface brightness profile. This requires defining how to interpolate between the pixel values at which the surface brightness is sampled.

One application for this is the use of direct observations of individual galaxies (e.g., from $H S T$ ) as the models for further simulations, already mentioned in $\S 3.1 .4$. See $\S 6.5$ for more about this possibility.

Another application was also mentioned above in §3.2.2. The general aberrated optical PSF is too complicated to model analytically, so the OpticalPSF class is internally evaluated by interpolation over a finite grid of samples of the surface brightness profile.

The Interpolated Image class converts an arbitrary $n \times n$ image array with elements $I_{i j}$ on pixels of size $s$ into a continuous surface brightness distribution

$$
I(x, y)=\sum_{i, j} I_{i j} \kappa(x / s-i, y / s-j),
$$

where $\kappa$ is a real-space kernel chosen from those listed in Table 1 . Each interpolant uses $K \times K$ input pixel values around the given $(x, y)$ to render a sample at that location. Rendering an $M \times M$ output image hence requires $K^{2} M^{2}$ operations, and the footprint of the InterpolatedImage is extended by $K s / 2$ beyond the original input image.
The delta-function kernel yields infinite (or zero) values in direct rendering and is hence a highly ill-advised choice for an InterpolatedImage that is to be rendered without further convolutions. The sinc kernel has infinite extent and hence uses all $N \times N$ input samples to reconstruct each output sample, leading to $N^{2} M^{2}$ operations in a direct-space rendering. In a high-volume simulation this will also be ill-advised. Approximations to the sinc kernel that provide finite support are often found to give a good compromise. Examples include the kernel that represents cubic and quintic (i.e. 3rd and 5th order) polynomial interpolation, and the Lanczos kernel (see Table 1, also Bernstein \& Gruen, 2014). As will be demonstrated in $\$ 9.2$ and $\$ 9.3$, these higher-order interpolation kernels meet stringent performance requirements for the representation of galaxy images.

\subsection{Transformations}

Many of the profiles we have described so far are circularly symmetric and centred on the coordinate origin. GALSIM can, however, use these profiles to represent (for example) elliptical, off-centred surface brightness profiles. Various transformations can be applied to a GALSIM object representing a surface brightness distribution $I(\mathbf{x})$, to return a new object representing $I^{\prime}(\mathbf{x})$.

The overall amplitude of the profile can be rescaled by some factor simply by using the $\star$ operator. It is also possible to get a rescaled profile with a specified value for the new total flux. Either way, the new profile is $I^{\prime}(\mathbf{x})=c I(\mathbf{x})$ for some constant $c$.

Any one-to-one transformation $T$ of the plane defines an image transformation via

$$
I^{\prime}(\mathbf{x})=I\left[T^{-1}(\mathbf{x})\right]
$$

The GALSIM operation transform implements local linear transformations defined by a transformation matrix $\mathbf{A}$ as

$$
T(\mathbf{x})=\mathbf{A x} .
$$

This can account for any arbitrary distortion, rotation, parity flip or expansion of the coordinate system. The user can specify $\mathbf{A}$ directly; alternatively the GALSIM operations rotate, expand, magnify, shear, and lens implement restricted versions of the transformation that are often convenient in practice.

There is also a command called dilate that combines an expansion of the linear size (i.e. A being a multiple of the identity matrix) with an amplitude scale factor to keep the overall flux of the resulting profile unchanged. This is merely a convenience function, but it is handy, since this operation is fairly common.

Finally, the shift function can translate the entire profile by some amount $\mathbf{x}_{0}$. This corresponds to the transformation

$$
T(\mathbf{x})=\mathbf{x}+\mathbf{x}_{0} .
$$

Together, shift and transform enable any arbitrary affine transformation. 
Table 1: Properties of interpolants

\begin{tabular}{lclc}
\hline Name & Formula & Notes $^{a}$ & No. of points $K$ \\
\hline Delta & $\delta(x)$ & Cannot be rendered in $x$ domain; extreme aliasing $\propto 1$. & 0,1 \\
Nearest & $1, \quad|x|<1 / 2$ & Aliasing $\propto k^{-1}$ & 1 \\
Linear & $1-|x|, \quad|x|<1$ & Aliasing $\propto k^{-2}$ & 2 \\
Cubic & piecewise cubic ${ }^{\mathrm{b}}$ & Common choice for $K$ & 4 \\
Quintic & piecewise quintic ${ }^{\mathrm{b}}$ & Optimized choice for $K_{k}(\mathrm{cf} . \S 6.2 .4)$ & 6 \\
Lanczos & $\operatorname{sinc} x \operatorname{sinc}(x / n), \quad|x|<n$ & Common choice for $K$ with $n=3-5$ & $2 \mathrm{n}$ \\
SincInterpolant & \multicolumn{1}{c}{$\operatorname{sinc} x$} & Perfect band-limited interpolation; slowest & $\infty$ \\
\hline
\end{tabular}

${ }^{b}$ Formulae for Cubic and Quintic interpolants are given in the Appendix to Bernstein \& Gruen (2014).

All of these operations are implemented in GALSIM via a wrapper object that exists independently of, and interfaces with, the original profile. This means the code for implementing these transformations (e.g. the effect in Fourier space, or the deflections to apply for photon shooting, see $§ 6.3$ ) exists in a single place, which helps minimize unnecessary duplication and ensure the reliability of the transformation routines.

For reasons discussed in $\S 6$, GALSIM does not currently handle non-affine transformations acting on a single profile (as required by simulations of flexion such as Velander et al., 2011; Rowe et al., 2013), but see $\$ 5$ for how to handle such coordinate transformation across a full image, using the appropriate locally linear approximation at the location of each object.

\subsection{Compositions}

Two or more individual surface brightness profiles may be added together using the + operator to obtain a profile with $I^{\prime}(\mathbf{x})=I_{1}(\mathbf{x})+I_{2}(\mathbf{x})+\ldots$.

The convolution of two or more objects $I_{1}, I_{2}$ is defined as usual via

$$
I^{\prime}(\mathbf{x})=\left(I_{1} \circ I_{2}\right)(\mathbf{x})=\int I_{1}\left(\mathbf{x}^{\prime}\right) I_{2}\left(\mathbf{x}-\mathbf{x}^{\prime}\right) \mathrm{d}^{2} \mathbf{x}^{\prime} .
$$

This is implemented in GALSIM with the Convolve function, which takes a list of two or more objects to be convolved together.

There are also two special functions that can afford some modest efficiency gains if they apply. AutoConvolve performs a convolution of an object with itself, i.e. with $I_{2}(\mathbf{x})=$ $I_{1}(\mathbf{x})$ in equation (29). Autocorrelate performs a convolution of an object with a $180^{\circ}$ rotation of itself, i.e. with $I_{2}(\mathbf{x})=I_{1}(-\mathbf{x})$.

Finally, GALSIM can implement a deconvolution as well. This lets users solve for the solution $I^{\prime}$ to the equation $I_{1}=$ $I^{\prime} \circ I_{2}$, which in Fourier space is simply

$$
\widetilde{I^{\prime}}(\mathbf{k})=\widetilde{I_{1}}(\mathbf{k}) / \widetilde{I_{2}}(\mathbf{k})
$$

The function Deconvolve implements the inverse of a profile (e.g. $I_{2}$ above) in Fourier space, which is a deconvolution in real space. The returned object is not something that can be rendered directly. It must be convolved by some other profile to produce something renderable ( $I_{1}$ in this example).
One common use case for this is to deconvolve an observed image (represented by an InterpolatedImage object) by its original PSF and pixel response, producing an object that would then be reconvolved by some other PSF corresponding to a different telescope and rendered on an image with a new pixel scale. This is the functionality at the heart of GALSIM's implementation of the reconvolution algorithm described in $\$ 6.5$ and used by the RealGalaxy class (\$3.1.4). As will be discussed in $\S 6.5$, the latter PSF must be band-limited at a lower spatial frequency than the original (deconvolved) PSF to avoid serious artifacts in the final image.

\subsection{Chromatic objects}

Real astronomical stars and galaxies have wavelengthdependent intensity distributions. To model this, it is possible in GALSIM to define objects whose surface brightness is a function not only of position, but also of wavelength. These objects can then be rendered as seen through a particular bandpass (the throughput as a function of wavelength).

The simplest of the wavelength-dependent classes is the Chromatic class, which is constructed from an achromatic profile (i.e. any of the profiles described above) and a Spectral Energy Distribution (SED). The SED defines a wavelengthdependent flux for the object.

Chromatic objects can be transformed, added, and convolved using precisely the same syntax as for achromatic objects. The addition of multiple chromatic objects provides a simple way to creating galaxies with non-trivial wavelength-dependent morphologies. A bulge and a disk can have different SEDs; star forming regions or HII regions can also be added in with their own SEDs.

In addition, the various transformations can take functions of wavelength for their arguments. For example, differential chromatic refraction is implemented by a wavelength-dependent shift, and the effects of chromatic variation in Kolmogorov turbulence can be approximately modelled with a wavelengthdependent dilation. GALSIM provides the helper function ChromaticAtmosphere, which encapsulates both of these effects for an atmospheric PSF.

A more detailed discussion of this recent addition to the GALSim toolkit is reserved for future work, but it provides a valuable resource for simulating a number of important wavelength-dependent effects in cosmology (e.g. 
Cypriano et al., 2010; Voigt et al., 2012; Plazas \& Bernstein, 2012; Semboloni et al., 2013; Meyers \& Burchat, 2014).

\section{Lensing shear and magnification}

A primary purpose of GALSIM is to make simulated images that can be used to test weak gravitational lensing data analysis algorithms, which means that a framework for simulating lensing shear and convergence (see, e.g., Schneider, 2006, for a review) is critical. In the weak gravitational lensing limit, the transformation between unlensed coordinates $\left(x_{u}, y_{u}\right.$; with the origin taken to be at the center of a distant galaxy light source) and the lensed coordinates in which we observe galaxies $\left(x_{l}, y_{l}\right.$; with the origin at the center of the observed image) is linear:

$$
\left(\begin{array}{l}
x_{u} \\
y_{u}
\end{array}\right)=\left(\begin{array}{cc}
1-\gamma_{1}-\kappa & -\gamma_{2} \\
-\gamma_{2} & 1+\gamma_{1}-\kappa
\end{array}\right)\left(\begin{array}{l}
x_{l} \\
y_{l}
\end{array}\right) .
$$

Here we have introduced the two components of lensing shear $\gamma_{1}$ and $\gamma_{2}$, and the lensing convergence $\kappa$. The shear describes the anisotropic stretching of galaxy images in weak lensing. The convergence $\kappa$ describes an isotropic change in apparent object size: areas of the sky for which $\kappa$ is non-zero have apparent changes in area (at fixed surface brightness). Lensing magnification is produced from a combination of the shear and convergence.

It is worth noting that even when $\kappa=0$ the transformation matrix in equation (31) will not have a unit determinant in general. Object area (and thus flux when conserving surface brightness) is therefore not conserved by weak lensing shear, an effect which is not always desired when simulating images. For convenience, GALSIM implements a unit determinant shear transformation that conserves object area, while also implementing the non-area conserving shear defined by the weak lensing transformation of equation (31).

In the simplest use case, GALSIM will take single values for the lensing shear and convergence and apply them to objects. In addition, however, GALSIM is able to generate fields of coherent shear and convergence values corresponding to two physical scenarios described below: cosmological weak lensing fields with some power spectrum, and the weak lensing that arises from a spherical NFW halo (Navarro, Frenk, \& White, 1996).

\subsection{Cosmological lensing fields}

GALSIM provides routines to simulate a Gaussian random field approximation to a cosmological lensing signal, characterized wholly by its power spectrum. In reality, shear and convergence fields show significant non-Gaussianity. The intention, therefore, is not highly cosmologically accurate shear and convergence fields, such as would be suitable for an end-to-end test of cosmological parameter determination. The intention is rather to provide basic functionality for making semi-realistic, spatially varying lensing fields so as to be able to test measurement algorithms that find such regimes challenging in general.

There is nothing to prevent any user from using outputs from a more realistic cosmological ray-tracing simulation (e.g. Becker, 2013) to create an observed galaxy shape catalog that includes a realistic shear and convergence field. If realism is required, this procedure should be followed.

\subsubsection{Basic capabilities}

Any shear field can be projected into orthogonal $E$ - and $B$ mode components, named for their similarity to the electromagnetic vector fields with zero curl and zero divergence, respectively (see, e.g. Schneider, 2006; Mandelbaum et al., 2014). GALSIM is capable of taking input $E$ - and $B$-mode shear power spectra as user supplied functions, and generating random realizations of shear and convergence fields drawn from those power spectra. The basic functionality is carried out by the PowerSpectrum class. Shears are generated on a grid of positions, but GALSIM can interpolate to arbitrary positions within the bounds of the grid using the interpolants discussed in $\$ 6.2$.

Observable quantities such as the shear correlation functions are defined using the vectors connecting pairs of galaxies at separation $\theta$,

$$
\begin{aligned}
\xi_{ \pm}(\theta) & =\xi_{++}(\theta) \pm \xi_{\times \times}(\theta) \\
& =\int_{0}^{\infty} \frac{1}{2 \pi}\left[P_{E}(k) \pm P_{B}(k)\right] J_{0 / 4}(k \theta) k \mathrm{~d} k
\end{aligned}
$$

where $J_{0 / 4}$ denotes the 0th and 4th Bessel function of the first kind, as is appropriate for $\xi_{+}$and $\xi_{-}$, respectively. See Schneider et al. (2002) for more details about relating the correlation functions to the $E$ - and $B$-modes of the flat-sky power spectra $^{20}$.

For cosmological shear correlations $P_{B} \simeq 0$ to a very good approximation, although higher order effects (e.g. source redshift clustering) can introduce physical B-modes (see, e.g., Schneider, 2006). It is useful to include $P_{B}$ when generating shears for other purposes, such as when drawing atmospheric PSF anisotropies according to some power spectrum (which typically requires similar amounts of $E$ and $B$ power). Here $P_{E}(k)$ and $P_{B}(k)$ have dimensions of angle ${ }^{2}$; GALSIM can accept the power spectra in various formats and sets of units.

These definitions of observables rely on continuous Fourier transforms, but in practice we implement the calculations using discrete Fourier Transforms (DFT), and we must be careful about the DFT conventions. We assume we have a grid with length $L$ along one dimension and spacing $d$ between grid points. Given a Fourier-space grid of size consistent with that of the input real-space grid, the minimum and maximum onedimensional wavenumbers $|k|$ on the grid are $k_{\min }=2 \pi / L$ and $k_{\max }=\pi / d$. The lensing engine finds the power $P(k)$ according to the value of $k=\sqrt{k_{1}^{2}+k_{2}^{2}}$ on the grid, draws random amplitudes from a Rayleigh distribution based on $\sqrt{P(k)}$ and random phases from a uniform distribution, and transforms back to our real-space grid to get the real-space shear field, with periodic boundary conditions. The standard definition of the correlation function (Eq. 33) uses the angular frequency, non-unitary definition of the 1D Fourier transform:

$$
\widetilde{f}(k)=\int_{-\infty}^{\infty} f(x) \mathrm{e}^{-\mathrm{i} k x} \mathrm{~d} x \equiv \mathcal{F}\{f(x)\} ;
$$

\footnotetext{
${ }^{20}$ Many standard references regarding lensing power spectra work in terms of the spherical harmonics $\ell$, with the power spectrum denoted $C_{\ell}$. In the flatsky limit we can simply swap $\ell$ with wavenumber $k$ and $C_{\ell}$ with $P(k)$.
} 


$$
f(x)=\frac{1}{2 \pi} \int_{-\infty}^{\infty} \widetilde{f}(k) \mathrm{e}^{\mathrm{i} k x} \mathrm{~d} k \equiv \mathcal{F}^{-1}\{\widetilde{f}(k)\} .
$$

If we trace through the impact of this convention on the discrete, finitely-sampled power spectrum, then our use of the NUMPY ${ }^{21}$ package for Fourier transforms (with the more common unitary definition of the DFT) necessitates various normalization factors related to the input grid configuration. For further details, see Appendix A.

\subsubsection{Implementation details}

Any DFT-based algorithm to generate shears according to a power spectrum is subject to limitations due to the implicit choice of a finite range in wavenumber and the difference between a discrete and continuous Fourier transform. GALSIM includes options to ameliorate these limitations:

- GALSIm can optionally decrease (increase) the minimum (maximum) value of $k$ by internally expanding (contracting) the real-space grid before generating shears. This helps avoid problems with missing shear power on various scales; in the case of cosmological power spectra it is particularly recommended for those who wish to properly reproduce the large-scale shear correlation function (on scales comparable to $\sim 1 / 4$ the total grid extent). There is an important tradeoff implicit in the use of these options: the power spectra that result from using internally enlarged grids is not strictly the same as the input one, and there can be $E$ and $B$ mode leakage as a result of using these options.

- GALSIm has a utility to predict the shear correlation function resulting from the use of a limited $k$ range when generating shears. While the output is not exactly what will be generated in reality since the algorithm does not account for the use of a DFT, it permits users to assess in advance whether their grid choices permit them to roughly reproduce shear correlations on the scales they want.

- A natural consequence of the limited $k$ range is that aliasing can occur if users supply a power spectrum with power below the grid Nyquist scale. GALSIM shear generation routines can optionally band-limit the input power spectrum by applying a hard or soft cutoff at the Nyquist scale to avoid aliasing.

\subsubsection{Interpolation to non-gridded positions}

After building a grid of shears and convergences, users can interpolate them to arbitrary positions within the grid. However, when using this functionality, some care must be employed to prevent the interpolation procedure from spuriously modifying the shear power spectrum or correlation function at scales of interest.

The key effect of interpolation is to multiply the shear power spectrum by a quantity proportional to the square of the Fourier

\footnotetext{
${ }^{21}$ http: / / www . numpy . org/
}

transform of the interpolant. As a result, we found that all interpolants modify the shear 2-point functions in a significant way (>10\% but sometimes much more) for scales below 3 times the original grid spacing. Thus, when interpolating shears to random points, the grid spacing should be chosen with care, keeping in mind the minimum scale above which the shear two-point functions should be preserved. Edge effects can also be important depending on the intended use for the resulting interpolated shears and the choice of interpolant (see Table 1; §3.3.3).

\subsection{Lensing by individual dark matter halos}

The NFWHalo class can produce shears and convergences corresponding to a dark matter halo following a spherical NFW (Navarro et al., 1996) profile, using analytic formulae from Bartelmann (1996) and Wright \& Brainerd (2000). The formulae depend on the cosmology being assumed, which in GALSIM is allowed to be an arbitrary $\Lambda \mathrm{CDM}$ cosmology. It would not be difficult to extend this to more sophisticated cosmological models.

The NFW lensing halo is defined in terms of its mass, concentration, redshift, and position in the image. Then for any given position and redshift of the source galaxy to be lensed, the appropriate lensing quantities can be computed analytically. It is, however, important to note that the implementation in GALSIM adopts the coordinate frame of the observed galaxies, i.e. no deflection angles are calculated or applied to galaxy positions. As a consequence the density of galaxies behind NFW halos is not altered as would happen in nature. If needed, e.g. for studies of magnification from galaxy clusters, this effect should be taken into account.

\section{World coordinate systems}

The galaxy models described above are generally defined in terms of angular units on the sky. For example, a Sérsic profile might be constructed to have a half-light radius of 1.6 arcsec. Before rendering this object onto an image, one must specify how the image pixel coordinates relate to sky coordinates (also known as world coordinates).

The simplest such relationship is to assign a pixel scale to the image, typically in units of arcsec/pixel. However, this is not the only possible such relationship; in general, the "world coordinates" on the sky can be rather complicated functions of the image coordinates. GALSIM allows for a variety of "world coordinate systems" (WCS) ranging from a simple pixel scale to the kinds of WCS functions typically found in the FITS (Flexible Image Transport System, see e.g. Pence et al., 2010) headers of real data images.

\subsection{Types of WCS functions}

The WCS classes used by GALSIM can be broken into two basic types: celestial and euclidean coordinate systems.

Celestial coordinate systems are defined in terms of right ascension (RA) and declination (Dec). In GALSIM this kind of WCS is represented by subclasses of CelestialwCS. This 
includes RaDecFunction, which can represent any arbitrary function the user supplies for $\operatorname{RA}(x, y)$ and $\operatorname{Dec}(x, y)$, and FitsWCS, which reads in the WCS information from a FITS header.

Euclidean coordinate systems are defined relative to a tangent plane projection of the sky. Taking the sky coordinates to be on an actual sphere with a particular radius, the tangent plane would be tangent to that sphere. We use the labels $(u, v)$ for the coordinates in this system, where $+v$ points north and $+u$ points west $^{22}$.

In GALSIM this kind of WCS is represented by subclasses of EuclideanWCS. The most general is UVFunction, which can represent any arbitrary function the user supplies for $u(x, y)$ and $v(x, y)$. AffineTransform is the most general uniform coordinate transformation where all pixels have the same size and shape, but that shape is an arbitrary parallelogram.

Other classes representing restricted specializations of AffineTransform are available. The simplest one, Pixelscale, describes uniform square pixels. To date, this is what has been used in simulations testing shear measurement methods, including the most recent GREAT3 challenge (Mandelbaum et al., 2014), as well as all previous shear testing projects (Heymans et al., 2006; Massey et al., 2007; Bridle et al., 2010; Kitching et al., 2012, 2013). GALSIM therefore makes it easy to ignore all of the WCS options and just use a pixel scale instead. Any function that can take a WCS parameter, can also take a pixel scale parameter, which is interpreted as a Pixelscale WCS.

\subsection{Converting profiles}

It is relatively straightforward to convert a surface brightness profile from the sky coordinates in which it is defined to image coordinates. We only need to assume that the first derivatives of the WCS functions are approximately constant over the size of the object being modelled, which is generally a safe assumption. That is, we use the local linear approximation of the WCS transformation at the location of the object. Currently, GALSIM cannot accurately handle transformations that vary significantly over the size of the profile being rendered; in fact this operation would be very similar to what is required for implementing flexion, and would most simply be incorporated in the context of photon shooting (see $\S 6.3$ ).

The first step if we are dealing with a celestial coordinate system is to do a local tangent projection at the position of the object. Specifically, we use the TAN projection described by Calabretta \& Greisen (2002), but it is likely that any of the tangent plane projections discussed in that work would provide an equivalent conversion to a local Euclidean coordinate system.

The next step is to calculate the Jacobian of the WCS at the location of the object:

$$
\mathbf{J}=\left(\begin{array}{ll}
\mathrm{d} u / \mathrm{d} x & \mathrm{~d} u / \mathrm{d} y \\
\mathrm{~d} v / \mathrm{d} x & \mathrm{~d} v / \mathrm{d} y
\end{array}\right)
$$

\footnotetext{
${ }^{22}$ This can be counterintuitive to some, but it matches the view from Earth. When looking up into the sky, if north is up, then west is to the right.
}

This Jacobian defines the local affine approximation of the WCS at the location of the object, which we assume we can take as uniform over the extent of the profile we need to convert.

Applying a general Jacobian transformation in GALSIM was described above in $\$ 3.4$. Here, we are transforming from $(u, v)$ to $(x, y)$, which means the transformation to be applied is really $\mathbf{J}^{-1}$. To preserve the total flux of the profile, we also need to multiply the resulting profile by $|\operatorname{det} \mathbf{J}|$.

The GALSIM WCS classes have functions toImage and toWorld that effect the conversion in either direction, using whatever subset of the above steps are required for the given WCS. If the WCS transformation is not uniform (so it matters where the object is in the image), then the position of the object (in either coordinate system) must be specified.

\subsection{Integrating over pixels}

While the galaxy profiles are naturally defined in world coordinates, the pixel response is most naturally defined in image coordinates, where it can typically be modelled as a unit square top hat profile. The PSF profile may be more naturally considered in either coordinate system depending on where the model is coming from. Thus, careful attention is required to handle all of these correctly when using a complicated WCS.

We start by ignoring the PSF to show how to properly handle a galaxy drawn onto uniform square pixels of dimension $s$ in an image (corresponding to a Pixelscale WCS). Let the galaxy surface brightness be given in world coordinates as $I^{w}(u, v)$. According to the methods in the previous section, the corresponding profile in image coordinates would be

$$
I^{i}(x, y)=s^{2} I^{w}(x s, y s)
$$

where $s^{2}$ is the $\mid$ det $\mathbf{J} \mid$ factor mentioned above, which ensures that the integral of each version of the profile gives the same total flux.

The pixel response in the two coordinate systems is given by a 2-dimensional top hat function with unit flux:

$$
\begin{aligned}
& P^{w}(u, v)=T_{s}(u, v) \equiv \begin{cases}\frac{1}{s^{2}} & |u|<s / 2,|v|<s / 2 \\
0 & \text { otherwise }\end{cases} \\
& P^{i}(x, y)=T_{1}(x, y)
\end{aligned}
$$

in world and image coordinates respectively, where in the latter the pixel size is, by definition, equal to 1 .

When observed on an image, the galaxy's surface brightness profile is integrated over the area of the pixel. In a particular pixel $(i, j)$, the integrated flux $I_{i j}$ is

$$
\begin{aligned}
I_{i j} & =\int_{i s-s / 2}^{i s+s / 2} \int_{j s-s / 2}^{j s+s / 2} I^{w}(u, v) \mathrm{d} u \mathrm{~d} v \\
& =\int_{-\infty}^{+\infty} \int_{-\infty}^{+\infty} s^{2} I^{w}(u, v) P^{w}(i s-u, j s-v) \mathrm{d} u \mathrm{~d} v \\
& =s^{2}\left(I^{w} \circ P^{w}\right)(i s, j s) \\
& =\int_{-\infty}^{+\infty} \int_{-\infty}^{+\infty} I^{i}(x, y) P^{i}(i-x, j-y) \mathrm{d} x \mathrm{~d} y
\end{aligned}
$$




$$
=\left(I^{i} \circ P^{i}\right)(i, j)
$$

We thus find that $I_{i j}$ is the convolution of the galaxy profile with the pixel response evaluated at the centre of the pixel, and then multiplied by the pixel area. Furthermore, this calculation can be done in either coordinate system. This well-known result (e.g. Lauer, 1999) is the basis of how GALSIM implements the integration of a surface brightness profile over the pixel area.

For more complicated WCS functions, we can still apply the same procedure. We either convert the galaxy profile to image coordinates and convolve by a unit square pixel, or convert the unit square pixel to world coordinates and do the convolution there. In GALSIM the drawImage method that performs the rendering has access to the WCS of the image, and this convolution is handled automatically. The pattern adopted in the code is to transform the galaxy profile into image coordinates and then convolve by a unit square pixel, but the converse would have been equally valid.

For the PSF, when using a non-trivial WCS, some care is required regarding the coordinate system in which the PSF profile is defined. The processes that cause the image coordinates to become distorted from a square pixel are related to the causes of the PSF, namely the atmosphere and the optics of the telescope. Therefore, when choosing to apply a distorted WCS, care is needed about whether the PSF is defined in world or image coordinates. If it is defined in image coordinates, then it should be converted to world coordinates (as described in §5.2) prior to convolution by the galaxy profile.

Finally, if a PSF is measured from an image, such as a real data image of a star, then it already includes a convolution by a pixel response ${ }^{23}$. To apply this consistently to simulated data using the same WCS and pixel scale, an additional convolution by a pixel should not be applied, since that would effectively convolve by the pixel response twice. This kind of rendering without the extra pixel convolution is possible in GALSIM using the no_pixel option of drawImage.

\section{Image rendering}

A given object can be rendered onto an image ("drawn") through three methods in GALSIM: direct drawing in real space; drawing via discrete Fourier transform (DFT); or drawing via "photon shooting," whereby the surface brightness profile is treated as a probability distribution, and a finite number of "photons" sampled from this distribution are "shot" onto the image.

In principle all three rendering methods should be equivalent, apart from the shot noise that is inherent to the photon-shooting method. However, there is one additional difference that is worth noting. The photon-shooting method bins the photons according to the pixels they fall into and hence automatically

\footnotetext{
${ }^{23}$ It is possible to remove the pixel response from the PSF image with the Deconvolve operator in GALSIM which deconvolves by the original pixel. This will only be numerically viable if the resulting object is drawn onto an image with pixels that are either the same size or (preferably) larger.
}

integrates the profile over the pixels. Thus, the no_pixel option of drawImage mentioned above in $\$ 5.3$ will always use either direct rendering or DFT as appropriate.

There are in practice also further minor differences between the rendered output of the different methods that are due to approximations inherent to each technique. These we highlight below.

Not all objects can be rendered with all three methods. In particular, convolution in real space is only implemented for convolution of two profiles, one of which is typically the pixel response (cf. §6.1.5). Deconvolution (see $§ 3.5$; §6.5) is only possible with the DFT method. Non-affine transformations such as flexion (Goldberg \& Bacon, 2005; Irwin \& Shmakova, 2005; Bacon et al., 2006) cannot be done in Fourier space; flexion is not currently implemented in GALSIM for this reason. Table 2 summarizes the advantages and disadvantages of each rendering method.

\subsection{Direct rendering}

The default way to draw an object in GALSIM is to integrate its surface brightness over the pixel response by convolving the object's profile with a square pixel (cf. §5.3). This means the object that is actually being drawn will really be a convolution, which is normally rendered using the DFT method (\$6.2).

However, it is possible to tell GALSIM not to convolve by the pixel and instead draw the profile directly by sampling the surface brightness at the centre of each pixel. This is the easiest rendering method to understand, so we describe it first. But of course, it does not directly correspond to a real image, so the sum of the pixel values may not match the input flux, for instance.

\subsubsection{Analytic objects}

If an analytic surface brightness profile is drawn without convolving by the pixel response, GALSIM will use direct rendering, which just involves calculating $I(x, y)$ at the location of each output pixel. The formulae for $I(x, y)$ for our various analytic objects were given in $\S 3$, and they are summarized in Table 3.

Many objects have an analytic formula in real space, so the direct rendering is straightforward. However, the Kolmogorov profile is only analytic in Fourier space, so the implementation of $I(r)$ in real space uses a cubic spline lookup table for the Hankel transform of $\widetilde{I}(k)$. This calculation is done the first time a Kolmogorov object is instantiated and saved for any further instantiations, so the setup cost is only required once.

For the radially symmetric profiles, we can take advantage of the known symmetry to speed up the calculation. There are also usually vectorization techniques that lead to further improvements in efficiency.

\subsubsection{Shapelet profiles}

The shapelet functions are not radially symmetric, but the code to directly render Shapelet objects uses fast recurrence relations for the shapelet functions given in Bernstein \& Jarvis 
Table 2: Characteristics of different rendering methods

\begin{tabular}{|c|c|c|c|}
\hline Characteristic & Direct real-space & Fourier & Photon shooting \\
\hline Operations for setup $^{a}$ & 0 & $O\left(N^{2} \log N\right)$ & $O\left(N^{2} \log N\right)$ \\
\hline Operations for rendering ${ }^{a}$ & $O\left(N^{2}\right)$ & $O\left(N^{2} \log N\right)$ & $O\left(N_{\gamma} \log N\right)$ \\
\hline Operations for addition $^{\mathrm{a}}$ & $O\left(N^{2}\right)$ & $O\left(N^{2}\right)$ & $O\left(N_{\gamma}\right)$ \\
\hline Operations for convolution ${ }^{\mathrm{a}}$ & $O\left(N^{4}\right)^{\mathrm{b}}$ & $O\left(N^{2}\right)$ & $O\left(N_{\gamma}\right)$ \\
\hline Operations for deconvolution ${ }^{\mathrm{a}}$ & impossible & $O\left(N^{2}\right)$ & impossible \\
\hline Easy to apply affine transformations? & yes & yes & yes \\
\hline Easy to apply non-affine transformations? & yes & no & yes \\
\hline Inaccuracies & - & band-limiting, folding & shot noise, truncation \\
\hline Fastest for & analytic objects & $\operatorname{high} S / N$ & low $S / N$ \\
\hline
\end{tabular}

Table 3: Analytic GALSIM objects

\begin{tabular}{|c|c|c|c|}
\hline Class name & Real-space formula ${ }^{a}$ & Fourier-space formula ${ }^{a}$ & Photon-shooting method $^{\mathrm{b}}$ \\
\hline Exponential & $e^{-r / r_{\mathrm{s}}}$ & $\left(1+k^{2} r_{\mathrm{s}}^{2}\right)^{-3 / 2}$ & Interval \\
\hline DeVaucouleurs & $e^{-7.669\left(r / r_{\mathrm{e}}\right)^{1 / 4}}$ & LUT & Interval \\
\hline Sersic & $e^{-b(n)\left(r / r_{\mathrm{e}}\right)^{1 / n}}$ & LUT & Interval \\
\hline Airy & {$\left[\frac{J_{1}(v)-\epsilon J_{1}(\epsilon v)}{v}\right]^{2}, \quad v=\pi r D / \lambda$} & Analytic $^{c}$ & Interval \\
\hline Moffat & $\left(1+\frac{r^{2}}{r_{0}^{2}}\right)^{-\beta}$ & $\mathrm{LUT}^{\mathrm{d}}$ & Analytic \\
\hline Kolmogorov & LUT & $e^{-\left(k / k_{0}\right)^{5 / 3}}$ & Interval \\
\hline Gaussian & $e^{-r^{2} / 2 \sigma^{2}}$ & $e^{-k^{2} \sigma^{2} / 2}$ & Analytic \\
\hline Shapelet & See $\S 3.3 .2$ & See $\S 3.3 .2$ & Not implemented \\
\hline Pixel & $1,|x|<s / 2,|y|<s / 2$ & $\operatorname{sinc}\left(s k_{x}\right) \operatorname{sinc}\left(s k_{y}\right)$ & Analytic \\
\hline
\end{tabular}

${ }^{a}$ Normalization factors have been omitted from the given formulae for brevity; the integral of the real-space profile and the $k=0$ value in Fourier space should both equal the flux $F$ of the object. "LUT" means that the function requires integrations that are precomputed and stored in a cubic-spline look-up table.

${ }^{b}$ The photon-shooting methods are the following: "Analytic" means that photon locations are derived from a remapping of one or more uniform deviates into $\mathbf{x}$;

"Interval" involves a combination of weighting and rejection sampling, as described in Section 6.3; "Not implemented" means that GALSIM cannot currently render this with photon shooting.

${ }^{c}$ Fourier representation of the Airy function with obscuration is analytic but too complex for the table.

${ }^{\mathrm{d}}$ For generic $\beta$ values, a lookup table is used. However, some particular values of $\beta$ have analytic Fourier-space formulae, which GALSIM uses in these cases. 
(2002), which vectorize conveniently when applied to the pixels in the image. They are thus also relatively efficient to draw.

\subsubsection{Interpolated images}

InterpolatedImage objects are usually the slowest to directly render, since equation (25) involves a sum over a number of the original pixels for each output pixel value, the exact number depending on the interpolant being used (see Table 1).

\subsubsection{Transformations}

All of the transformations described in $\$ 3.4$ are very simple to apply when directly rendering. The value of the transformed profile, including all three kinds of effects, is simply

$$
I^{\prime}(\mathbf{x})=c I\left[\mathbf{A}^{-1}(\mathbf{x}-\mathbf{x} \mathbf{0})\right] .
$$

where $I(\mathbf{x})$ is the original profile.

\subsubsection{Compositions}

Directly rendering a sum of profiles is trivial, since each profile can be rendered individually, adding the flux to whatever has already been rendered.

The rendering of a convolution is almost always done via DFT or photon-shooting methods. However, when convolving two objects that are both known to have high-frequency content, such that an unaliased DFT is impractical, GALSIM may elect to render it directly through a Gauss-Kronrod-Patterson evaluation of the convolution integral, equation (29).

Generally such circumstances do not represent any physically realizable image, since real telescopes and detectors lead to band-limited images. However, in simulated images, it can happen if two objects being convolved both have hard edges. Here, a "hard edge" means a place where the surface brightness abruptly drops from some finite value to zero. For example a $\mathrm{Pixel}$ convolved with a truncated Moffat profile would by default be convolved using direct integration. In this and similar cases, the direct integration is generally both faster and more accurate.

\subsection{Discrete Fourier transform rendering}

When rendering images that include convolution by a pixel response (and often other profiles such as a PSF), GALSIM uses DFTs by default.

\subsubsection{Band limiting and folding}

To sample a surface brightness profile $I(\mathbf{x})$ onto an $M \times M$ grid with sample pitch $\Delta x$ via DFT, we will need to know its Fourier transform $\widetilde{I}(\mathbf{k})$ at all points with $k_{x}, k_{y}$ being multiples of $\Delta k=2 \pi /(M \Delta x)$. If any frequency modes are present in $\widetilde{I}(\mathbf{k})$ beyond the Nyquist frequency $\pi / \Delta x$ of the output grid these will be aliased in the output image, as required for the correct rendering of undersampled data.

The input to the DFT will be the $M \times M$ Hermitian array of Fourier coefficients summed over all aliases:

$$
\left.\widetilde{a}_{i j}=\sum_{p, q} \widetilde{I}[(i+p M) \Delta k,(j+q M) \Delta k)\right],
$$

$$
-M / 2 \leq i, j<M / 2
$$

These sums must be truncated at some finite $(p, q)$ range. We must select a spatial frequency $k_{\max }$ such that we approximate $\widetilde{I}\left(k_{x}, k_{y}\right)=0$ for $\left|k_{x}\right|>k_{\max }$ or $\left|k_{y}\right|>k_{\max }$. Any real telescope produces bandlimited images, transmitting zero power beyond a wave vector of $k_{\max }=2 \pi D / \lambda$, where $D$ is the maximum entrance aperture dimension and $\lambda$ is the wavelength of observation. For space-based observations we are typically creating images sampled near the Nyquist scale of $\lambda / 2 D$ for this frequency, and we can run the sum (46) over all physically realizable frequencies without approximation.

For ground-based observations, the PSF is typically much larger than the diffraction limit, and the pixel scale has Nyquist frequency well below the physical $k_{\max }$. To render images with acceptable speed it is often necessary to approximate the Fourier transform $\widetilde{I}(\mathbf{k})$ as being identically zero beyond a chosen $k_{\max }$. This is called band-limiting the image. Common idealizations of PSFs, such as the Gaussian, Moffat, and Kolmogorov functions, are unbounded in $\mathbf{k}$, and therefore we must select a $k_{\max }$ which yields an acceptable approximation.

Every kind of surface brightness profile in GALSIM is able to calculate and return its $k_{\max }$, which we take to be the value of $k$ where the Fourier-domain profile drops below a threshold of $10^{-3}$ of the peak. See $\$ 6.4$ for more detail about the parameter (maxk_threshold) that controls this value. For some profiles $k_{\max }$ can be calculated analytically; where this is not possible $k_{\max }$ is determined numerically using an appropriate method for each profile.

Another characteristic of the DFT is that each output value $I_{i j}=I(i \Delta x, j \Delta x)$ is actually the true function folded at the pe$\operatorname{riod} P=M \Delta x=2 \pi / \Delta k$ :

$$
I_{i j}=\sum_{p, q} I(i \Delta x+p P, j \Delta x+q P)
$$

Since it is impossible for $I(\mathbf{x})$ to be compact after being bandlimited, this leads to some degree of inaccuracy due to folding of the image. We can limit the aliasing errors by ensuring that every DFT is done with a sufficiently large period. Every surface brightness profile in GALSIM also knows what $k_{\text {step }}$ value should be used for the DFT (cf. folding_threshold in $\S 6.4)$. The DFT is executed with period $P \geq 2 \pi / k_{\text {step }}$, implying $\Delta k \leq k_{\text {step }}$ and $M \geq 1 / k_{\text {step }} \Delta x$.

If the user has requested rendering of an image that does not meet this criterion, GALSIM will execute the DFT on a grid of larger $M$ that does, and then extract the output image from the result of this larger DFT. If the user does not specify an image size, GALSIM will select a value that satisfies this bound.

The DFT image must have dimension $M \geq 2 k_{\max } / k_{\text {step. }}$. Some of the GALSIM profiles contain sharp features that cause this minimum $M$ to exceed the maximum allowed DFT size, in particular the Pixel class and the Sersic class with higher indices, including the DeVaucouleurs class. GALSIM will raise an error if one attempts to render such an object via DFT without first convolving by another object that attenuates the high-frequency information. This should not be an issue for rendering of realistic images, in which convolution with the 
PSF and pixel response will typically limit the bandwidth of the image to a manageable value.

\subsubsection{Analytic objects}

Table 3 lists the profiles for which GALSIM calculates Fourier-domain values analytically.

For those radial profiles without fast analytic formulae in Fourier domain, we tabulate numerical transforms into a lookup table and use cubic spline interpolation to return $\widetilde{I}(k)$. The initial setup of these tables can take some time, but they are cached so that later Fourier-domain evaluations of the same type of profile are accomplished in constant time.

At small values of $k=|\mathbf{k}|$ we generally replace the spline interpolation with an analytic quadratic (or higher) order Taylor expansion of $\widetilde{I}(k)$, because the behaviour of $\widetilde{I}(\mathbf{k})$ near the origin has a strong effect on the appearance of poorly-resolved objects. In particular the second derivative of $\widetilde{I}(\mathbf{k})$ determines the second central moments that are critical for measurement of weak gravitational lensing, and the spline interpolation can produce incorrect derivatives at the origin.

\subsubsection{Shapelet profiles}

Since shapelets are their own Fourier transforms (cf. equation (24)), the code to draw a Shapelet object in Fourier space is essentially identical to the code to directly render it. It uses fast recurrence relations, which are efficient when applied to the pixels in the Fourier image.

\subsubsection{Interpolated images}

Given an $N \times N$ array of samples at pitch $\Delta x$, we can perform a DFT to yield samples of the Fourier transform at a grid of values $\mathbf{k}_{i j}=(i / N \Delta x, j / N \Delta x)$. To render this function back onto a different $x$-domain grid, or to execute transformations, we need to evaluate $\widetilde{I}(\mathbf{k})$ at arbitrary $\mathbf{k}$ between the grid of DFT values. This requires some $k$-space interpolation scheme.

Furthermore we need to account for the facts that (a) the interpolated image is finite in extent, while the DFT will yield the transform of a periodic function, and (b) it represents a continuous, interpolated version of the input sample grid. Thus two distinct interpolants are required: the $x$-domain interpolant $K_{x}$ is chosen by the user and is part of the definition of $I(\mathbf{x})$; the $k$ domain interpolant $K_{k}$ is used to generate $\widetilde{I}(\mathbf{k})$ by interpolating over the DFT of the input image and the Fourier transform of $K_{x}$.

Correct evaluation of the interpolated function in $k$-space is not trivial. Bernstein \& Gruen (2014) present a detailed description of the steps required, and we summarize their results here. First, they find that the rigorously correct form of $K_{k}$ is a sinc function wrapped at the extent of the $x \rightarrow k$ DFT. Since this interpolant spans the entire plane, it means that the interpolation of $\widetilde{I}(\mathbf{k})$ to all the $M \times M$ points needed for the DFT requires $N^{2} M^{2}$ operations, which can be prohibitively slow. As a consequence we normally elect to approximate this using a compact interpolant for $K_{k}$.

Bernstein \& Gruen (2014) demonstrate that the consequences of this interpolation are a slight multiplicative scaling of the image, plus the generation of 4 "ghost images" displaced by $N \Delta x$ along each axis. They find that these artifacts can be reduced below 0.001 of the input flux by a combination of zeropadding the input image by $4 \times$ in each direction before conducting the $x \rightarrow k$ DFT, producing a denser set of samples in $k$ space, and then using a custom-designed, 6-point piecewise quintic polynomial interpolant for $K_{k}$. This recipe is the default for Fourier-domain rendering of the InterpolatedImage in GALSIM. Users can override the default 4-fold zero-padding factor and/or the choice of the Quintic interpolant when intializing an InterpolatedImage object.

The value of $k_{\text {step }}$ for an interpolated image of input size $N$ and pitch $\Delta x$ would naively be set at $2 \pi /(N \Delta x)$ to reflect the bounded size of the input image. We must recall, however, that the interpolation of the input image by the kernel $K_{x}$ will extend the support of the object and slightly lower the desired $k_{\text {step }}$ (with the sinc interpolant extending the footprint of the image enormously).

The value of $k_{\max }$ for a raw sampled image is infinite because it is composed of $\delta$ functions. However the application of the interpolant $K_{x}$ to the samples will cause $\widetilde{I}(\mathbf{k})$ to roll off yielding $k_{\max }=c \pi / \Delta x$, where $c$ is a constant characteristic to the chosen $K_{x}$. In this respect the band-limited sinc interpolant, with $c=1$, is ideal, but the Lanczos interpolants with $n=3-$ 5 offer much more compact support with $2<c<3$ sufficing to contain all aliases with amplitude $>0.001$. See Table 1 in Bernstein \& Gruen (2014) for the relevant characteristics of the interpolants.

\subsubsection{Transformations}

Transformations of functions have well-known correspondents in Fourier domain, which we quickly review here.

A shift $I^{\prime}(\mathbf{x})=I\left(\mathbf{x}-\mathbf{x}_{0}\right)$ has Fourier-domain equivalent $\widetilde{I^{\prime}}(\mathbf{k})=e^{\mathrm{i} \mathbf{k} \cdot \mathbf{x}_{0}} \widetilde{I}(\mathbf{k})$. A shift leaves $k_{\max }$ and $k_{\text {step }}$ unchanged.

Linear transformations of the plane, such that $I^{\prime}(\mathbf{x})=$ $I\left(\mathbf{A}^{-\mathbf{1}} \mathbf{x}\right)$, are equivalent to $\widetilde{I^{\prime}}(\mathbf{k})=\widetilde{I}\left(\mathbf{A}^{\mathbf{T}} \mathbf{k}\right)$. We identify the maximum and minimum eigenvalues $\lambda_{+}$and $\lambda_{-}$of $\mathbf{A}$, and set

$$
\begin{aligned}
k_{\max } & \rightarrow \lambda_{-}^{-1} k_{\max } \\
k_{\text {step }} & \rightarrow \lambda_{+}^{-1} k_{\text {step }}
\end{aligned}
$$

\subsubsection{Compositions}

The Fourier transform is linear, so that if $I(\mathbf{x})=a I_{A}(\mathbf{x})+$ $b I_{B}(\mathbf{x})$, we also have $\widetilde{I}(\mathbf{k})=a \widetilde{I}_{A}(\mathbf{k})+b \widetilde{I}_{B}(\mathbf{k})$. When we are dealing with a sum of multiple components $I_{i}$, we set

$$
\begin{aligned}
k_{\text {max }} & =\max _{i}\left(k_{\text {max }, i}\right) \\
k_{\text {step }} & =\min _{i}\left(k_{\text {step }, i}\right)
\end{aligned}
$$

The convolution $I(\mathbf{x})=\left(I_{A} \circ I_{B}\right)(\mathbf{x})$ is equivalent to $\widetilde{I}(\mathbf{k})=$ $\widetilde{I}_{A}(\mathbf{k}) \widetilde{I}_{B}(\mathbf{k})$. Under a convolution of multiple elements, we set

$$
\begin{aligned}
& k_{\max }=\min _{i}\left(k_{\max , i}\right) \\
& k_{\text {step }}=\left(\sum_{i} k_{\text {step }, i}^{-2}\right)^{-1 / 2}
\end{aligned}
$$


While the propagation of the band limit $k_{\max }$ is rigorously correct for convolution, the propagation of $k_{\text {step }}$ in equation (53) is merely heuristic. It is based on the exact statement that the central second moments of objects sum in quadrature under convolution. But there is no general rule for the propagation of the radius enclosing some chosen fraction of the total object flux, so our heuristic is known to be correct only for Gaussian objects. For strictly compact functions such as a $\mathrm{P}$ ixel, the maximum nonzero element $x_{\max }$ actually adds linearly, not in quadrature, under convolution. GALSIM provides the option to manually increase the size of DFTs if one is worried that the $k_{\text {step }}$ heuristic will lead to excessive aliasing.

Finally, a Deconvolve operation applied to some image $I_{A}(\mathbf{x})$ yields $1 / \widetilde{I}_{A}(\mathbf{k})$ in the Fourier domain. We leave $k_{\max }$ and $k_{\text {step }}$ unaltered, because the deconvolved object is usually illdefined unless it is later re-convolved with an object that has a smaller $k_{\max }$ value.

\subsection{Photon shooting}

"Photon shooting" was used successfully by Bridle et al. (2009, 2010) and Kitching et al. (2012, 2013) to generate the simulated images for the GREAT08 and GREAT10 weak lensing challenges. The objects were convolutions of elliptical Sérsic-profile galaxies with Moffat-profile PSFs. GALSIM extends this technique to enable photon shooting for nearly all of its possible objects, except for deconvolutions.

When we "shoot" a GALSIM object, $N_{\gamma}$ photons are created with weights $w_{i}$ and positions $\mathbf{x}_{i}$. The total weight within any region has an expectation value of the flux in that region, and the total weights in any two regions are uncorrelated ${ }^{24}$.

We allow for non-uniform weights primarily so that we can represent negative values of surface brightness. This is necessary to realize interpolation with kernels that have negative regions (as will any interpolant that approximates band-limited behaviour), and to correctly render interpolated images that have negative pixel values, such as might arise from using empirical, noisy galaxy images.

For photon shooting to be possible on a GALSIM object, it must be able to report $f_{\text {pos }}$ and $f_{\text {neg }}$, the absolute values of the flux in regions with $I(\mathbf{x})>0$ and $I(\mathbf{x})<0$, respectively. When $N_{\gamma}$ photons are requested, we draw their positions from the distribution defined by $|I(\mathbf{x})|$ and then assign weights

$$
w_{i}=\frac{f_{\mathrm{abs}}}{N_{\gamma}} \operatorname{sign}\left[I\left(\mathbf{x}_{i}\right)\right]=\frac{f_{\mathrm{pos}}+f_{\text {neg }}}{N_{\gamma}} \operatorname{sign}\left[I\left(\mathbf{x}_{i}\right)\right] .
$$

Shot noise in the fraction of photons that end up in the negative-brightness regions will lead to variations in the total flux of the photons. GALSIM mostly accounts for this effect automatically by selecting an appropriate number of photons to get the noise correct (cf. §6.3.6). However, the partial cancellations between positive- and negative-flux photons means that

24 This will not be true if you turn off the Poisson variation in the total flux, which is optional but turned on by default, since then the fixed total flux will lead to some correlation in the pixel values. the resulting noise will not actually be distributed precisely according to a Poisson distribution for the given flux; only the variance of the noise will be approximately accurate. For objects constructed purely from positive-flux profiles, this effect is absent, and the noise is indeed Poisson.

We will see below that in some cases, $\left|w_{i}\right|$ is allowed to depart slightly from the constant $f_{\text {abs }} / N_{\gamma}$ value, which also alters the noise properties slightly.

\subsubsection{Analytic objects}

Photon shooting a surface-brightness function $I(\mathbf{x})$ is simplest when there is a known transformation from the unit square or circle to the plane whose Jacobian determinant is $\propto I$. $\mathrm{Pixel}$ is the simplest such case, since it is just a scaling of the unit square.

Many other profiles are circularly symmetric functions with a cumulative radial distribution function

$$
C(r)=\frac{\int_{0}^{r} I\left(r^{\prime}\right) r^{\prime} \mathrm{d} r^{\prime}}{\int_{0}^{\infty} I\left(r^{\prime}\right) r^{\prime} \mathrm{d} r^{\prime}}
$$

If $u$ is a uniform deviate between 0 and $1, C^{-1}(u)$ will be distributed as $r I(r)$, the distribution of flux with radius. A second uniform deviate can determine the azimuthal angle of each photon.

GALSIM uses fast analytic $C^{-1}(u)$ functions for the Gaussian (e.g. Press et al., 1992) and Moffat classes.

For the other circularly symmetric profiles we use the following algorithm to convert a uniform deviate into the radial flux distribution of $|I|$. The algorithm is provided with the function $I(r)$ and with a finite set of points $\left\{R_{0}, R_{1}, R_{2}, \ldots, R_{M}\right\}$ with the guarantee that each interval $\left(R_{i}, R_{i+1}\right)$ contains no sign changes and at most one extremum of $I(r)$. The absolute flux $\left|f_{i}\right|$ in each annulus is obtained with standard numerical integration techniques.

Note that this algorithm requires a maximum radius, so the photon shooting rendering requires truncation of unbounded profiles. The fraction of excluded flux due to this truncation is given by the shoot_accuracy parameter described in $\$ 6.4$.

The algorithm proceeds as follows:

1. The code first inserts additional nodes into the $R_{i}$ series at the locations of any extrema.

2. The radial intervals are recursively bisected until either the absolute flux in the interval is small, or the ratio $\max |I| / \min |I|$ over the interval is below a chosen threshold.

3. The integral $f_{i}$ of the absolute flux is calculated for each annular interval along with the probability $p_{i}=f_{i} / \sum_{i} f_{i}$ of a photon being shot into the interval. We can also calculate the cumulative probability $P_{i}=\sum_{j<i} p_{j}$ of the photon being interior to the annulus.

4. For each photon we draw a uniform deviate $u$. It is mapped to a radius $r$ by finding the interval $i$ such that $P_{i} \leq u<$ $P_{i+1}$ and then assigning

$$
r^{2}=R_{i}^{2}+\frac{u-P_{i}}{p_{i}}\left(R_{i+1}^{2}-R_{i}^{2}\right) .
$$


In other words we draw $r$ from a flux distribution that approximates $|I(r)|$ as a "wedding cake" of constant-valued annuli.

5. (a) If $r$ is in an interval that has a narrow range of brightness variation, we re-weight the photon by a factor equal to the brightness at $r$ relative to the mean brightness in the annulus:

$$
w=\frac{f_{\mathrm{abs}}}{N_{\gamma}} \frac{I(r) \pi\left(R_{i+1}^{2}-R_{i}^{2}\right)}{f_{i}} .
$$

(b) If the range of variation of $I$ in the annulus is large, which can happen when the annulus is near a zero crossing, we implement rejection sampling by drawing another uniform deviate $u^{\prime}$, and keeping the photon $r$ if $|I(r)| / \max |I|>u^{\prime}$. If the photon is rejected, we use another uniform deviate $u$ to select a new trial radius within the interval:

$$
r^{2}=R_{i}^{2}+u\left(R_{i+1}^{2}-R_{i}^{2}\right)
$$

The rejection test is repeated, and the process is iterated until a photon radius is accepted. This photon is given the nominal weight (54).

6. The azimuthal angle of the photon is selected with a uniform deviate.

This procedure yields $N_{\gamma}$ photon locations and weights, employing $2 N_{\gamma}(1+\epsilon)$ uniform deviates and $N_{\gamma}(1+\epsilon)$ evaluations of $I(r)$, where $\epsilon$ is the fraction of photons rejected in step 5b. We note that the finite widths of the annuli must be small for the approximation to be good: the close correspondence of photon shooting and DFT rendering results in $\$ 9.1$ demonstrates that this is achieved in GALSIM.

In practice we re-order the intervals into a binary tree (see, e.g., Makinson, 2012) to optimize the selection of an interval with the initial uniform deviate. With this tree structure the mean number of operations to select an interval approaches the optimal $-\sum p_{i} \log p_{i}<\log M$, where $M$ is the final number of radius intervals.

The Sersic, Exponential, DeVaucouleurs, Airy, and Kolmogorov classes use this interval-based photon shooting algorithm. The construction of the interval structure and flux integrals are done only once for the Exponential and DeVaucouleurs profiles and once for each distinct Sérsic index $n$ for Sersic profiles or distinct central obscuration for Airy. These results are cached for use by future instances of the classes in each case.

\subsubsection{Shapelets}

Because shapelet profiles are not radially symmetric, implementing photon shooting for such objects represents a significant challenge (although it is far from impossible). As of GALSIM version 1.1, we have not attempted to support photon shooting for Shapelet objects.

\subsubsection{Interpolated images}

Photon shooting an Interpolated Image object requires two steps: first we distribute the photons among the grid points $(i \Delta x, j \Delta x)$ with probabilities $p_{i j}=\left|a_{i j}\right| / \sum\left|a_{i j}\right|$. We select an ordering for these probabilities and create the cumulative probabilities $P_{i j}$. For each photon, we draw a uniform deviate $u$, and assign an $(i, j)$ value as the last one in the ordering with $P_{i j}<u$. The weight is given the sign of $a_{i j}$. As we did with the radial function intervals above, we create a tree structure which reduces the selection of each $(i, j)$ to $O(\log N)$ for an $N \times N$ input image.

The second step in photon shooting the InterpolatedImage is to convolve the gridded samples with the interpolation kernel $K_{x}$ by adding to each photon's location a displacement drawn from the kernel. Since our 2D interpolants are all separable into 1D functions, we can draw an $x$ and $y$ displacement from the 1D functions. The $\delta$-function interpolant can of course skip this step, and the nearest-neighbour and linear interpolants are trivially generated from uniform deviates. Photon shooting is implemented for the other interpolants using the same algorithm described for the radial analytic functions, with the replacement of $r^{2} \rightarrow x$ in equations (56) \& (57) because of the linear instead of circular geometry.

Attempts to shoot photons through an image with a sinc interpolant will throw an exception. The long oscillating tail of the sinc function means that $f_{\text {abs }}$ is divergent.

Photon shooting an InterpolatedImage thus requires $3 N_{\gamma}(1+\epsilon)$ random deviates plus, for most interpolants, $2 N_{\gamma}(1+$ $\epsilon)$ evaluations of the 1D kernel function.

\subsubsection{Transformations}

All the transformations described in $\$ 3.4$ are very simply implemented in photon shooting. Flux rescaling is simply a rescaling of the $w_{i}$. Any transformation of the plane can be executed by $\mathbf{x}_{i} \rightarrow T\left(\mathbf{x}_{i}\right)$.

\subsubsection{Compositions}

To shoot $N_{\gamma}$ photons from a sum of $M$ profiles, we draw the number of photons per summand $\left\{n_{1}, n_{2}, \ldots, n_{M}\right\}$ from a multinomial distribution with expectation value $\left\langle n_{j}\right\rangle=$ $N_{\gamma} f_{\mathrm{abs}, j} / \sum_{j} f_{\mathrm{abs}, j}$. Then we concatenate the lists of $n_{i}$ photons generated by shooting the individual summands.

Convolution is similarly straightforward: to shoot $N_{\gamma}$ photons through $I_{A} \circ I_{B}$, we draw $N_{\gamma}$ photons each from $I_{A}$ and $I_{B}$, set the output weights to $w_{i}=w_{i, A} w_{i, B}$, and sum the positions to get $\mathbf{x}_{i}=\mathbf{x}_{i, A}+\mathbf{x}_{i, B}$. To ensure that there is no correlation between the photon positions of $A$ and $B$ in the sequence, GALSIM randomizes the order of the $B$ photons. This procedure generalizes straightforwardly to the case of convolving more than two profiles together.

We are not aware of a practical means of implementing deconvolution via photon shooting, so GALSIM cannot use photon shooting for any profile that involves a deconvolution.

\subsubsection{Selecting an appropriate number of photons}

One of the main advantages of using the photon-shooting method for rendering images is that for objects with low signalto-noise $(S / N)$, it can be much faster to shoot a relatively small number of photons compared to performing one or more Fourier transforms. However, for this to be effective, one must 
know how many photons to shoot so as to achieve the desired final $S / N$ value.

The simplest case is when there are no components that require negative-flux photons. Then the flux $f$ of the object given in photons ${ }^{25}$ is exactly equal to the number of photons that would be detected by the CCD. In such a case, the flux itself provides the number of photons to shoot.

This simple procedure is complicated by a number of considerations. The first is that the flux itself is a random variable. So by default, GALSIM will vary the total number of photons according to Poisson statistics, which is the natural behaviour for real photons. This means that the actual realized flux of the object varies according to a Poisson distribution with the given mean value. This feature can also be turned off if desired, since for simulations, users may want a specific flux value, rather than just something with the right expectation value.

A more serious complication happens when there are components that require negative-flux photons to be shot, such as those involving interpolants (cf. §6.3.3). We need more photons to get the right total flux, since some of them have negative flux, but then the increased count leads to more noise than we want. The solution we adopt to address this is to have each photon carry somewhat less flux and use even more photons.

Let us define $\eta$ to be the fraction of photons being shot that have negative flux, and let each photon carry a flux of $\pm g$. We want both the total flux shot and its variance to equal the desired flux, $f$ :

$$
\begin{aligned}
f & =N_{\text {photons }}(1-2 \eta) g \\
\operatorname{Var}(f) & =N_{\text {photons }} g^{2}
\end{aligned}
$$

Setting these equal, we obtain

$$
\begin{aligned}
g & =1-2 \eta \\
N_{\text {photons }} & =f /(1-2 \eta)^{2}
\end{aligned}
$$

GALSIM's function drawImage automatically does the above calculation by default when photon shooting, although users can override it and set a different number of photons to be shot if they prefer.

Note that the above calculation assumes that $\eta$ is a constant over the extent of the profile being shot. In reality, it is not constant, which leads to correlations between different regions of a photon-shot image using photons of mixed sign. The resulting noise pattern will roughly resemble the noise of a real photon image, but one should not rely on the noise being either stationary or uncorrelated in this case. Nor will the noise in any pixel follow Poisson statistics. These inaccuracies gets more pronounced as $\eta$ nears 0.5 .

There is one additional feature, which can be useful for objects with moderately high flux, but which are still dominated

25 Technically, in GALSIM the flux is defined in so-called analog-to-digital units, ADU. These are the units of the numbers on astronomical images. We allow a ga in parameter, given in photons/ADU, to convert between these units and the actual number of photons, combining both the normal gain in e-/ADU and the quantum efficiency in e-/photon. The default behaviour is to ignore all these issues and use a gain of 1 . by sky background light (a relatively common use case). It may take many photons to get the noise level correct, a computational cost which is wasted if a larger amount of noise is then added from the sky background. The same final $S / N$ can be obtained (to a very good approximation) by shooting fewer photons, each with flux larger than the normal choice of $g=1-2 \eta$.

To address this, GALSIM has an option to allow the photon shooting process to add an additional noise per pixel over the Poisson noise expected for each pixel. Each pixel may be allowed to have $v$ excess variance. The brightest pixel in the image, with flux $I_{\max }$, can then have a variance of up to $I_{\max }+v$. Thus the number of photons can be reduced by as much as $I_{\max } /\left(I_{\max }+v\right)$. The flux carried by each photon is increased to keep the total flux equal to the target value, $f$ :

$$
\begin{aligned}
N_{\text {photons }} & \geq \frac{I_{\max } f}{\left(I_{\max }+v\right)(1-2 \eta)^{2}} \\
g & =\frac{f}{N_{\text {photons }}(1-2 \eta)}
\end{aligned}
$$

Clearly, this is only helpful if $v \gg I_{\max }$, which is indeed the case when the image is sky noise dominated. This is a common use case in realistic simulations, particularly ones that are meant to simulate ground-based observations.

Users of this behaviour in GALSIM must explicitly indicate how much extra noise per pixel, $v$, is tolerable. Typically this will be something like $1 \%$ of the sky noise per pixel. This is not the default behaviour, because at the time of rendering the image, the code does not know how much (or whether) sky noise will be added.

An additional complication with the above prescription is that the code does not know the value of $I_{\max }$ a priori. So the actual algorithm starts by shooting enough photons to get a decent estimate for $I_{\max }$. Then it continues shooting until it reaches the value given by equation (63), while periodically updating the estimated $I_{\max }$. At the end of this process, it rescales the flux of all the photons according to equation (64) using the final value of $N_{\text {photons }}$.

\subsection{Setting tolerances on rendering accuracy}

Some of the algorithms involved in rendering images involve tradeoffs between speed and accuracy. In general, we have set accuracy targets appropriate for the weak lensing requirements of Stage IV surveys such as LSST, Euclid (Laureijs et al., 2011), and WFIRST (cf. §9). However, users may prefer faster code that is slightly less accurate for some purposes, or more accurate but slower for others. This is possible in GALSIM using a structure called GSP arams, which includes many parameters pertaining to the speed versus accuracy tradeoff, some of which are described below:

- folding_threshold ${ }^{26}$ sets a maximum permitted amount of real space image folding due to the periodic

\footnotetext{
${ }^{26}$ In earlier versions of GALSIM this parameter was named alias_threshold. It was renamed for clarity and to avoid confusion with the phenomenon of aliasing in undersampled data.
} 
nature of DFTs, as described in §6.2.1. It is the critical parameter for defining the appropriate step size $k_{\text {step. }}$. Additionally, it is relevant when letting GALSIM choose output image sizes: the image will be large enough that at most a fraction folding_threshold of the total flux falls off the edge of the image. The default is $5 . e-3$.

- maxk_threshold sets the maximum amplitude of the high frequency modes in Fourier space that are excluded by truncating the DFT at some maximum value $k_{\max }$. As described in $\$ 6.2 .1$, this truncation is required for profiles that are not formally band-limited. Reducing maxk_threshold can help minimize the effect of "ringing" in images for which this consideration applies. The default is $1 . e-3$.

- xvalue_accuracy and kvalue_accuracy set the accuracies of values in real and Fourier space respectively. When an approximation must be made for some calculation, such as when to transition to Taylor approximation as small $r$ or $k$, the error in the approximation is constrained to be no more than one of these values times the total flux. The default for each is $1 . e-5$.

- realspace_relerr and realspace_abserr set the relative and absolute error tolerances for real-space convolution. The estimated integration error for the flux value in each pixel is constrained to be less than either realspace_relerr times its own flux or realspace_abserr times the object's total flux. The default values are $1 . e-4$ and $1 . e-6$ respectively.

- shoot_accuracy sets the relative accuracy on the total flux when photon shooting. When the photonshooting algorithm needs to make approximations, such as how high in radius it must sample the radial profile, the resulting fractional error in the flux is limited to shoot_accuracy. The default value is $1 . e-5$.

There are several less important parameters that can also be set similarly, but the above parameters are the most relevant for most use cases. All the GALSIM objects described in $\S 3$ have an optional parameter gsparams that can set any number of these parameters to a non-default value when initializing the object.

\subsection{Representing realistic galaxies}

GALSIM can carry out a process ('reconvolution') to render an image of a real galaxy observed at high resolution (with the $H S T)$, removing the $H S T$ PSF, with an applied lensing shear and/or magnification, as it would be observed with a lowerresolution telescope. Reconvolution was mentioned in Kaiser (2000) and implemented in Mandelbaum et al. (2012) in the SHERA (SHEar Reconvolution Analysis) software ${ }^{27}$.

\footnotetext{
${ }^{27}$ http://www.astro.princeton.edu/ rmandelb/shera/ shera.html
}

Reconvolution is possible when the target band limit $k_{\text {max,targ }}$ relates to the original $H S T$ band limit $k_{\max , \mathrm{HST}}$ via

$$
k_{\max , \operatorname{targ}}<\left(1-\sqrt{\kappa^{2}+\gamma^{2}}\right) k_{\max , \mathrm{HST}} .
$$

For weak shears and convergences, this condition is easily satisfied by all upcoming lensing surveys, even those from space.

In GALSIM, the RealGalaxy class represents the HST galaxy deconvolved by its original PSF. This can then be transformed (sheared, etc.) and convolved by whatever final PSF is desired. Observations of galaxies from the HST COSMOS survey (described in Koekemoer et al., 2007), which form the basis of the Real Gal axy class, are available for download from the GALSIM repository $^{28}$. Padding the input postage stamp images is necessary, for the reasons described in $\$ 6.2 .4$. We carry out noise padding on the fly, with a noise field corresponding to that in the rest of the postage stamp (unlike SHERA, which required padded images as inputs). The amount of padding was extensively tested during the numerical validation of the GALSIM software, and results for the default (strongly recommended) choice are described in $\$ 9.2$.

The implementation is updated compared to that in SHERA in several ways. First, SHERA only implemented a cubic interpolant, whereas GALSIM includes many interpolants that can be tuned to users' needs (c.f. §3.3.3). Second, GALSIM fully deconvolves the original HST PSF. SHERA uses a technique called pseudo-deconvolution, only deconvolving the HST PSF on scales accessible in the final low-resolution image. In practice, the difference between pseudo-deconvolution and deconvolution is irrelevant, and what matters is the ability to render sheared galaxies very accurately (see $\S 9$ ). Finally, the original HST images typically contain correlated noise, and further correlations are introduced by the shearing and subsequent convolution. In \$7.5 we describe how GALSIM (unlike SHERA) can model this correlated noise throughout the reconvolution process and then whiten the final rendered image so that it contains only uncorrelated Gaussian noise.

\section{Noise models}

Noise is a feature of all real imaging data, due to finite numbers of photons arriving at each detector, thermal noise and electronic noise within the detector and readout, and other lossy processes. GALSIM therefore provides a number of stochastic noise models that can be applied to simulated images.

It is worth noting that images rendered by photon shooting (see \$6.3) already contain noise: photon counts are drawn from a Poisson distribution of mean equal to the expected flux at each pixel. But there may still be occasions when it is desirable to add further noise, such as to simulate a shallower image or to add detector read noise. Images rendered by DFT (\$6.2) need noise to be added to be made realistic.

\footnotetext{
${ }^{28}$ https://github.com/GalSim-developers/Galsim/ wiki/RealGalaxy20Data
} 


\subsection{Random deviates}

Underlying all the noise models in GALSIM are implementations of random deviates that sample from a range of probability distributions. These include the uniform distribution $\mathcal{U}(0,1)$ (implemented by the UniformDeviate class), the Gaussian/normal distribution $\mathcal{N}\left(\mu, \sigma^{2}\right)$ (GaussianDeviate), and the Poisson distribution Pois $(\lambda)$ (PoissonDeviate).

These fundamentally important distributions form the basis of noise models in GALSIM, but they are also quite useful for generating random variates for various galaxy or PSF parameters in large simulations. Additionally, we include some other distributions that are more useful in this latter context rather than for noise models: the binomial distribution, the Gamma distribution, the Weibull distribution (a generalization of the exponential and Rayleigh distributions) and the $\chi^{2}$ distribution (see, e.g., Johnson et al., 2005).

Finally, GALSIM also supports sampling from an arbitrary probability distribution function, supplied by the user in the form of either a lookup table and interpolating function, or a callable Python function with a specified min and max value range. This is the implemented by the DistDeviate class.

\subsection{Uncorrelated noise models}

The majority of noise models that GALSIM implements generate uncorrelated noise, i.e. noise that takes a statistically independent value from pixel to pixel. The simplest is the GaussianNoise class, which adds values drawn from $\mathcal{N}\left(0, \sigma^{2}\right)$ to an image. The noise is thus spatially stationary, i.e. having a constant variance throughout the image, as well as being uncorrelated.

The PoissonNoise class adds Poisson noise Pois $\left(\lambda_{i}\right)$ to the $i$-th pixel of the image, where $\lambda_{i}$ corresponds to the mean number of counts in that pixel (an optional sky level can be supplied when simulating sky-subtracted images). The PoissonNoise model is not stationary, since the variance varies across the image according to the different pixel fluxes.

The CCDNoise class provides a good approximation to the noise found in normal CCD images. The noise model has two components: Poisson noise corresponding to the expected photon counts $\lambda_{i}$ in each pixel (again with optional extra sky level) and stationary Gaussian read noise $\mathcal{N}\left(0, \sigma^{2}\right)$. It is also possible to specify a gain value in photons/ADU (analog-to-digital units) ${ }^{29}$, in which case the Poisson noise applies to the photon counts, but the image gets values in ADU.

The VariableGaussianNoise class implements a nonstationary Gaussian noise model, adding noise drawn from $\mathcal{N}\left(0, \sigma_{i}^{2}\right)$, using a different variance for each pixel $i$ in the image.

Finally, any random deviate from $\$ 7.1$ can be used as the basis for a simple GALSIM noise model, using the DeviateNoise class, which draws from the supplied random deviate independently for each pixel to add noise to the image.

\footnotetext{
${ }^{29}$ Normally the gain is given in e-/ADU, but in GALSIM we combine the effect of the gain with the quantum efficiency, given in e-/photon
}

\subsection{Correlated noise}

Astronomical images may contain noise that is spatially correlated. This can occur at low levels due to detector crosstalk (e.g. Moore et al., 2004; Antilogus et al., 2014) or the use of pixel-based corrections for charge transfer inefficiency (CTI, Massey et al. 2010), and it can be strikingly seen in images that have been created through the coaddition of two or more dithered input images.

Drizzled HST images (Fruchter \& Hook, 2002; Koekemoer et al., 2003) often have correlated noise due to the assignment of flux from single input pixels to more than one output pixel. The drizzled HST COSMOS survey images (see Koekemoer et al., 2007) used as the basis for empirical galaxy models described in $\$ 6.5$ contain significant inter-pixel noise correlations. For faint objects, such as the galaxies typically of interest for weak lensing, such noise is well approximated as a set of correlated Gaussian random variables.

\subsubsection{Statistics of correlated Gaussian noise}

The statistical properties of correlated Gaussian noise are fully described by a covariance matrix. If we denote a noise field as $\eta$, we may define a discrete pixel-noise autocorrelation function

$$
\xi_{\text {noise }}[n, m]=\left\langle\eta[i, j] \eta\left[i^{\prime}, j^{\prime}\right]\right\rangle_{i^{\prime}-i=n, j^{\prime}-j=m},
$$

where the angle brackets indicate the average over all pairs of pixel locations $[i, j]$ and $\left[i^{\prime}, j^{\prime}\right]$ for which $i^{\prime}-i=n$ and $j^{\prime}-j=m$. (Here we use square brackets for functions with discrete, integer input variables.) Therefore $n, m$ denote the integer separation between pixels in the positive $x$ and $y$ directions, respectively. All physical $\xi_{\text {noise }}[n, m]$ functions have two-fold rotational symmetry, so that $\xi_{\text {noise }}[-n,-m]=\xi_{\text {noise }}[n, m]$, and are peaked at $n=m=0$. If the noise is stationary, i.e. its variance and covariances do not depend upon location in the image, then $\xi_{\text {noise }}[n, m]$ fully determines the covariance matrix for all pairs of pixels in an image.

For any physical, discrete pixel-noise correlation function $\xi_{\text {noise }}[n, m]$ there is a positive, real-valued noise power spectrum $P_{\text {noise }}[p, q]$ that is its Fourier counterpart (i.e. the two are related by a DFT). We use $p, q$ to label array locations in Fourier space, and $n, m$ in real space.

The function $P_{\text {noise }}[p, q]$ can be used to generate random noise as a Gaussian field. We may construct an array $\widetilde{\eta}[p, q]$ as

$$
\widetilde{\eta}[p, q]=\sqrt{\frac{P_{\text {noise }}[p, q] N}{2}}\{\mathcal{N}(0,1)+\mathrm{i} \mathcal{N}(0,1)\}
$$

where samples from the standard Gaussian distribution $\mathcal{N}(0,1)$ are drawn independently at each location $p, q$ (subject to the condition that $\tilde{\eta}[p, q]$ is Hermitian, see below), and $N$ is the total array dimension in either direction (we assume square arrays for simplicity). The inverse DFT, as defined in equation (A.17), is then applied to generate a real-valued noise field realization in real space $\eta[n, m]$. The enforced Hermitian symmetry of $\widetilde{\eta}[p, q]$ is exploited to enable the use of efficient inverse-real DFT algorithms. It can be seen that the resultant $\eta[n, m]$ will have the required discrete correlation function $\xi_{\text {noise }}[n, m]$. 


\subsubsection{Representing correlated Gaussian noise}

Stationary correlated noise is modelled and generated in GALSIM by the CorrelatedNoise class. Internally the noise correlation function $\xi_{\text {noise }}[n, m]$ is represented as a $2 \mathrm{D}$ distribution using the same routines used to describe astronomical objects, described in $\S 3$. Using these to calculate the noise power spectrum, and then applying the expression in equation (67) followed by an inverse DFT, the CorrelatedNoise class can be used to generate a Gaussian random field realization with any physical power spectrum or correlation function.

Because the CorrelatedNoise class internally uses a regular GALSIM surface brightness profile to describe the correlation function, it is easy to effect the transformation, as in $\S 3.4$, since the noise is transformed in the same manner. Similarly, multiple noise fields may be summed (as will be required in $\$ 7.5$ ), since the resultant $\xi_{\text {noise }}$ is given by the sum of the individual noise correlation functions.

The effect of convolution of a noise model by some kernel is not quite the same as what happens to surface brightness. Instead, $\xi_{\text {noise }}$ should be convolved by the autocorrelation of the kernel. The ability to describe transformations, combinations and convolutions of noise is valuable, as will be discussed in $\S 7.5$.

\subsubsection{Describing discrete correlation functions in $\mathbb{R}^{2}$}

The description of the discrete correlation function $\xi_{\text {noise }}[n, m]$ as a distribution in $\mathbb{R}^{2}$ is worthy of some discussion. As will be seen in $\$ 7.5$ it is important to be able to take into account the finite size of pixels in images that may contain correlated noise, particularly when these images are being used to form galaxy models that will ultimately be rendered onto a pixel grid of a different scale. We therefore wish to represent $\xi_{\text {noise }}[n, m]$ as a function of continuous spatial variables, $\xi_{\text {noise }}(x, y)$ for which we require

$$
\xi_{\text {noise }}(n s, m s)=\xi_{\text {noise }}[n, m],
$$

where $s$ is the pixel scale.

Let us consider an image containing noise. In the absence of any smoothing of the image, it is described mathematically as an array of delta function samples (located at the pixel centres) convolved by the pixel response. This could also be considered the formally correct model to use for $\xi_{\text {noise }}(x, y)$ : its values, like those in an image, should change discontinuously across the boundaries between pixels as separation increases continuously.

However, this description of $\xi_{\text {noise }}(x, y)$ presents difficulties when performing certain of the operations described in $\$ 7.3 .2$, e.g. convolutions, or transformations followed by a convolution. The presence of sharp discontinuities at every pixel-associated boundary makes the necessary Fourier space representation of $\xi_{\text {noise }}(x, y)$ extremely non-compact, and prohibitive in terms of memory and computing resources.

Therefore, to reduce the computational costs of handling Gaussian correlated noise to tolerable levels, GALSIM adopts bilinear interpolation between the sampled values $\xi_{\text {noise }}(n s, m s)$ to define the continuous-valued function $\xi_{\text {noise }}(x, y)$ within the correlatedNoise class.

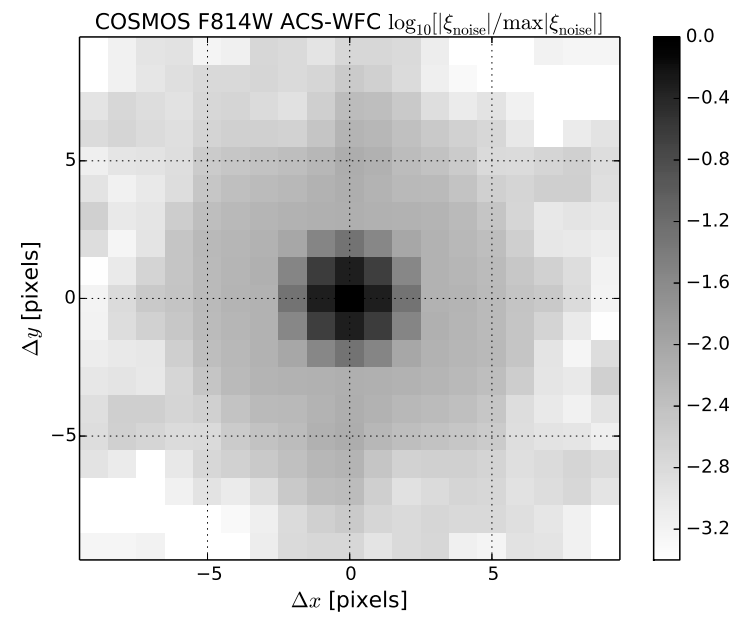

Figure 1: Illustration of the GALSIM estimate of the discrete pixel noise correlation function $\xi_{\text {noise }}$ in the 0.03 arcsec/pixel, unrotated COSMOS F814W science images of Leauthaud et al. (2007), made by averaging estimates from blank sky fields as described in $\$ 7.4$. The plot depicts the log of the correlation function normalized so that $\xi_{\text {noise }}[0,0]=1$ for clarity, and shows only the central region corresponding to small separations $\Delta x, \Delta y$, which dominate covariances.

In practice, the impact of this efficiency-driven approximation may often be small. The primary use case for the representation of correlated noise within GALSIM is in handling noise in models derived from direct observations of galaxies (see $\$ 6.5$ and $\$ 7.5$ ). In these applications, both the applied PSF and the pixel scale on which the final image will be rendered are typically significantly larger than the pixel scale of the input images. The use of bilinear interpolation represents a small additional convolution kernel in these cases.

\subsection{COSMOS noise}

The HST COSMOS galaxy images made available for download with GALSIM for use in the RealGalaxy class (see Koekemoer et al., 2007; Leauthaud et al., 2007; Mandelbaum et al., 2012) contain noise that is spatially correlated due to drizzling. To construct a model for this correlated noise, we estimated the discrete pixel noise correlation function in $\sim 100$ rectangular regions selected from empty sky in the public, unrotated, ACS-WFC F814W science images ( 0.03 arcsec/pixel) from COSMOS (described in detail by Koekemoer et al., 2007; Leauthaud et al., 2007).

Each rectangular region varied in size and dimensions, being chosen so as not to include any detected objects, but were typically square regions $\sim 50-200$ pixels along a side. The mean noise correlation function in these images (calculated by summing over each individual estimate, see \$7.3.2) provides an estimate of the mean noise correlation function in COSMOS F814W galaxy images used by GALSIM as the basis for galaxy models. The resulting estimate of $\xi_{\text {noise }}$ is depicted in Fig. 1.

This model is included with the GALSIM software for general use in handling images that contain noise like those in the COSMOS F814W images, and is accessible via the getCOSMOSNoise ( ) convenience function. 


\subsection{Noise whitening}

All images of galaxies from telescope observations will contain noise. As pointed out by Mandelbaum et al. (2012), this noise will become sheared and correlated (in an anisotropic way) by the action of the reconvolution algorithm described in $\S 6.5$, which can result in a significant systematic offset in the results from galaxy shear simulations. Mandelbaum et al. (2012) found that the presence of correlated noise caused $\sim 1 \%$ level errors in the determination of calibration biases for weak lensing shear for high- $S / N$ galaxies; the effect is worse at lower $S / N$, and cannot be ignored for high-precision shear simulations.

GALSIM addresses the presence of correlated noise in simulated images, such as the sheared, convolved noise fields created by the reconvolution algorithm, through a process referred to as "noise whitening". This technique adds further noise to images, with a correlation function chosen to make the combination of the two noise fields approximately uncorrelated and stationary (aka "white").

We consider an image containing correlated Gaussian noise that we label as $\eta$. We assume $\langle\eta\rangle=0$ and we $\operatorname{model} \eta$ as stationary across the image so that its covariance matrix is fully described by its correlation function $\xi_{\text {noise }}[n, m]$ or power spectrum $P_{\text {noise }}[p, q]$. We add additional whitening noise which we label $\eta_{\text {whitening }}$, giving a combined noise field

$$
\eta_{\text {total }}=\eta+\eta_{\text {whitening }}
$$

The statistics of $\eta_{\text {whitening }}$ are also determined by its power spectrum, $P_{\text {whitening }}[p, q]$, and $\eta_{\text {whitening }}$ will be physically realizable provided that

$$
P_{\text {whitening }}[p, q] \geq 0
$$

for all $p, q$. The power spectrum of the combined noise field is simply

$$
P_{\text {total }}=P_{\text {noise }}+P_{\text {whitening }} \text {. }
$$

We may choose $P_{\text {whitening }}$ so that $\eta_{\text {total }}$ is uncorrelated by requiring $P_{\text {total }}$ to be a constant (a flat power spectrum corresponds to a delta function correlation function, which is white noise). This, and the requirement $P_{\text {whitening }} \geq 0$, is satisfied by choosing

$$
P_{\text {whitening }}[p, q]=\max _{p, q}\left\{P_{\text {noise }}[p, q]\right\}-P_{\text {noise }}[p, q]
$$

In practice, GALSIM adds a small amount of additional variance so that $P_{\text {whitening }}>0$, defining

$$
\begin{gathered}
P_{\text {whitening }}[p, q]=(1+\epsilon) \times \max _{p, q}\left\{P_{\text {noise }}[p, q]\right\} \\
-P_{\text {noise }}[p, q]
\end{gathered}
$$

where $\epsilon=0.05$ by default. The whitening noise $\eta_{\text {whitening }}$ can then be added following the prescription described in \$7.3.1, using the expression for $P_{\text {whitening }}$ above. At a relatively small fractional cost in additional variance, the 'headroom' parameter $\epsilon$ effectively guarantees $P_{\text {whitening }}[p, q] \geq 0$. Without $\epsilon$ this important condition might not be met, due to numerical rounding and approximations in the description of $\xi[n, m]$ (see $\$ 7.3 .3$ ).
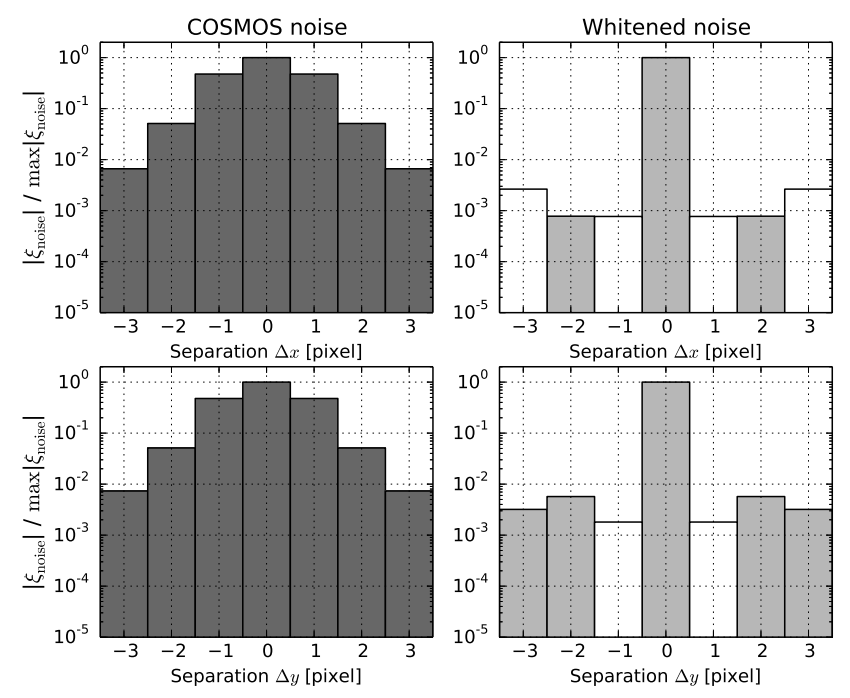

Figure 2: Left panels: cross section through the discrete pixel noise correlation function $\xi_{\text {noise }}$ for the COSMOS F814W blank sky fields described in $\$ 7.4$ and shown in Fig. 1, normalized to unit zero-lag variance; the upper left panel shows COSMOS noise correlations in the direction $\Delta y=0$, and the lower left panel shows COSMOS noise correlations in the direction $\Delta x=0$. Right panels: cross section through the normalized discrete pixel noise correlation functions for a single $\sim 400$ pixel $\times 300$ pixel patch of blank sky, taken from COSMOS, to which further whitening noise has been added using the $\$ 7.4$ model for $\xi_{\text {noise }}$, using the procedure described in $§ 7.5$. As for the left panels, the upper right and lower right panels show correlations along the directions $\Delta y=0$ and $\Delta x=0$, respectively. Unfilled bars represent negative correlation function values.

Fig. 2 shows cross sections through $\xi_{\text {total }}[n, m]$ from a single $\sim 400 \times 300$ pixel patch of blank sky taken from COSMOS F814W ACS-WFC images, to which further whitening noise has been added. $P_{\text {whitening }}$ was calculated using the model for $\xi_{\text {noise }}$ (and thus $P_{\text {noise }}$ ) described in $\$ 7.4$ along with equation (73). Here GALSIM noise whitening has lowered interpixel covariances to $\sim 10^{-2}-10^{-3}$ of the zero-lag variance in the whitened image.

We have tested that applying this whitening procedure to COSMOS noise fields that have been sheared and convolved (like those produced by the reconvolution algorithm of $\$ 6.5$ ) similarly reduce the noise correlations by $2-3$ orders of magnitude, making them statistically undetectable in all but the largest simulated images.

The price paid for noise whitening is additional variance in the output image. The noise added in equation (69) is an additional stochastic degradation of an image. For the COSMOS F814W noise field whitened in Fig. 2, the final noise field therefore has a variance that is greater than the variance in the original images. However, the correlated noise in a COSMOS image itself results in a lower effective $S / N$ for estimates of object properties such as flux, or shape, relative to the case of an image with the same variance but with pure white noise (for a discussion of these effects, which depend on object size and the properties of the correlated noise, see, e.g., Casertano et al., 2000; Leauthaud et al., 2007). The effect of additional whitening of the noise in such an image, therefore, reduces the $S / N$ of estimates of object properties by less than implied by the ra- 
tio of pre- and post-whitening image variances. The amount of information lost in whitening will depend on the property in question, the object profile, and the noise correlations in the original image.

In the case of noise fields that have been sheared, convolved by a PSF, and then rendered on an image with a larger pixel scale (as described in $\$ 6.5$ ), the noise added in whitening depends not only on the original noise correlation function, but also on the applied shear, PSF, and final pixel scale. Interestingly, it is found to be typically substantially lower in overall variance than in the case of whitening raw COSMOS noise at native resolution (the case described above and shown in Fig. 2).

On investigation, this was found to be due to a combination of effects. The shear and convolution both increase the noise correlations at large scales. However, the final image rendering typically has a pixel scale $s$ significantly larger than the 0.03 arcsec/pixel of the COSMOS images, which means that the values of the correlation function at integer multiples of $s$ in separation are often smaller than in the original COSMOS image. Also, convolution with a broad PSF reduces the overall noise variance considerably, being essentially a smoothing kernel. These effects combine fortuitously in practice: Mandelbaum et al. (2014) found that only a relatively modest amount of whitening noise is required to treat the sheared, correlated noise in images generated by the reconvolution algorithm of $\S 6.5$ with a ground-based target PSF and pixel scale.

In GALSIM the Image class has a method whitenNoise that applies the above whitening algorithm to a given image. This method also returns the theoretical estimate of the noise variance in the final whitened image (namely $(1+$ $\left.\epsilon) \max _{p, q}\left\{P_{\text {noise }}[p, q]\right\}\right)$. In addition, there is a method symmetrizeNoise that imposes $N$-fold symmetry (for even $N \geq$ 4) on the noise field rather than making it entirely white. Typically far less noise must be added by symmetrizeNoise compared to whitenNoise, which makes it a useful option for those applications that do not require fully white noise.

The RealGalaxy class has an attribute noise that keeps track of the estimated correlated noise in the profile. When this object is transformed or convolved, the new object will also have a noise attribute that has had the appropriate transformation done to propagate the noise in the new profile. This allows GALSIM to automatically track the effect of transformations on noise fields without much user input required. The final profile's noise attribute can then be used to whiten the rendered image. Furthermore, users can add a noise attribute themselves to any object they create and get the same behaviour.

In a larger image with many scattered, possibly overlapping galaxies, the correlated noise in the original images may differ, further complicating the task of getting uniform white noise in the final image. GALSIM handles this by drawing each galaxy on its own postage stamp image and whitening that individually. Still, the final image has a complicated patchwork of noise with different regions having different variances, but it is relatively straightforward in GALSIM to keep track of these noise levels: the configuration file interface described in $\$ 2.3$ allows this to be handled automatically. Then the VariableGaus-
sianNoise class can add a variable amount of noise to each pixel to bring the noise up to a uniform value across the entire image.

\section{Shear estimation}

GALSIM includes routines for estimation of weighted moments of galaxy shapes and for estimation of PSF-corrected shears. The code for these routines was originally introduced by Hirata \& Seljak (2003) (hereafter HS03), including an entirely new PSF correction method known as re-Gaussianization. These routines were later tested, improved, and optimized in subsequent work (Mandelbaum et al., 2005, 2012; Reyes et al., 2012). The code was developed as a free-standing $C$ package named HSM, and was released publicly under an open source license simultaneously with its incorporation into GALSIM.

The function FindAdaptiveMoments implements the "adaptive moments" method described in $\$ 2.1$ of HS03, based on Bernstein \& Jarvis (2002). It iteratively computes the moments of the best-fitting elliptical Gaussian, using the fitted elliptical Gaussian as a weight function.

The function Estimateshear implements multiple methods that were tested in HSO3:

1. The PSF correction method described in Appendix $\mathrm{C}$ of Bernstein \& Jarvis (2002) tries to analytically account for the effect of the kurtosis of the PSF (in addition to the second order moments) on the galaxy shapes.

2. The "linear" method (described in Appendix B of HSO3) is a variant of the previous method that accounts for the firstorder departure of both the PSF and galaxy from Gaussianity.

3. The "re-Gaussianization" PSF correction method described in $\$ 2.4$ of HS03 models the PSF as a Gaussian plus a residual, and subtracts from the observed image an estimate of the residual convolved with the pre-seeing galaxy. The resulting image has roughly a Gaussian PSF, for which the methods described above can be used for the final PSF correction.

4. A specific implementation of the KSB method (Kaiser et al., 1995; Luppino \& Kaiser, 1997), as described in Appendix C of HS03, is also provided.

These routines work directly on GALSIM images. The first three PSF correction methods output a measure of per-galaxy shape called a distortion, which for an ensemble of galaxies can be converted to shear estimates via a responsivity factor (cf. Bernstein \& Jarvis 2002). The outputs are clearly labeled as being distinct from per-galaxy shear estimates, such as those output by the KSB routine.

As is typical for all shape measurement algorithms, there are several tunable parameters for the above methods and for moment estimation overall (with adaptive moment estimation playing a role in all but the KSB method of PSF estimation). GALSIM therefore includes two ways of tuning these parameters. There is an HSMP arams structure that allows users to 
change parameters that affect how the submodule works overall. Other parameters are given as arguments to the specific functions.

Aside from the obvious utility of being able to operate directly on images in memory, rather than files, the versions of these routines in GALSIM come with some improvements over the free-standing $\mathrm{C}$ code. These include an intuitive and more flexible user interface; optimization of convolutions, the exp function (via reduction of the number of function calls), and Fourier transforms (using the FFTw package, Frigo \& Johnson, 2005); a clean way of including masks within images that is easily extensible to variable weight maps; and a fix for a bug that caused the original $\mathrm{C}$ code to work incorrectly if the input PSF images were not square.

\section{Numerical validation}

In this Section we describe the investigations that were undertaken to validate the accuracy of GALSIM image simulations. Although an exhaustive validation of the rendering of every combination of galaxy/PSF profiles and observing conditions is impractical, certain key aspects of GALSIM performance are shown here. Emphasis is placed on confirming that GALSIM meets the stringent requirements on image transformations for lensing shear and magnification simulation.

In particular, our metric for validating that the rendered images are sufficiently accurate is based on measurements of the size and ellipticity of the rendered profiles, calculated using the adaptive moment routines described in $\S 8$.

We define the following "STEP-like" (see Heymans et al., 2006) models for the errors in the estimates of object ellipticity $g_{1}$ and $g_{2}$ and size $\sigma$ :

$$
\begin{aligned}
& \Delta g_{i}=m_{i} g_{i}+c_{i}, \\
& \Delta \sigma=m_{\sigma} \sigma+c_{\sigma},
\end{aligned}
$$

where $i=1,2$. The method of estimating the errors $\Delta g_{i}$ and $\Delta \sigma$ varies for each of the validation tests described below, but a common component is adaptive moments estimates of rendered object shapes from images (see $\S 8$ ). We will use the formulae above when describing the nature of the errors in each test.

As discussed in Mandelbaum et al. (2014), a well-motivated target for simulations capable of testing weak lensing measurement is to demonstrate consistency at a level well within the overall requirements for shear estimation systematics set by $E u$ clid (e.g. Cropper et al., 2013; Massey et al., 2013): $m_{i} \simeq 2 \times$ $10^{-3}$ and $c_{i} \simeq 2 \times 10^{-4}$. Such values also place conservative requirements on galaxy size estimation, as the signal-to-noise expected for cosmological magnification measurements has been estimated as $\lesssim 50 \%$ relative to shear (e.g. van Waerbeke, 2010; Schmidt et al., 2012; Duncan et al., 2014).

Only if these stringent Euclid conditions are met comfortably will simulations be widely usable for testing weak lensing shear estimation, and other precision cosmological applications, in the mid-term future. For each validation test we therefore require that GALSIM can produce images showing discrepancies that are a factor of 10 or more below the Euclid requirements, i.e. $m_{x}<2 \times 10^{-4}, c_{x}<2 \times 10^{-5}$, where $x=1,2, \sigma$ corresponding to $g_{1}, g_{2}$ and $\sigma$, respectively. Our intention is to show that rendering accuracy improves with more stringent rendering parameter settings, and can be raised to meet the requirement above. GALSIM default settings for these parameters, however, are chosen to reflect a balance between speed and accuracy for general use. Although these defaults typically take values that guarantee meeting 1/10th Euclid requirements, this is not exclusively the case (see $\$ 9.3$ ).

For our tests, we use comparisons of rendering methods using adaptive moments measurements. Although there are inaccuracies inherent in such measurements in an individual sense, we take care to construct our comparisons to be sensitive only to differences in the image rendering. For example, the tests always compare noise-free or extremely low noise images, with the same underlying galaxy model. Noise bias and model bias therefore affect each test subject in the same way, and differences are due solely to how the test images were rendered.

The tests in this Section were conducted over a period of extended validation of the GALSIM software between July 2013 and the time of writing this paper. During this time period, corresponding approximately to versions 1.0 and 1.1 of GALSIM, the routines for rendering objects did not change significantly (except where modifications were found necessary to meet the validation criteria on $m_{x}$ and $c_{x}$ defined above).

\subsection{Equivalence of DFT rendering and photon shooting}

One of the principal advantages of the photon shooting method (see $\S 6.3$ ) is that the implementations of the various transformations described in $\$ 3.4$ are very simple. Photons are just moved from their original position to a new position. Convolutions are similarly straightforward. On the other hand, DFT rendering (see §6.2) needs to deal with issues such as band limiting and aliasing due to folding (cf. §6.2.1).

Thus a powerful test of the accuracy of our DFT implementation is that the the two rendering methods give equivalent results in terms of measured sizes and shapes of the rendered objects. An unlikely conspiracy of complementing errors on both sides would be required for this test to yield false positive results.

Of all the objects in Table 3, Sérsic profiles are the most numerically challenging to render using Fourier methods. Especially for $n \gtrsim 3$, the profiles are extremely cuspy in the centre and have very broad wings, which means that they require a large dynamic range of $k$ values when performing the DFT. They therefore provide a good test of our choices for parameters such as folding_threshold and maxk_threshold (see §6.4) as well as general validation of the DFT implementation strategies.

For our test, we built Sersic objects with Sérsic indices in the range $1.5 \leq n \leq 6.2$. The half-light radii and intrinsic ellipticities $\left|g^{(s)}\right|$ were drawn from a distribution that matches observed COSMOS galaxies, as described in Mandelbaum et al. (2014). The galaxies were then rotated to a random orientation, convolved with a COSMOS-like PSF (a circular Airy profile), and then rendered onto an image via both DFT and photon shooting. 
The error estimates were taken to be the difference between the adaptive moments shape and size estimates from the two images:

$$
\begin{aligned}
\Delta g_{i} & =g_{i, \mathrm{DFT}}-g_{i, \mathrm{phot}} \\
\Delta \sigma & =\sigma_{\mathrm{DFT}}-\sigma_{\mathrm{phot}}
\end{aligned}
$$

For each galaxy model, we made multiple trial photon shooting images, each with very high $S / N$ to avoid noise biases $\left(10^{7}\right.$ photons shot per trial image). The mean and standard error of $\Delta g_{i}$ and $\Delta \sigma$ from these trials were used to estimate values and uncertainties for $m_{x, \mathrm{DFT}}$ and $c_{x, \mathrm{DFT}}$ using standard linear regression.

Differences between shape and size estimates are illustrated in Fig. 3, for $n=1.5$ and $n=6.2$. Fig. 4 shows derived estimates of $m_{x, \mathrm{DFT}}$ for these and other Sérsic indices tested. Tolerances are met on $m$-type biases. It was found that $c$-type additive biases were consistent with zero for all $n$ indices.

Fig. 5 shows results from a high-precision investigation of $m_{x, \text { DFT }}$ as a function of GSParams parameters (see $\$ 6.4$ ), using a randomly selected sample of 270 galaxies from COSMOS at each parameter value and large numbers of photons. Each galaxy was generated in an 8-fold ring test configuration (Nakajima \& Bernstein, 2007) to further reduce statistical uncertainty. The plot in Fig. 5 shows the impact of increasing the folding_threshold parameter: as expected, the rendering agreement decreases as folding_threshold increases, and the representation of object size is most affected. Analogous results were achieved for many of the parameters discussed in $\S 6.4$, and the default GSParams parameters were found to give conservatively good performance in all tests.

\subsection{Accuracy of transformed interpolated images}

Interpolated images pose unique challenges for DFT rendering. As we discussed in $\$ 6.2 .4$, details such as the choice of the Fourier-space interpolant and how much padding to use around the original image significantly affect the accuracy of the rendering. We thus need to validate that these choices can be varied to produce sufficiently accurate results.

For this test, we randomly selected a sample 6000 Sérsic models from the catalogue COSMOS galaxy fits described in Mandelbaum et al. (2014). For each galaxy in the sample, we constructed a Sersic object using the best-fitting parameters in the catalogue, which was then convolved by a COSMOSlike PSF (a circular Airy profile). This profile was drawn onto an image with 0.03 arcsec resolution (matching the COSMOS images). That image was then used to construct an Interpolated Image profile.

For each object in the sample we therefore had the same profile modelled both as an analytic convolution and as an interpolated image. Small shears were then applied to both models and the resulting profiles were drawn onto images using the DFT rendering algorithm, although without including the integration by a pixel. Each profile was convolved by a tiny Gaussian to force GALSIM to use the DFT rendering method (as direct rendering would otherwise be used when omitting the pixel convolution).
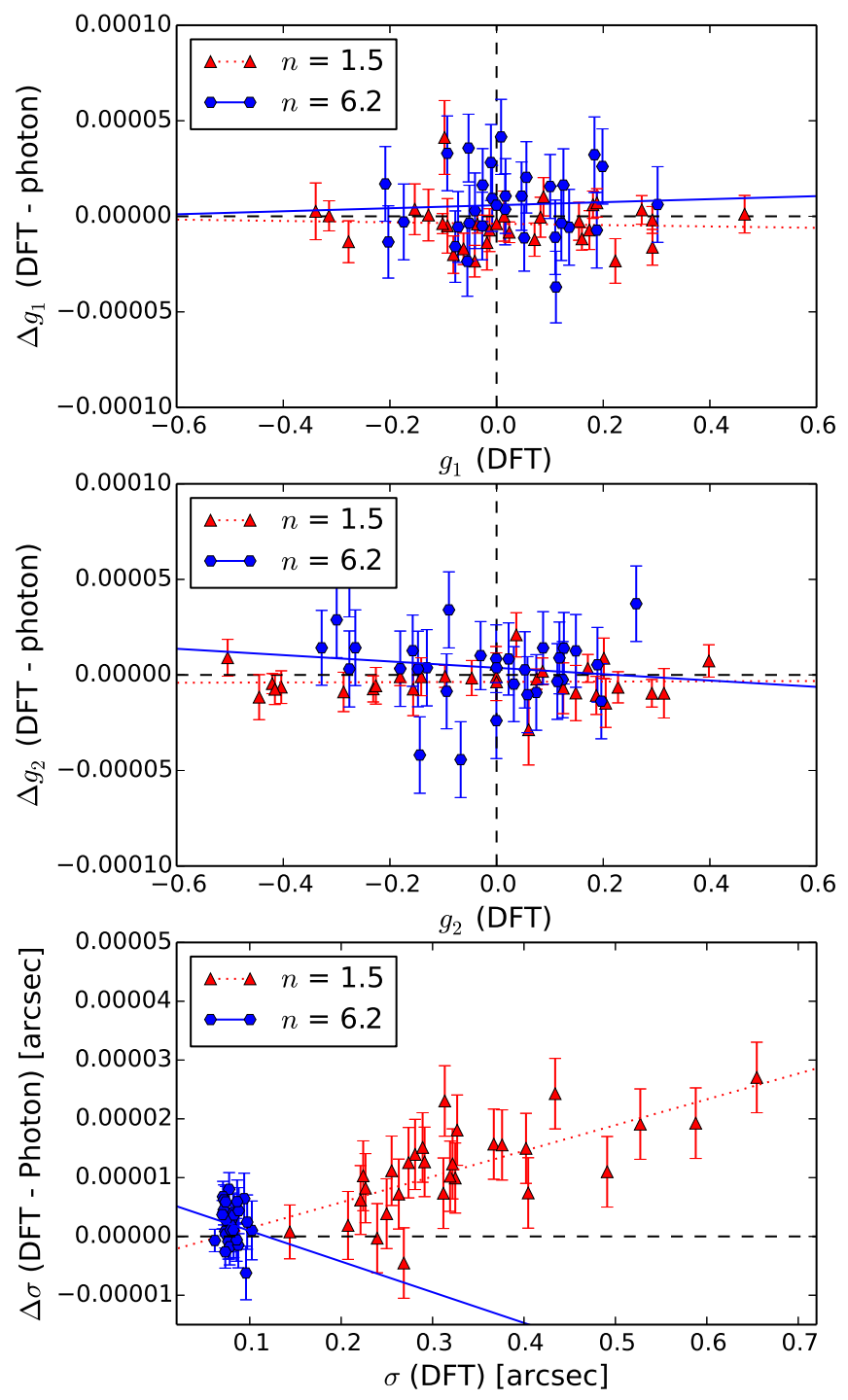

Figure 3: Difference between measured shears (upper panel: $g_{1}$; central panel: $g_{2}$; lower panel: $\sigma$ ) for Sérsic profiles simulated using the photon-shooting and DFT rendering methods, plotted against the (shot noise free) shear and size measured from the DFT image. Results are shown for 30 galaxies with realistic size and shape distribution, and Sérsic index values $n=1.5,6.2$. (Note that the 'peakiness' of the high- $n$ profiles results in their low $\sigma$ estimates. The two samples share the same distributions of half light radii.) The best-fitting lines are shown, and estimates of the slopes $m_{x, \mathrm{DFT}}$ for these and other values of $n$ are plotted in Fig. 4. 


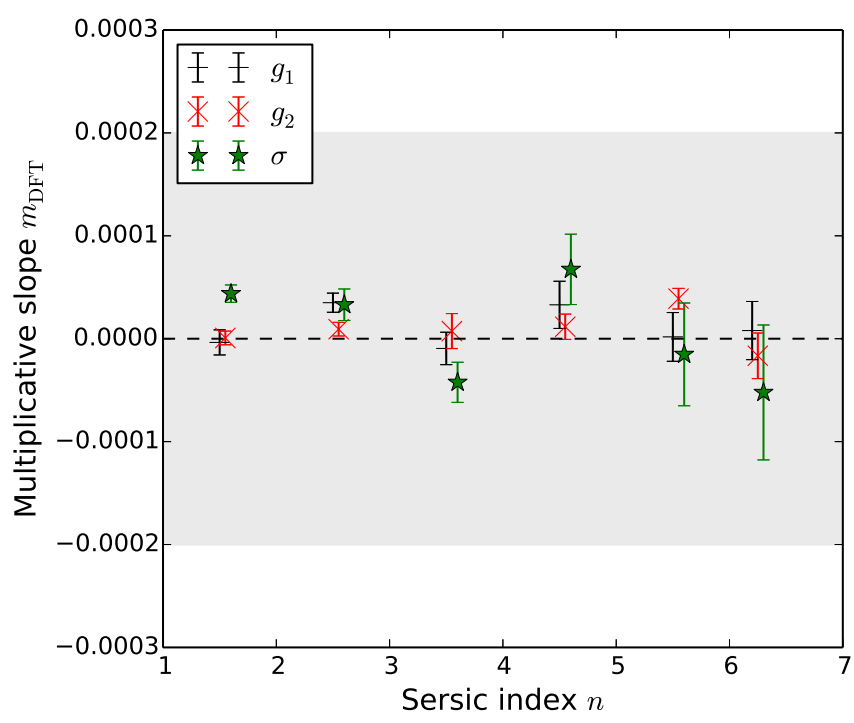

Figure 4: Estimates of $m_{1, \mathrm{DFT}}, m_{2, \mathrm{DFT}}$ and $m_{\sigma, \mathrm{DFT}}$, corresponding to renderinginduced discrepancies in ellipticity $g_{1}, g_{2}$ and size $\sigma$, respectively, as a function of Sérsic index $n$. These slope parameters are defined by equations (74) \& (75) for the differences between measurements from DFT and photon-shootingrendered images of Sérsic profiles. The shaded region shows the target for GALSIM based on not exceeding one tenth of weak lensing accuracy requirements for Stage IV surveys such as Euclid (see §9).

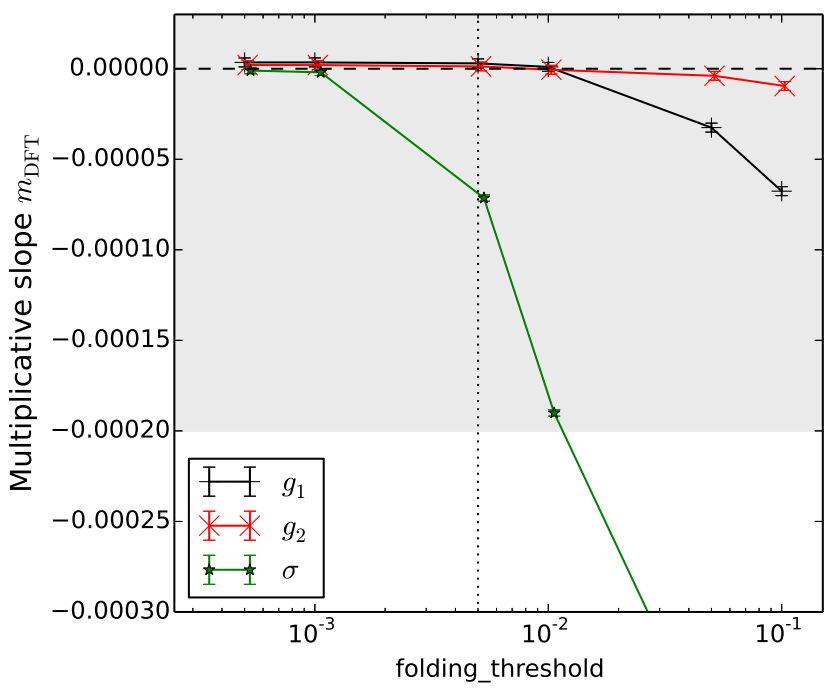

Figure 5: Estimates of $m_{1, \mathrm{DFT}}, m_{2, \mathrm{DFT}}$ and $m_{\sigma, \mathrm{DFT}}$, corresponding to renderinginduced discrepancies in ellipticity $g_{1}, g_{2}$ and size $\sigma$, respectively, as a function of the GSParams parameter folding_threshold. Each point shows the average from the randomly-selected sample of 270 unmodified COSMOS galaxy models described in \$9.1. The rendering parameter folding_threshold is described in $\$ 6.4$ and takes a default value of $5 \times 10^{-3}$, indicated by the dotted line. As for Fig. 4 these parameters are defined by the model of equations (74) \& (75). The shaded region shows the target for GALSIM based on not exceeding one tenth of weak lensing accuracy requirements for Stage IV surveys (see $\$ 9$ ).
Adaptive moments estimates of the change in ellipticity were seen to be consistent between the parametric model and interpolated image at better than 1/10th Euclid requirements, for all real and Fourier space interpolants of higher order than bilinear. Crucially, both were consistent with the known applied shear. Results for changes in object size were similar. These tests validated the Interpolated Image handling of transformations for simple input images derived from parametric profiles (such as a Sérsic galaxy convolved with a COSMOS PSF).

The test was then extended to the more complicated case of RealGalaxy profiles. Once again, adaptive moments estimates of the object ellipticity were made before and after applying a shear. This was compared to the expected shear, calculable given an adaptive moments estimate of the intrinsic ellipticity prior to shearing, and the known applied shear. The RealGalaxy profile was again convolved with a tiny Gaussian PSF prior to rendering (see above), but in this case additional whitening noise was applied to verify that the presence of sheared, correlated noise in the output was being appropriately handled.

This test was repeated for the same sample of $6000 \mathrm{COS}$ MOS galaxy images. Fig. 6 shows estimates of $m_{i \text {,interp for a }}$ range of Fourier space interpolants, and for two values of the pad_factor parameter, which specifies how large a region around the original image to pad with zeroes. A larger value for pad_factor produces more accurate results, as shown in Fig. 6, but is accompanied by large increases in computation time.

We performed many similar tests to this for a range of transformations (in various combinations) and input parameters. In all tests performed, setting pad_factor $=4$ and using the two-dimensional quintic interpolant gave results that satisfied $m_{i, \text { interp }}<2 \times 10^{-4}, c_{i, \text { interp }}<2 \times 10^{-5}$. These results justify the choice of these as the defaults in GALSIM. Values of $c_{i \text {,interp }}$ were found to be extremely small in general.

In addition to verifying direct shear response, we also checked for leakage between shear components, i.e., that applying shear in one component does not result in an incorrect level of shear in the other component. The leakage was found to be consistent with zero and comfortably within requirements for pad_factor values of 4 and 6, for all interpolants tested.

The only RealGalaxy test that did not pass our requirement was when we simultaneously simulated shears in both components and a magnification. In this case we found $m_{\sigma \text {,interp }} \sim(4 \pm 0.4) \times 10^{-4}$, largely irrespective of the choice of interpolant. The source of this bias, which is larger than the target $2 \times 10^{-4}$, has not yet been identified. However, as magnification is a cosmological probe that is typically noisier than shear, this degree of discrepancy is likely to be tolerable for simulations of magnification in Stage IV survey data.

\subsection{Accuracy of reconvolution}

As a final demonstration of GALSIM's high precision operation, we tested that we can accurately apply the reconvolution algorithm of $\S 6.5$ (Mandelbaum et al., 2012). The aim is to represent the appearance of a test object following an applied shear $g_{\text {applied }}$, when viewed at lower resolution. 

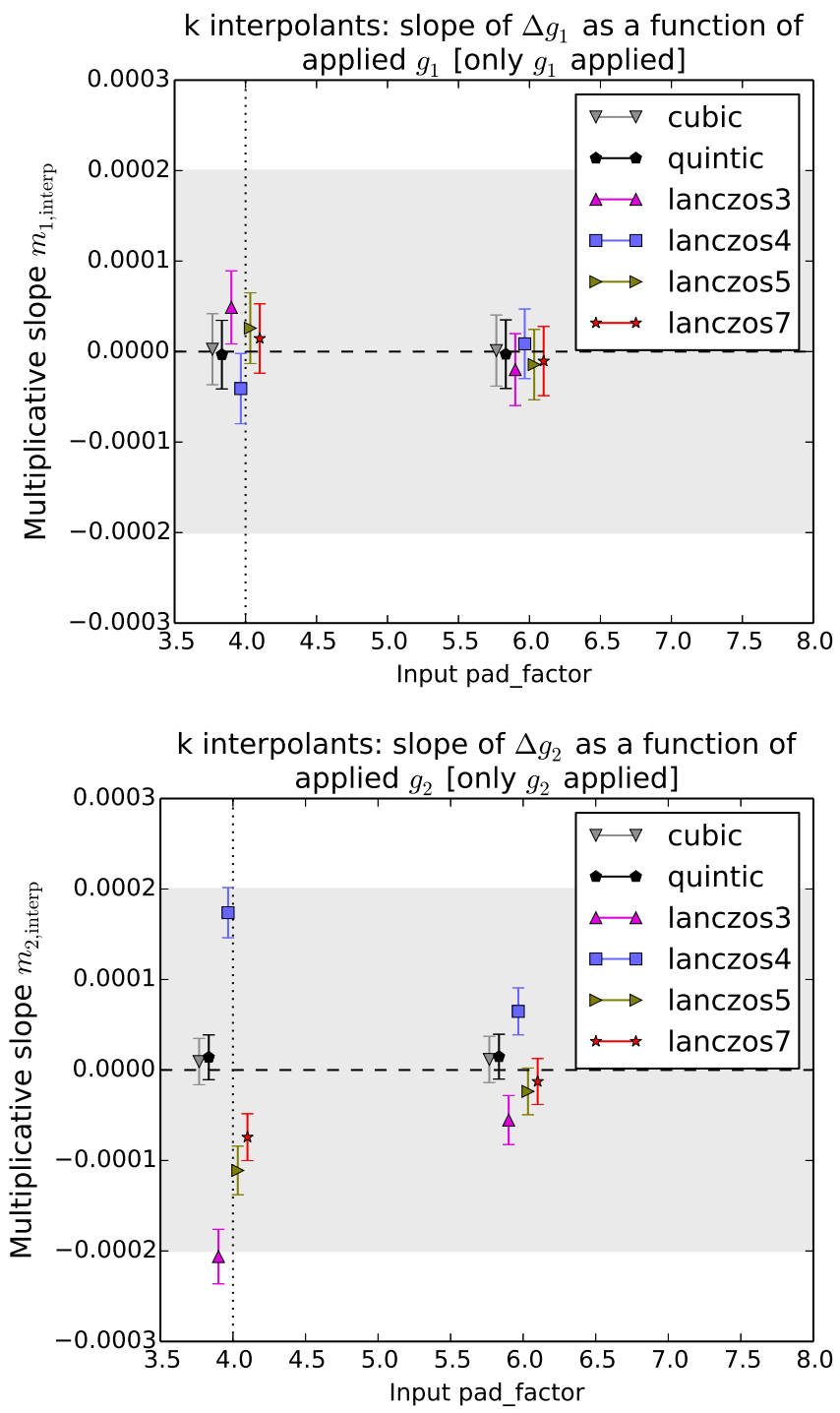

Figure 6: Multiplicative bias $m_{i, \text { interp }}$ in the relationship between an applied shear and an adaptive moments estimate of the observed shear for RealGal axy objects, for various Fourier space interpolants, e.g. cubic, quintic etc., and the padding factor (default $=4$, indicated by the dotted line). These results include the handling of correlated noise via the noise whitening procedure described in $\$ 7.5$. The shaded region shows the target for GALSIM based on not exceeding one tenth of weak lensing accuracy requirements for Stage IV surveys (see $\$ 9$ ).
This test was carried out using Sersic profiles convolved by a known COSMOS-like PSF (a circular Airy profile), rendered at high resolution (0.03 arcsec/pixel). These images, along with images of the PSF, were then used as inputs to initialize RealGalaxy objects, mimicking the use of real COSMOS galaxy images. In the usual manner these objects were sheared and reconvolved by a broader (ground-based or Stage IV space-based survey) PSF, then rendered at lower resolution.

Because of the use of an underlying parametric Sérsic profile, the rendering of which has been validated in $\$ 9.1$, we can also render the convolved, sheared object directly at lower resolution to provide a reference for comparison. We quantify any error in the effectively applied shear due to the reconvolution process as $m_{i \text {,reconv }}$ and $c_{i \text {,reconv }}$, defined according to equation (74).

The test was done for 200 profiles whose parameters were selected from the real COSMOS galaxy catalogue described in Mandelbaum et al. (2014), using random galaxy rotations in an 8-fold ring test configuration (Nakajima \& Bernstein, 2007).

Since galaxies with different light profiles might be more or less difficult to accurately render using reconvolution, we must consider not only the mean values of $m$ and $c$, but also investigate their ranges, which could identify galaxy types for which the method fails to work sufficiently accurately even if it is successful for most galaxies.

Fig. 7 shows $m_{i, \text { reconv }}$ as a function of the folding_threshold parameter described in \$6.4. Near the GALSIM default value of $5 \times 10^{-3}$, our requirement $m_{i \text {,reconv }}<$ $2 \times 10^{-4}$ is met comfortably in the ensemble average. Across the sample of 200 COSMOS galaxies a small fraction $(3 / 200)$ exceeded our requirement for the default folding_threshold value for $m_{2 \text {,reconv }}$. However, we do not believe that this represents enough of a concern to change the default GSParams settings. Provided that a representative training set of galaxy models (such as the COSMOS sample), of sufficient size, is used, the variation in $m_{i \text {,reconv }}$ seen in Fig. 7 should not prevent simulations using the reconvolution algorithm from being accurate to Stage IV requirements for weak lensing.

If greater accuracy is required, users wishing to reduce the impact of these effects can modify the values of the GSP arams according to their needs. In this case, reducing folding_threshold by a factor of 10 brings $m_{2 \text {,reconv within re- }}$ quirements for all 200 galaxies tested. Additive biases $c_{i \text {,reconv }}$ were found to be extremely small (and consistent with zero) in all cases.

These results shows that the approximations inherent in the reconvolution process do not significantly interfere with GALSIM's ability to render accurate images suitable for weak lensing simulations, for a realistic range of galaxy profiles drawn from COSMOS.

\subsection{Limitations}

While results presented in this Section are encouraging, with the default settings providing accuracy that comfortably exceeds our requirements by a factor of 5-10 in many cases, it must be remembered that no set of tests presented in this arti- 
cle could be sufficient to positively validate GALSIM's performance for all possible future applications. Users of GALSIM are strongly advised to conduct their own tests, tailored to their specific requirements.

One specific caveat worthy of mention is the adoption of a circular PSF for all tests presented. A circular Airy was chosen as a simple approximation to the PSF found in COSMOS and other HST images. GALSIM makes no distinction between those objects describing PSFs and those describing galaxies when rendering convolutions of multiple profiles. However, it is possible that a subtle bug or genuine rendering issue might only be activated for cases where both galaxy and PSF break circular symmetry. We stress again that no set of tests performed here can cover all possible uses of the code. Where data properties such as the PSF are known we encourage GALSIM users to perform their own validation and adjust the rendering parameters as required for their particular use case.

Another caveat is that we only used a particular set of COSMOS galaxies for the training sample. It is plausible that galaxy models drawn from a population with a different redshift distribution to the COSMOS sample, or imaged in a filter other than F814W, might have sufficiently different morphological characteristics to fail the rendering requirements adopted in this work. In future it will be of value to understand the marginal, $m_{\sigma \text {,interp }} \simeq 4 \times 10^{-4}$ failure of galaxy size transformations in the RealGalaxy tests when both shear and magnification were applied (see $\$ 9.2$ ).

In many cases, therefore, users may find it necessary to modify and refine the tests presented here, especially where the inputs and requirements of their analyses differ significantly from the assumptions adopted. Some of the tests in this Section will hopefully serve as a useful starting point for these investigations.

\section{Performance}

While the paramount consideration in GALSIM is the accuracy of the renderings, especially with respect to the shapes, we have also paid considerable attention to making the code as fast as possible. Code optimization is of course a wide ranging topic, and we will not attempt to go into detail about all the optimizations included in the GALSIM code. We just mention a few optimizations that were particularly helpful and give some estimates of the timings for typical use cases.

\subsection{Optimizations}

The most important performance decision was to use $\mathrm{C}++$ for all time-critical operations. The Python layer provides a nice front-end user interface, but it is notoriously difficult to do serious coding optimization in Python. So for all significant calculations, GALSIM calls $\mathrm{C}++$ functions from within Python, where we have used the normal kinds of optimization techniques that are standard with $\mathrm{C}$ and $\mathrm{C}++$ code: precomputing values that will be used multiple times, having tight inner loops, using iterators to be more amenable to vectorization by the compiler, using lookup tables and LRU caches, etc.

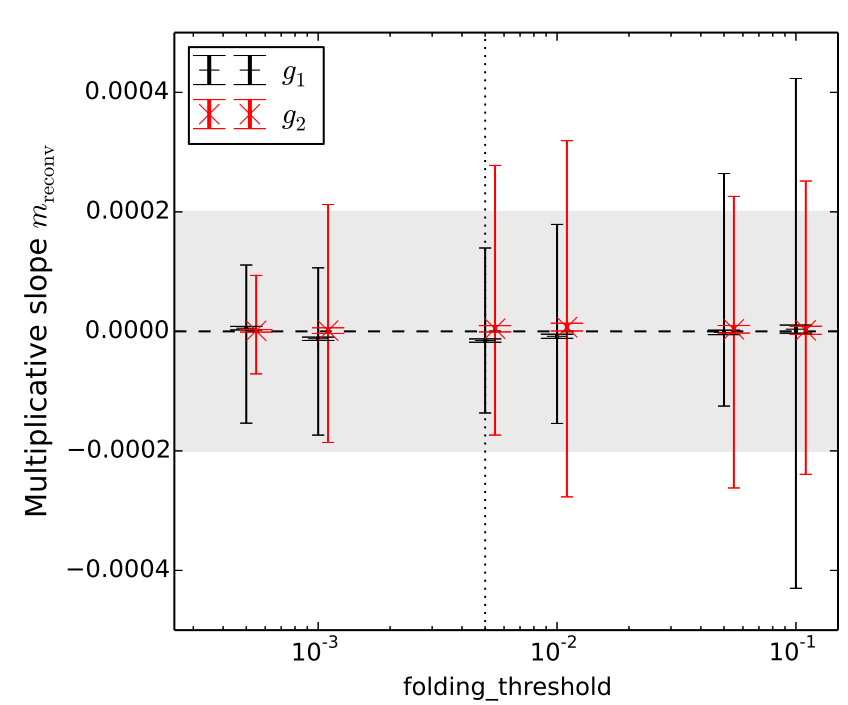

Figure 7: Multiplicative slope $m_{i \text {,reconv }}$ for the reconvolution test of $\S 9.3$, for $g_{1}$ and $g_{2}$, as a function of the folding_threshold parameter (cf. \$6.4). The exterior error bars show the full range of values for the 200 models tested, and the points and interior error bars show the mean and standard error. The shaded region shows the target for GALSIM based on not exceeding one tenth of weak lensing accuracy requirements for Stage IV surveys (see $\$ 9$ ). The default value of folding_threshold is indicated by the dotted line.

The FFTs for computing convolutions in DFT rendering normally constitute the bulk of the calculation time. For these, we use the excellent FFTw package (Frigo \& Johnson, 2005). The calculations are fastest when the image size is either an exact power of 2, or 3 times a power of 2, so GALSIM pads the images to meet this criterion before performing the FFT. Aside from this consideration, we only use as large an FFT image as is required to achieve the desired accuracy. This balance is one of the principal speed/accuracy tradeoffs discussed in $\$ 6.4$.

For some DFT calculations, we can avoid the twodimensional FFT entirely by turning it into a one-dimensional Hankel transform. This is possible when the profile being transformed is radially symmetric, as are many of the analytic profiles. If $f(r, \theta)=f(r)$, then the Fourier transform is similarly radially symmetric:

$$
\begin{aligned}
\widetilde{f}(k) & =\int_{0}^{\infty} r \mathrm{~d} r \int_{0}^{2 \pi} f(r, \theta) e^{\mathrm{i} k r \cos (\theta)} \mathrm{d} \theta \\
& =2 \pi \int_{0}^{\infty} f(r) J_{0}(k r) r \mathrm{~d} r
\end{aligned}
$$

This optimization is particularly effective for Sérsic profiles. The large dynamic range of these profiles, especially for $n>3$, means that a two-dimensional FFT would require a very large image to achieve the appropriate accuracy. It is significantly faster to precompute the one-dimensional Hankel transform $\widetilde{f}(k)$, and use that to fill in the $k$-space values.

The integration package in GALSIM uses an efficient, adaptive Gauss-Kronrod-Patterson algorithm (Patterson, 1968). It starts with the 10-point Gaussian quadrature abscissae and continues adding points at optimally spaced additional locations 
until it either converges or reaches 175 points. If the integral has not converged at that point, it splits the original region in half and tries again on each sub-region, continuing in this manner until all regions have converged. This algorithm is used in GALSIM for Hankel transforms, real-space integration, cumulative probability integrals for photon shooting, calculating the half-light-radius of Sérsic profiles, and a few other places where integrations are required.

Matrix calculations do not generally account for a large share of the running time, but to make these as efficient as possible, we use the TMV library ${ }^{30}$, which calls the system BLAS library when appropriate if such a system library is available (reverting to native code otherwise). The matrix calculations are thus about as efficient as can be expected on a given platform.

One calculation required us to devise our own efficient function evaluation. For interpolated images using the Lanczos interpolant, Fourier-space calculations require evaluation of the Sine integral:

$$
\operatorname{Si}(x)=\int_{0}^{x} \frac{\sin t}{t} \mathrm{~d} t
$$

The standard formulae from Abramowitz \& Stegun (1964) $\$ 5.2$ for computing this efficiently were only accurate to about $10^{-6}$. This was not accurate enough for our needs, and we could not find any other source for a more accurate calculation. We therefore developed our own formulae for efficiently evaluating $\mathrm{Si}(x)$, accurate to $10^{-16}$. The formulae are given in Appendix B.

\subsection{Parallelization}

Another important optimization happens in the Python layer. When running GALSIM using a configuration file, rather than writing Python code, it is very easy to get the code to run using multiple processes using the configuration parameter nproc. Of course, experienced Python programmers can also do this manually, but multiprocessing in Python is not extremely user friendly, so it can be convenient to take advantage of this parallelism already being implemented in GALSIM's configuration file parser.

\subsection{Timings}

The time it takes for GALSIM to render an image depends on many details of the profiles and the image on which they are being rendered. Some profiles tend to take longer than others, and the size of the object relative to the pixel scale of the final image also matters.

For most analytic profiles, if the image scale is not much smaller than the Nyquist scale of the object, rendering using the DFT method will generally take of order 0.01 seconds or less per object. For very many purposes, this kind of profile is perfectly appropriate, and it can include a reasonable amount of complication including multiple components to the galaxy such a bulge plus disk model, offsets of those components, multicomponent PSFs, etc.

Models that include interpolated images take longer. This includes models with an OpticalPSF component or a RealGalaxy. Such profiles typically take of order 0.1 seconds

\footnotetext{
${ }^{30}$ https://code.google.com/p/tmv-cpp/
}

per object for typical sizes of the interpolated images, but the time is highly dependent on the size of the image being used to define the profile.

The running time for photon shooting of course scales linearly with the number of photons. The crossover point where photon shooting is faster than DFT rendering depends on the profile being rendered, but it is typically in the range of $10^{3}-$ $10^{4}$ photons. So for faint objects, it can be significantly faster to use the photon-shooting method.

\subsection{Potential speed pitfalls}

There are a number of potential pitfalls that can lead to slower than normal rendering, which are worth highlighting:

- Whitening. Profiles that use a RealGalaxy can be particularly slow if the resulting image is whitened (cf. §7.5) to remove the correlated noise that is present in the original HST images (and gets exacerbated by the PSF convolution). The whitening step can increase the time per object to about 1 second, although it varies depending on details such as the final pixel scale, what kind of PSF is used, etc.

- Pixel size. Using a DFT to render images with very small pixels relative to the size of the galaxy can be very slow. The time required to perform the FFTs scales as $O\left(N^{2} \log N\right)$ where $N$ is the number of pixels across for the image, so a moderate increase in the size of the galaxy relative to the pixel scale can make a big difference in the running time.

- Sérsic profiles with varying index. Sérsic galaxies require pre-computation of some integrals for each Sérsic index used. When simulating many $n=3.3$ galaxies, the setup time will be amortized over many galaxies and is thus irrelevant. When using a different index $n$ for each galaxy though, the setup must be done for each galaxy, potentially dominating the running time. Thus, it is better to select $n$ from a list of a finite number of values rather than letting it varying continuously within some range.

- Variable optical PSF parameters. The OpticalPSF class similarly has a large setup cost to compute the Fourier transform of the wavefront in the pupil plane. If there is a single PSF for the image, this setup cost is amortized over all the objects, but for a spatially variable PSF (which is more realistic), each object will require a new setup calculation. The variable PSF branches of the GREAT3 challenge (Mandelbaum et al., 2014) were the most time-consuming to generate for this reason.

- Complicated wavelength dependence. Drawing chromatic objects with complex SEDs through non-trivial bandpasses can take quite a long time. The surface brightness profile needs to be integrated over the relevant range of wavelengths. The more complicated the SED and bandpass are, the more samples are required to perform the integration. Thus, depending on the purpose of the images being rendered, it may be advisable to use simple SEDs 
and/or bandpasses, instead of more realistic ones. Optimizations to this process for non-trivial chromatic objects are being incorporated into future versions of GALSIM.

\subsection{Example output}

Figure 8 shows a portion of an image that can be produced by GALSIM. This particular image is from a simulation that is intended to approximate an LSST focal plane.

The simulation has an object density of 70 objects per square arcminute. The galaxies are bulge plus disk models, and the PSF includes both atmospheric seeing and an optical component with the appropriate obscuration for the LSST telescope, struts, and plausible aberrations. $20 \%$ of the objects are stars. The fluxes of both the galaxies and the stars follow a power law distribution, as does the size distribution of the galaxies, and there is an applied cosmological lensing field including both shear and magnification. The noise model includes both Gaussian read noise and Poisson noise on the counts of the detected electrons.

The configuration file to build this image $e^{31}$ is remarkably short (just 122 lines), especially considering how many realistic data features are included. This example showcases why we consider the configuration mode to be one of the strengths of GALSIM. Even for users who are already comfortable writing Python code, the configuration files can be easier to set up and get running in many cases.

Each $4 \mathrm{~K} \times 4 \mathrm{~K}$ chip in this simulation takes about 3 minutes to render on a modern laptop using a single core. The simulation is set to use as many processors as are available, and with 8 cores running at once, the whole field of view (189 chips in all) can be rendered in a bit more than an hour.

Currently GALSIM is unable to simulate many of the features of real images including saturation, bleeding, vignetting, cosmic rays, and the like (cf. §11), so those features are not shown here. For this image, we also chose to cut off the size distribution at 20 arcsec to avoid the rare objects that would require an extremely large FFT.

Despite these apparent shortcomings, this kind of image is still very useful for studying image processing algorithms. Indeed, it is often helpful to intentionally make these and other simplifications to study the behavior of a particular algorithm.

Images similar to the one shown in Figure 8 have been used to study the effects of blending, star-galaxy separation algorithms, and shear estimation. For these and many other investigations, complete realism of the simulated images is not required.

\section{Effects not in GALSIM}

It is worth mentioning some of the many physical effects in optical and near-infrared astronomical observations that GALSIM cannot yet model. Many of these effects will be important to model and explore to help ensure that accurate measurements will be possible from future extragalactic survey data:

\footnotetext{
${ }^{31}$ Available publicly at http://Is.st/xj0.
}

- Physical atmospheric PSF models. Examples of public software that can be used to generate physically motivated, multi-layer phase screen models of atmospheric PSFs include ARROYO ${ }^{32}$, and the LSST IMSIM ${ }^{33}$ software (Peterson et al. in prep.). While the possibility of adding such an atmospheric PSF module to GALSIM was investigated as part of preparatory work for the GREAT3 project (Mandelbaum et al., 2014), this functionality is still lacking.

- Non-linear detector effects. GALSIM cannot yet model pixel saturation or bleeding (e.g. Bertin, 2009), interpixel capacitance (e.g. Moore et al., 2004) and other forms of cross-talk, all of which affect image flux and shape determination.

- Image artifacts such as ghosts, detector persistence. These effects (e.g. Wynne et al., 1984; Meng et al., 2013; Long et al., 2010; Barrick, Ward, \& Cuillandre, 2012; Anderson et al., 2014) can be difficult to model, or even classify and remove, when measuring the properties of astronomical objects in an object-by-object fashion (the current standard procedure ${ }^{34}$ ). Simulating observations with these effects will therefore be important to be able to demonstrate reliable measurements in their presence.

- Cosmic rays. Simulations of these effects (such as, e.g., Rolland et al., 2008) are likely to be an important consideration for imaging in upcoming Stage IV surveys, particularly for space missions at the second Lagrange point (e.g. Barth, Isaacs, \& Poivey, 2000).

- Near field contaminants such as satellite trails and meteors. These effects, of relevance to ground-based imaging, are not currently supported by GALSIM. Like cosmic rays and instrumental artifacts such as ghosts and persistence, these effects will place stringent demands on object classification procedures for upcoming survey experiments.

- Flexion. Although trivially described using a photonshooting approach, simulations of non-affine image transformations such as weak gravitational flexion are incompatible with the Fourier space rendering of individual profiles described in $§ 6$. Flexion is not currently implemented in GALSIM. However, the GALFLEX ${ }^{35}$ package is a standalone, open-source Python module that can be integrated with GALSIM via a NUMPY array interface.

- Vignetting, variable quantum efficiency, and flat fielding effects. No variation in the efficiency of photon detection as a function of position in the output image is currently included in GALSIM, including variation within pixels. It will be possible to address large scale effects in a relatively straightforward post-processing step. However, significant

\footnotetext{
${ }^{32}$ http://cfao.ucolick.org/software/arroyo.php

${ }^{33}$ http: //lsst.astro.washington.edu/

${ }^{34}$ Although see http: //thetractor.org/

35 http://physics.drexel.edu/ jbird/galflex/
} 


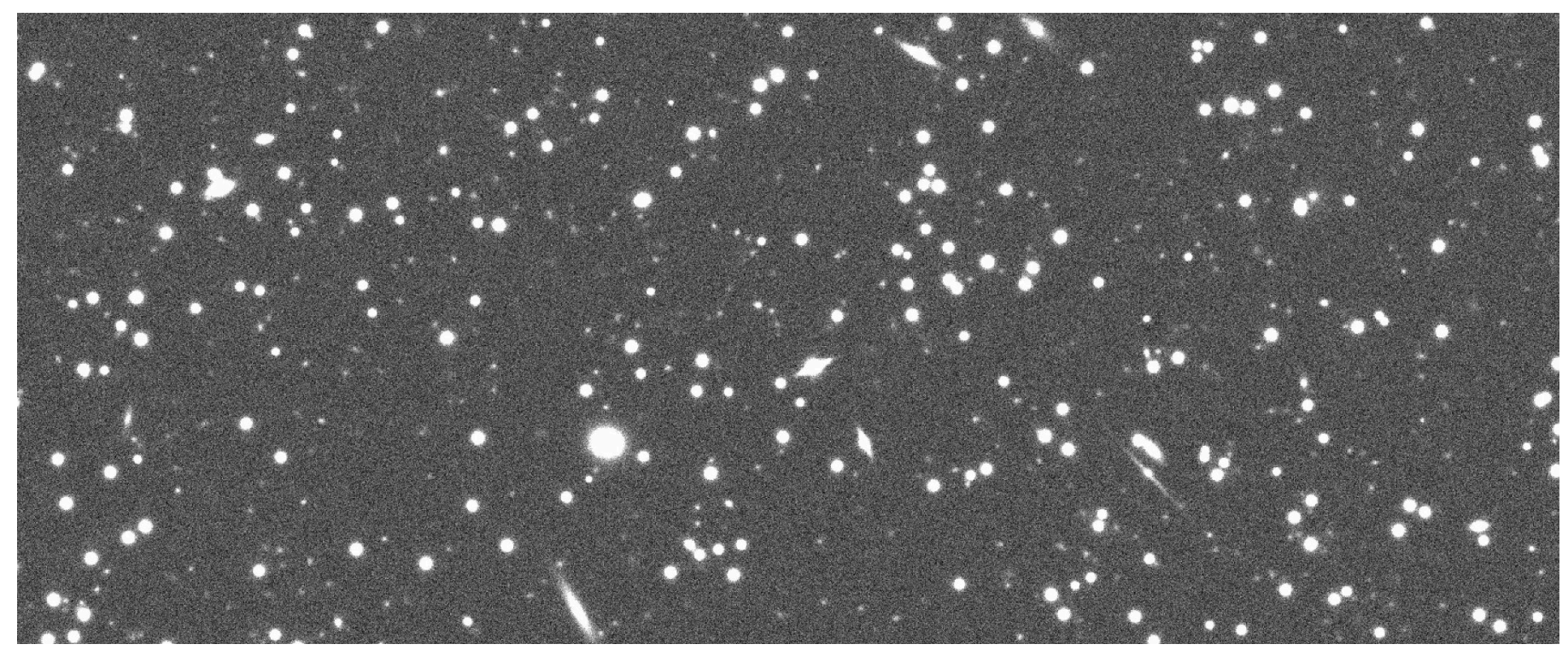

Figure 8: A portion of a GALSIM simulation of an LSST focal plane. This section is about 5 arcminutes across with a number density of 70 objects per square arcmin, of which $80 \%$ are galaxies and $20 \%$ are stars.

intra-pixel quantum efficiency variation may require careful design to render efficiently.

- Complicated WCS functions. Recent studies of the DECam and LSST detectors (Plazas et al., 2014; Rasmussen, 2014) have revealed complicated astrometric shifts, including edge distortions and tree-rings. These are not currently modeled by any existing WCS package, so it would require a custom implementation to include these effects in GALSIM.

Many further possibilities for expanding and improving GALSIM capabilities can be found in already existing functionality. The OpticalPSF class described in $\$ 3.2 .2$ could be extended to a more general treatment of optical wavefronts by allowing an arbitrary number of Zernike coefficients to be specified on input. Support for photon shooting of Shapelet objects (see §3.3.2), although a considerable task, would also be a valuable addition to GALSIM. It is hoped that, through the open and collaborative development pattern already established within the GALSIM project, many of these capabilities will be added to GALSIM in the future.

\section{Conclusions}

We have described GALSIM: the modular galaxy image simulation toolkit. A primary motivation for the project was the desire for an open, transparent, and community-maintained standard toolkit for high precision simulations of the deep extragalactic imaging expected in upcoming Stage III and Stage IV surveys. We have described the range of physical models chosen as the basis for describing astronomical objects in GALSIM, and the support for their description in a range of world coordinate systems. We have also described the mathematical principles used in the multiple strategies supported by GALSIM for rendering these models onto images, and for adding noise.

A number of the techniques employed by GALSIM are novel, including the photon shooting of a range of profiles including InterpolatedImage objects (see §6.3.3) and noise whitening (\$7.5). The consistent handling of astronomical object transformation and rendering under a variety of WCS transformations, described in $\S 5$, is also new in the field of astronomical image simulation. GALSIM is unique in bringing all these features together under a simple, highly flexible, class library interface.

An important part of any software project is testing and validation. The provision of multiple parallel methods for rendering images (see §6), a capability unique to GALSIM among comparable simulation packages, played a vitally important role in this process.

In $\$ 9$ we showed results from a number of tests demonstrating that GALSIM output can describe weak lensing transformations such as shear and magnification at the level of accuracy required for Stage IV surveys such as Euclid, LSST and WFIRSTAFTA. This was demonstrated for highly challenging analytic profiles (the Sersic class, see $\$ 9.1$ ), for surface brightness profiles derived from images such as the InterpolatedImage and RealGalaxy classes (see $\$ 9.2$ ), and for the reconvolution algorithm (see $\$ 9.3$ ).

The accuracy of rendering results can be tuned using input parameters, and using the GSParams structure described in §6.4. Less stringent requirements will yield faster execution, but at the cost of lowering rendering accuracy in output images. As no set of validation tests can completely cover all usage scenarios, the tests of $\$ 9$ should be modified by users of the GALSIM software to tailor them to each application's specific requirements.

As discussed in $\S 11$, there are a number of important effects 
that GALSIM cannot yet simulate, but that may significantly impact data analysis for upcoming surveys. Work is expected to continue in these interesting areas: while the development and support of GALSIM began in early 2012, it is an ongoing project that continues to build on increasing numbers of users (and developers).

As well as being used to generate the images for the GREAT3 challenge (Mandelbaum et al., 2014), GALSIM now provides a considerable open-source toolkit for the simulation of extragalactic survey images. Having been shown to meet demanding tolerances on rendering accuracy in a number of tests, GALSIM simulations can be used as a benchmark for development or common reference point for code comparison. It also provides an accessible, well-documented reference codebase. GALSIM is easily extended, or can be used simply as a class library in the development of further astronomical image analysis applications. In these respects GALSIM achieves its primary aims, and will become an increasingly powerful toolkit for astronomical image simulation following development that is planned for the near future.

\section{Acknowledgments}

We wish to thank the two anonymous referees whose insightful comments significantly improved the paper. This project was supported in part by NASA via the Strategic University Research Partnership (SURP) Program of the Jet Propulsion Laboratory, California Institute of Technology. Part of BR's work was done at the Jet Propulsion Laboratory, California Institute of Technology, under contract with NASA. BR, JZ and TK acknowledge support from a European Research Council Starting Grant with number 240672. MJ acknowledges support from NSF award AST-1138729. RM was supported in part by program HST-AR-12857.01-A, provided by NASA through a grant from the Space Telescope Science Institute, which is operated by the Association of Universities for Research in Astronomy, Incorporated, under NASA contract NAS5-26555; and in part by a Sloan Fellowship. HM acknowledges support from Japan Society for the Promotion of Science (JSPS) Postdoctoral Fellowships for Research Abroad and JSPS Research Fellowships for Young Scientists. PM is supported by the U.S. Department of Energy under Contract No. DE- FG02-91ER40690. The authors acknowledge the use of the UCL Legion High Performance Computing Facility (Legion@UCL), and associated support services, in the completion of this work. This work was supported in part by the National Science Foundation under Grant No. PHYS-1066293 and the hospitality of the Aspen Center for Physics. 


\section{Appendix A. Conventions in the lensing engine}

\section{Appendix A.1. Grid parameters}

All calculations in the GALSIM lensing engine (see §4) approximate real continuous functions using a discrete, finite grid. The real-space grid is defined by

$$
\begin{aligned}
L & =\text { length of grid along one dimension (angular units) } \\
d & =\text { spacing between grid points (angular units) } \\
N & =\text { number of grid points along one dimension }=L / d .
\end{aligned}
$$

There is a comparable grid in Fourier-space (i.e., same $N$ ). Given the parameters of the real-space grid, the $k_{\min }$ along one dimension is $2 \pi / L$, so we can think of this quantity as the grid spacing in Fourier space, i.e., $k_{\min }=\Delta k$. This value of $k_{\min }$ corresponds to a Fourier mode that exactly fits inside of our square grid (in one dimension). The $k$ range, again in one dimension, is from $k_{1}=-\pi / d$ to $\pi / d$, i.e., $\left|k_{1}\right|<k_{\max }=\pi / d$. This corresponds to a mode that is sampled exactly twice (the minimum possible) given our choice of grid spacing. For the two-dimensional grid, the maximum value of $|k|$ is then $\sqrt{2} \pi / d$.

\section{Appendix A.2. Fourier transform properties}

The convention for the Fourier transform defined by equations (34) \& (35) is adopted throughout, as this is the form typically used for describing the statistics of cosmological shear and matter fields. Unlike the convention typically used in computing and signals processing, and in the standard definition of the Discrete Fourier Transform (DFT), this convention is non-unitary and uses an angular (rather than normal) frequency in the exponent ${ }^{36}$. This introduces some additional factors of $2 \pi$ into standard results, and so here we re-express these results using the non-unitary, angular frequency convention.

It can be readily shown that if we define $g(x L) \equiv f(x)$, the following well-known identity holds:

$$
\mathcal{F}\{g(x L)\}=\frac{1}{L} \widetilde{g}\left(\frac{k}{L}\right) .
$$

If we further define $s(h+x) \equiv g(x)$ then it is straightforward to show that

$$
\mathcal{F}\{s(h+x)\}=\mathrm{e}^{\mathrm{i} k h} \widetilde{s}(k) .
$$

There is an additional factor of $2 \pi$ in the exponent when this property of Fourier transforms is expressed using the normal (rather than angular) frequency convention.

The most important result for approximating continuous functions using DFTs is the Poisson summation formula, which under the conventions of equations (34) \& (35) may be stated as

$$
\sum_{n=-\infty}^{\infty} f(n)=\sum_{q=-\infty}^{\infty} \widetilde{f}(2 \pi q)
$$

for integers $n$ and $q$. It should be noted that in the normal frequency, unitary transform convention form of the Poisson summation formula the $2 \pi$ within $\widetilde{f}(2 \pi q)$ is absent. Using equations (A.4) \& (A.5) we arrive at the following useful expression of the Poisson summation formula:

$$
\sum_{n=-\infty}^{\infty} f(n L+x)=\frac{1}{L} \sum_{q=-\infty}^{\infty} \widetilde{f}\left(\frac{2 \pi q}{L}\right) \mathrm{e}^{\mathrm{i} 2 \pi x q / L} .
$$

By substituting $\Delta k \equiv k_{\min }=2 \pi / L$ we write this as

$$
\sum_{n=-\infty}^{\infty} f\left(\frac{2 \pi n}{\Delta k}+x\right)=\left(\frac{\Delta k}{2 \pi}\right) \sum_{q=-\infty}^{\infty} \tilde{f}(q \Delta k) \mathrm{e}^{\mathrm{i} x \Delta k q}
$$

\footnotetext{
${ }^{36}$ For a discussion of transform conventions see http://en.wikipedia.org/wiki/Fourier_transform\#Other_conventions
} 


\section{Appendix A.3. Fourier transform of discrete samples of the power spectrum}

Let us define the following dimensionless function, which takes samples from a power spectrum:

$$
P_{\Delta k}[q, p] \equiv(\Delta k)^{2} P(q \Delta k, p \Delta k)
$$

We will use square brackets to denote functions with discrete, integer input variables, as here. We will also use $n, m$ for integer indices in real space summations, and $q, p$ in Fourier space. It can then be shown that

$$
\mathcal{F}^{-1}\left\{\sum_{q, p=-\infty}^{\infty} \delta\left(k_{1}-q \Delta k\right) \delta\left(k_{2}-p \Delta k\right) P_{\Delta k}[q, p]\right\}=\sum_{n, m=-\infty}^{\infty} \xi_{+}\left(\theta_{1}-\frac{2 \pi n}{\Delta k}, \theta_{2}-\frac{2 \pi m}{\Delta k}\right),
$$

where we have used the Fourier series expression for the Dirac comb function, and the convolution theorem. We note that this expression is still continuous, but describes an infinite, periodic summation (of period $L=2 \pi / \Delta k$ ) of copies of the correlation function $\xi_{+}$. For sufficiently small $\Delta k$, these copies may be spaced sufficiently widely in the real domain to be able to learn much about $\xi_{+}\left(\theta_{1}, \theta_{2}\right)$ in the non-overlapping regions. We therefore define this function as

$$
\xi_{\frac{2 \pi}{\Delta k}}\left(\theta_{1}, \theta_{2}\right) \equiv \sum_{n=-\infty}^{\infty} \xi_{+}\left(\theta_{1}-\frac{2 \pi n}{\Delta k}, \theta_{2}-\frac{2 \pi m}{\Delta k}\right)
$$

Using the expression of the Poisson summation formula in equation (A.8), we can also write

$$
\xi_{\frac{2 \pi}{\Delta k}}\left(\theta_{1}, \theta_{2}\right)=\sum_{q, p=-\infty}^{\infty}\left(\frac{\Delta k}{2 \pi}\right)^{2} P(q \Delta k, p \Delta k) \mathrm{e}^{\mathrm{i} \Delta k\left(\theta_{1} q+\theta_{2} p\right)}=\frac{1}{(2 \pi)^{2}} \sum_{q, p=-\infty}^{\infty} P_{\Delta k}[q, p] \mathrm{e}^{\mathrm{i} \Delta k\left(\theta_{1} q+\theta_{2} p\right)},
$$

This is in fact an expression for the inverse of the Discrete Time Fourier Transform (DTFT), although this result is normally derived using unitary conventions for the transform pair.

The expression in equation (A.12) is periodic with period $2 \pi / \Delta k=L$. All of the information it contains about $\xi_{+}\left(\theta_{1}, \theta_{2}\right)$ is therefore also contained in one period of the function only. For approximating this information discretely, as desired in numerical analysis, we can imagine taking $N$ equally spaced samples of the function in equation (A.12) along a single period $L$ in each dimension. These samples are therefore separated by $\Delta \theta=2 \pi / \Delta k N=d$ in real space, and we define the sampled function itself as

$$
\xi_{\Delta \theta}[n, m] \equiv \xi_{\frac{2 \pi}{\Delta k}}(n \Delta \theta, m \Delta \theta)=\frac{1}{(2 \pi)^{2}} \sum_{q, p=-\infty}^{\infty} P_{\Delta k}[q, p] \mathrm{e}^{\mathrm{i} 2 \pi(q n+p m) / N}
$$

for the integer indices $n, m=0,1, \ldots, N-1$, by substitution into equation (A.12). Using the periodicity of the exponential term in the expression above, this may be written as

$$
\xi_{\Delta \theta}[n, m]=\frac{1}{(2 \pi)^{2}} \sum_{q, p=0}^{N-1} P_{N}[q, p] \mathrm{e}^{\mathrm{i} 2 \pi(q n+p m) / N}
$$

where we have defined

$$
P_{N}[q, p] \equiv \sum_{i, j=-\infty}^{\infty} P_{\Delta k}[q-i N, p-j N] .
$$

Needless to say, in order to be able to calculate the values of $P_{N}[q, p]$ in practice we must also truncate the $P_{\Delta k}$ sequence to be finite in length. A very common choice is to use the same number $N$ of samples in both real and Fourier space. Choosing to use only $N$ samples from $P_{\Delta k}$ then gives

$$
\begin{aligned}
\xi_{\Delta \theta}[n, m] & =\frac{1}{(2 \pi)^{2}} \sum_{q, p=0}^{N-1} P_{\Delta k}[q, p] \mathrm{e}^{\mathrm{i} 2 \pi(q n+p m) / N} \\
& =\frac{1}{N^{2}(\Delta \theta)^{2}} \sum_{q, p=0}^{N-1} P(q \Delta k, p \Delta k) \mathrm{e}^{\mathrm{i} 2 \pi(q n+p m) / N}
\end{aligned}
$$

For greater detail regarding this calculation, please see the lensing engine document in the GALSIM repository. ${ }^{37}$

\footnotetext{
${ }^{37}$ https://github.com/GalSim-developers/Galsim/blob/master/devel/modules/lensing_engine.pdf
} 
The relationship in equation (A.16) is in fact the inverse Discrete Fourier Transform for our non-unitary convention. The more usual definition of the inverse DFT, and that adopted by many DFT implementations (including the NUMPY package in Python), is

$$
f[n, m]=\text { numpy } \cdot \text { fft. } \operatorname{ifft2}(\widetilde{f}[p, q]) \equiv \frac{1}{N^{2}} \sum_{q, p=0}^{N-1} \widetilde{f}[q, p] \mathrm{e}^{\mathrm{i} 2 \pi(q n+p m) / N} \quad \text { (NuMPY convention) }
$$

(The factor of $1 / N^{2}$ here is often found in conventions for the inverse DFT, and ensures that the DFT followed by the inverse DFT yields the original, input array.) The Fast Fourier Transform algorithm allows the DFT to be calculated very efficiently.

\section{Appendix A.4. Generating Gaussian fields}

Using equation (A.16) we see how to construct a realization of a Gaussian random lensing field on a grid, with an ensemble average power spectrum that is approximately $P(k)$. We create the following complex, 2D array of dimension $N$ in Fourier space:

$$
\widetilde{\kappa}[p, q]=\widetilde{\kappa}_{E}[p, q]+\widetilde{\mathrm{i}}_{B}[p, q],
$$

where $\widetilde{\kappa}_{E}$ is defined in terms of the desired E-mode power spectrum $P_{E}$ as

$$
\widetilde{\kappa}_{E}[p, q]=\frac{1}{N \Delta \theta} \sqrt{\frac{P_{E}(p \Delta k, q \Delta k)}{2}}\{\mathcal{N}(0,1)+\mathrm{i} \mathcal{N}(0,1)\},
$$

and likewise for $\widetilde{\kappa}_{B}$. Here $\mathcal{N}(0,1)$ denotes a standard Gaussian random deviate drawn independently at each $[p, q]$ array location. This complex-valued Fourier-space convergence can be used to construct the Fourier-space shear field via ${ }^{38}$

$$
\widetilde{g}=\mathrm{e}^{2 \mathrm{i} \psi} \widetilde{\kappa}
$$

where $\mathrm{e}^{2 \mathrm{i} \psi}$ is the phase of the $k$ vector defined as $k=\Delta k(p+\mathrm{i} q)$. It can be seen that $\left.\widehat{g}[p, q] \widetilde{g}^{*}\left[p^{\prime}, q^{\prime}\right]\right\rangle=\delta_{p}^{p^{\prime}} \delta_{q}^{q^{\prime}} P(|k|) /(N \Delta \theta)^{2}$ where $|k|=\Delta k \sqrt{p^{2}+q^{2}}$. Taking the inverse DFT of $\widetilde{g}$ provides the realization of the Gaussian lensing field $g[n, m]$ on a grid in real space, where the real (imaginary) parts of $g[n, m]$ correspond to the first (second) shear components. From equation (A.16), $g[n, m]$ will be correctly scaled to have discrete correlation function $\xi_{\Delta \theta}[n, m]$ by construction.

\section{Appendix B. Efficient evaluation of the Sine and Cosine integrals}

The trigonometric integrals are defined as

$$
\begin{aligned}
& \operatorname{Si}(x)=\int_{0}^{x} \frac{\sin t}{t} \mathrm{~d} t=\frac{\pi}{2}-\int_{x}^{\infty} \frac{\sin t}{t} \mathrm{~d} t \\
& \operatorname{Ci}(x)=\gamma+\ln x+\int_{0}^{x} \frac{\cos t-1}{t} \mathrm{~d} t=-\int_{x}^{\infty} \frac{\cos t}{t} \mathrm{~d} t
\end{aligned}
$$

where $\gamma$ is the Euler-Mascheroni constant. In GALSIM, calculations involving Lanczos interpolants require an efficient calculation of $\operatorname{Si}(x)$. As we were unable to find a source for an efficient calculation that was suitably accurate, we developed our own, which we present here. We do not use $\operatorname{Ci}(x)$ anywhere in the code, but for completeness, we provide a similarly efficient and accurate calculation of it as well.

For small arguments, we use Padé approximants of the convergent Taylor series

$$
\begin{aligned}
\operatorname{Si}(x) & =\sum_{n=0}^{\infty} \frac{(-1)^{n} x^{2 n+1}}{(2 n+1)(2 n+1) !} \\
\operatorname{Ci}(x) & =\gamma+\ln x+\sum_{n=1}^{\infty} \frac{(-1)^{n} x^{2 n}}{2 n(2 n) !}
\end{aligned}
$$

\footnotetext{
${ }^{38}$ See, for example, Kaiser \& Squires (1993), but note that there is a sign error in equation 2.1.12 in that paper that is corrected in equation 2.1.15.
} 
Using the Maple software package ${ }^{39}$, we derived the following formulae, which are accurate to better than $10^{-16}$ for $0 \leq x \leq 4$ :

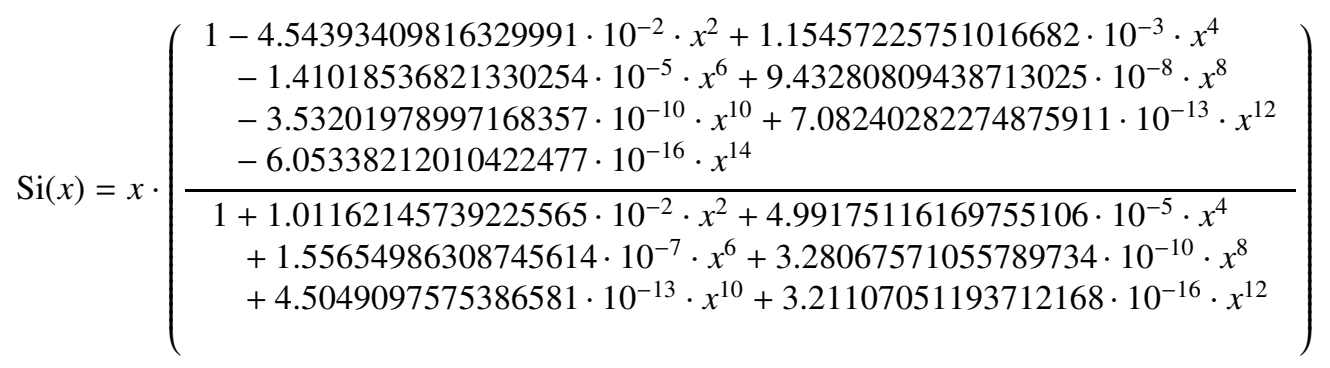

$\operatorname{Ci}(x)=\gamma+\ln (x)+$

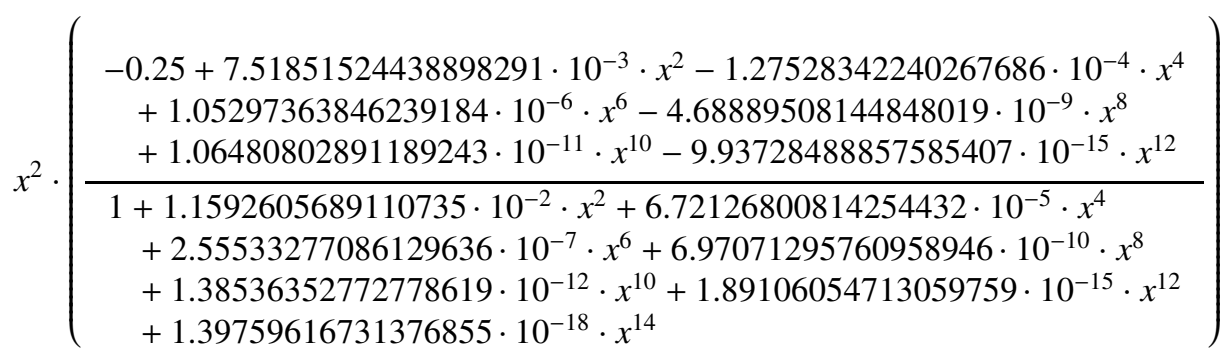

For $x>4$, one can use the helper functions:

$$
\begin{aligned}
& f(x) \equiv \int_{0}^{\infty} \frac{\sin (t)}{t+x} \mathrm{~d} t=\int_{0}^{\infty} \frac{e^{-x t}}{t^{2}+1} \mathrm{~d} t=\mathrm{Ci}(x) \sin (x)+\left(\frac{\pi}{2}-\operatorname{Si}(x)\right) \cos (x) \\
& g(x) \equiv \int_{0}^{\infty} \frac{\cos (t)}{t+x} \mathrm{~d} t=\int_{0}^{\infty} \frac{t e^{-x t}}{t^{2}+1} \mathrm{~d} t=-\operatorname{Ci}(x) \cos (x)+\left(\frac{\pi}{2}-\operatorname{Si}(x)\right) \sin (x)
\end{aligned}
$$

using which, the trigonometric integrals may be expressed as

$$
\begin{aligned}
\operatorname{Si}(x) & =\frac{\pi}{2}-f(x) \cos (x)-g(x) \sin (x) \\
\mathrm{Ci}(x) & =f(x) \sin (x)-g(x) \cos (x)
\end{aligned}
$$

In the limit as $x \rightarrow \infty, f(x) \rightarrow 1 / x$ and $g(x) \rightarrow 1 / x^{2}$. Thus, we can use Chebyshev-Padé expansions of $\frac{1}{\sqrt{y}} f\left(\frac{1}{\sqrt{y}}\right)$ and $\frac{1}{y} g\left(\frac{1}{\sqrt{y}}\right)$ in the interval $0 . . \frac{1}{4^{2}}$ to obtain the following approximants, good to better than $10^{-16}$ for $x \geq 4$ :

$$
\begin{aligned}
& f(x)=\frac{1}{x} \cdot\left(\begin{array}{rl}
1 & +7.44437068161936700618 \cdot 10^{2} \cdot x^{-2}+1.96396372895146869801 \cdot 10^{5} \cdot x^{-4} \\
& +2.37750310125431834034 \cdot 10^{7} \cdot x^{-6}+1.43073403821274636888 \cdot 10^{9} \cdot x^{-8} \\
& +4.33736238870432522765 \cdot 10^{10} \cdot x^{-10}+6.40533830574022022911 \cdot 10^{11} \cdot x^{-12} \\
& +4.20968180571076940208 \cdot 10^{12} \cdot x^{-14}+1.00795182980368574617 \cdot 10^{13} \cdot x^{-16} \\
& +4.94816688199951963482 \cdot 10^{12} \cdot x^{-18}-4.94701168645415959931 \cdot 10^{11} \cdot x^{-20} \\
\hline 1+ & 7.46437068161927678031 \cdot 10^{2} \cdot x^{-2}+1.97865247031583951450 \cdot 10^{5} \cdot x^{-4} \\
& +2.41535670165126845144 \cdot 10^{7} \cdot x^{-6}+1.47478952192985464958 \cdot 10^{9} \cdot x^{-8} \\
& +4.58595115847765779830 \cdot 10^{10} \cdot x^{-10}+7.08501308149515401563 \cdot 10^{11} \cdot x^{-12} \\
& +5.06084464593475076774 \cdot 10^{12} \cdot x^{-14}+1.43468549171581016479 \cdot 10^{13} \cdot x^{-16}
\end{array}\right) \\
& g(x)=\frac{1}{x^{2}} \cdot\left(\begin{array}{rl}
1 & +8.1359520115168615 \cdot 10^{2} \cdot x^{-2}+2.35239181626478200 \cdot 10^{5} \cdot x^{-4} \\
& +3.12557570795778731 \cdot 10^{7} \cdot x^{-6}+2.06297595146763354 \cdot 10^{9} \cdot x^{-8} \\
& +6.83052205423625007 \cdot 10^{10} \cdot x^{-10}+1.09049528450362786 \cdot 10^{12} \cdot x^{-12} \\
& +7.57664583257834349 \cdot 10^{12} \cdot x^{-14}+1.81004487464664575 \cdot 10^{13} \cdot x^{-16} \\
& +6.43291613143049485 \cdot 10^{12} \cdot x^{-18}-1.36517137670871689 \cdot 10^{12} \cdot x^{-20} \\
\hline 1+ & 8.19595201151451564 \cdot 10^{2} \cdot x^{-2}+2.40036752835578777 \cdot 10^{5} \cdot x^{-4} \\
& +3.26026661647090822 \cdot 10^{7} \cdot x^{-6}+2.23355543278099360 \cdot 10^{9} \cdot x^{-8} \\
& +7.87465017341829930 \cdot 10^{10} \cdot x^{-10}+1.39866710696414565 \cdot 10^{12} \cdot x^{-12} \\
& +1.17164723371736605 \cdot 10^{13} \cdot x^{-14}+4.01839087307656620 \cdot 10^{13} \cdot x^{-16} \\
& +3.99653257887490811 \cdot 10^{13} \cdot x^{-18}
\end{array}\right)
\end{aligned}
$$

\footnotetext{
${ }^{39}$ http: //www.maplesoft.com/
} 
Thus we can calculate $\operatorname{Si}(x)$ to double precision accuracy for any value of $x$. In GALSIM, all of the above polynomials are implemented using Horner's rule, so they are very efficient to evaluate.

Abazajian, K. N., Adelman-McCarthy, J. K., Agüeros, M. A., et al. 2009, ApJS, 182, 543

Abramowitz, M., \& Stegun, I. 1964, Handbook of Mathematical Functions, 5th edn. (New York: Dover)

Airy, G. B. 1835, Transactions of the Cambridge Philosophical Society, 5, 283

Albrecht, A., Bernstein, G., Cahn, R., et al. 2006, ArXiv Astrophysics e-prints, astro-ph/0609591

Amara, A., \& Réfrégier, A. 2008, MNRAS, 391, 228

Anderson, R. E., Regan, M., Valenti, J., \& Bergeron, E. 2014, ArXiv e-prints, arXiv:1402.4181

Antilogus, P., Astier, P., Doherty, P., Guyonnet, A., \& Regnault, N. 2014, Journal of Instrumentation, 9, C3048

Bacon, D. J., Goldberg, D. M., Rowe, B. T. P., \& Taylor, A. N. 2006, MNRAS, 365, 414

Barrick, G. A., Ward, J., \& Cuillandre, J.-C. 2012, in Society of Photo-Optical Instrumentation Engineers (SPIE) Conference Series, Vol. 8453, High Energy,

Optical, and Infrared Detectors for Astronomy V, article id. $84531 \mathrm{~K}, 8 \mathrm{pp}$.

Bartelmann, M. 1996, A\&A, 313, 697

-. 2010, Classical and Quantum Gravity, 27, 233001

Barth, J., Isaacs, J., \& Poivey, C. 2000, The Radiation Environment for the Next Generation Space Telescope, Tech. rep.

Becker, M. R. 2013, MNRAS, 435, 115

Bernstein, G. M. 2010, MNRAS, 406, 2793

Bernstein, G. M., \& Armstrong, R. 2014, MNRAS, 438, 1880

Bernstein, G. M., \& Gruen, D. 2014, PASP, 126, 287

Bernstein, G. M., \& Jarvis, M. 2002, AJ, 123, 583

Bertin, E. 2009, Mem. Societa Astronomica Italiana, 80, 422

Born, M., \& Wolf, E. 1999, Principles of Optics

Bridle, S., Shawe-Taylor, J., Amara, A., et al. 2009, Annals of Applied Statistics, 3, 6

Bridle, S., Balan, S. T., Bethge, M., et al. 2010, MNRAS, 405, 2044

Calabretta, M. R., \& Greisen, E. W. 2002, A\&A, 395, 1077

Casertano, S., de Mello, D., Dickinson, M., et al. 2000, AJ, 120, 2747

Ciotti, L., \& Bertin, G. 1999, A\&A, 352, 447

Cropper, M., Hoekstra, H., Kitching, T., et al. 2013, MNRAS, 431, 3103

Cypriano, E. S., Amara, A., Voigt, L. M., et al. 2010, MNRAS, 405, 494

de Jong, J. T. A., Verdoes Kleijn, G. A., Kuijken, K. H., \& Valentijn, E. A. 2013, Experimental Astronomy, 35, 25

de Vaucouleurs, G. 1948, Annales d'Astrophysique, 11, 247

-. 1959, AJ, 64, 397

Duncan, C. A. J., Joachimi, B., Heavens, A. F., Heymans, C., \& Hildebrandt, H. 2014, MNRAS, 437, 2471

Fried, D. L. 1966, Journal of the Optical Society of America (1917-1983), 56, 1372

Frigo, M., \& Johnson, S. G. 2005, Proceedings of the IEEE, 93, 216, special issue on "Program Generation, Optimization, and Platform Adaptation"

Fruchter, A. S., \& Hook, R. N. 2002, PASP, 114, 144

Goldberg, D. M., \& Bacon, D. J. 2005, ApJ, 619, 741

Heymans, C., Van Waerbeke, L., Bacon, D., et al. 2006, MNRAS, 368, 1323

Heymans, C., Van Waerbeke, L., Miller, L., et al. 2012, MNRAS, 427, 146

Hirata, C., \& Seljak, U. 2003, MNRAS, 343, 459

Hirata, C. M., Mandelbaum, R., Seljak, U., et al. 2004, MNRAS, 353, 529

Hogg, D. W., \& Lang, D. 2013, PASP, 125, 719

Huterer, D. 2010, General Relativity and Gravitation, 42, 2177

Huterer, D., Takada, M., Bernstein, G., \& Jain, B. 2006, MNRAS, 366, 101

Irwin, J., \& Shmakova, M. 2005, New Astron. Rev., 49, 83

Johnson, N., Kemp, A., \& Kotz, S. 2005, Univariate Discrete Distributions, Wiley Series in Probability and Statistics (Hoboken, New Jersey: John Wiley \& Sons)

Kacprzak, T., Bridle, S., Rowe, B., et al. 2014, MNRAS, 441, 2528

Kacprzak, T., Zuntz, J., Rowe, B., et al. 2012, MNRAS, 427, 2711

Kaiser, N. 2000, ApJ, 537, 555

Kaiser, N., \& Squires, G. 1993, ApJ, 404, 441

Kaiser, N., Squires, G., \& Broadhurst, T. 1995, ApJ, 449, 460

Kitching, T. D., Balan, S. T., Bridle, S., et al. 2012, MNRAS, 423, 3163

Kitching, T. D., Rowe, B., Gill, M., et al. 2013, ApJS, 205, 12

Koekemoer, A. M., Fruchter, A. S., Hook, R. N., \& Hack, W. 2003, in HST Calibration Workshop : Hubble after the Installation of the ACS and the NICMOS Cooling System, ed. S. Arribas, A. Koekemoer, \& B. Whitmore, 337

Koekemoer, A. M., Aussel, H., Calzetti, D., et al. 2007, ApJS, 172, 196

Lackner, C. N., \& Gunn, J. E. 2012, MNRAS, 421, 2277

Lauer, T. R. 1999, PASP, 111, 227

Laureijs, R., Amiaux, J., Arduini, S., et al. 2011, ArXiv e-prints, arXiv:1110.3193

Leauthaud, A., Massey, R., Kneib, J.-P., et al. 2007, ApJS, 172, 219

Long, K. S., Bagett, S. M., Deustua, S., \& Riess, A. 2010, in 2010 Space Telescope Science Institute Calibration Workshop - Hubble after SM4. Preparing JWST, held 21-23 July 2010 at Space Telescope Science Institute. Edited by Susana Deustua and Cristina Oliveira. Published online at http://www.stsci.edu/institute/conference/cal10/proceedings, p.26

Luppino, G. A., \& Kaiser, N. 1997, ApJ, 475, 20

Makinson, D. 2012, Sets, Logic and Maths for Computing, Undergraduate Topics in Computer Science (London: Springer-Verlag)

Mandelbaum, R., Hirata, C. M., Leauthaud, A., Massey, R. J., \& Rhodes, J. 2012, MNRAS, 420, 1518

Mandelbaum, R., Hirata, C. M., Seljak, U., et al. 2005, MNRAS, 361, 1287

Mandelbaum, R., Rowe, B., Bosch, J., et al. 2014, ApJS, 212, 5

Massey, R., \& Refregier, A. 2005, MNRAS, 363, 197

Massey, R., Stoughton, C., Leauthaud, A., et al. 2010, MNRAS, 401, 371

Massey, R., Heymans, C., Bergé, J., et al. 2007, MNRAS, 376, 13 
Massey, R., Hoekstra, H., Kitching, T., et al. 2013, MNRAS, 429, 661

Melchior, P., Böhnert, A., Lombardi, M., \& Bartelmann, M. 2010, A\&A, 510, A75

Melchior, P., \& Viola, M. 2012, MNRAS, 424, 2757

Meng, Z., Zhou, X., Zhang, H., et al. 2013, PASP, 125, 1015

Meyers, J. E., \& Burchat, P. R. 2014, Journal of Instrumentation, 9, C3037

Miyazaki, S., Komiyama, Y., Nakaya, H., et al. 2012, in Society of Photo-Optical Instrumentation Engineers (SPIE) Conference Series, Vol. 8446, Society of Photo-Optical Instrumentation Engineers (SPIE) Conference Series

Moffat, A. F. J. 1969, A\&A, 3, 455

Moore, A. C., Ninkov, Z., \& Forrest, W. J. 2004, in Society of Photo-Optical Instrumentation Engineers (SPIE) Conference Series, Vol. 5167, Focal Plane Arrays

for Space Telescopes, ed. T. J. Grycewicz \& C. R. McCreight, 204-215

Nakajima, R., \& Bernstein, G. 2007, AJ, 133, 1763

Navarro, J. F., Frenk, C. S., \& White, S. D. M. 1996, ApJ, 462, 563

Noll, R. J. 1976, Journal of the Optical Society of America (1917-1983), 66, 207

Patterson, F. S. 1940, Harvard College Observatory Bulletin, 914, 9

Patterson, T. 1968, Mathematics of Computation, 22, 847

Peacock, J. A., Schneider, P., Efstathiou, G., et al. 2006, ESA-ESO Working Group on "Fundamental Cosmology", Tech. rep., arXiv:0610906

Pence, W. D., Chiappetti, L., Page, C. G., Shaw, R. A., \& Stobie, E. 2010, A\&A, 524, A42

Plazas, A. A., \& Bernstein, G. 2012, PASP, 124, 1113

Plazas, A. A., Bernstein, G. M., \& Sheldon, E. S. 2014, Journal of Instrumentation, 9, C4001

Press, W. H., Teukolsky, S. A., Vetterling, W. T., \& Flannery, B. P. 1992, Numerical recipes in C: The art of scientific computing, 2nd ed. (Cambridge: Cambridge University Press)

Racine, R. 1996, PASP, 108, 699

Rasmussen, A. 2014, Journal of Instrumentation, 9, C4027

Refregier, A. 2003, MNRAS, 338, 35

Refregier, A., Kacprzak, T., Amara, A., Bridle, S., \& Rowe, B. 2012, MNRAS, 425, 1951

Reyes, R., Mandelbaum, R., Gunn, J. E., et al. 2012, MNRAS, 425, 2610

Rhodes, J., Leauthaud, A., Stoughton, C., et al. 2010, PASP, 122, 439

Rolland, G., Pinheiro da Silva, L., Inguimbert, C., et al. 2008, Nuclear Science, IEEE Transactions on, 55, 2070

Rowe, B., Bacon, D., Massey, R., et al. 2013, MNRAS, 435, 822

Sánchez, E., et al. 2010, Journal of Physics Conference Series, 259, 012080

Schmidt, F., Leauthaud, A., Massey, R., et al. 2012, ApJL, 744, L22

Schneider, P. 2006, in Saas-Fee Advanced Course 33: Gravitational Lensing: Strong, Weak and Micro, ed. G. Meylan, P. Jetzer, P. North, P. Schneider, C. S.

Kochanek, \& J. Wambsganss (Berlin: Springer), 269

Schneider, P., van Waerbeke, L., \& Mellier, Y. 2002, A\&A, 389, 729

Scoville, N., Aussel, H., Brusa, M., et al. 2007, ApJS, 172, 1

Semboloni, E., Hoekstra, H., Huang, Z., et al. 2013, MNRAS, 432, 2385

Sérsic, J. L. 1963, Boletin de la Asociacion Argentina de Astronomia La Plata Argentina, 6, 41

Seshadri, S., Shapiro, C., Goodsall, T., et al. 2013, PASP, 125, 1065

Sheldon, E. S. 2014, MNRAS, 444, L25

van Waerbeke, L. 2010, MNRAS, 401, 2093

Velander, M., Kuijken, K., \& Schrabback, T. 2011, MNRAS, 412, 2665

Voigt, L. M., \& Bridle, S. L. 2010, MNRAS, 404, 458

Voigt, L. M., Bridle, S. L., Amara, A., et al. 2012, MNRAS, 421, 1385

Wright, C. O., \& Brainerd, T. G. 2000, ApJ, 534, 34

Wynne, C. G., Worswick, S. P., Lowne, C. M., \& Jorden, P. R. 1984, The Observatory, 104, 23

Zernike, F. 1934, MNRAS, 94, 377 